\title{
Review
}

\section{Solvation of ions in Helium}

\author{
Tomás González-Lezana ${ }^{\mathrm{a} \dagger}$, Olof Echt ${ }^{\mathrm{b}, \mathrm{c} *}$, Michael Gatchell ${ }^{\mathrm{b}, \mathrm{d}}$, Massimiliano \\ Bartolomei $^{\mathrm{a}}$, José Campos-Martínez ${ }^{\mathrm{a}}$ and Paul Scheier ${ }^{\mathrm{b} \ddagger}$ \\ ${ }^{a}$ Instituto de Física Fundamental, Consejo Superior de Investigaciones Científicas \\ IFF-CSIC, Serrano 123, 28006 Madrid, Spain; \\ ${ }^{\mathrm{b}}$ Institut für Ionenphysik und Angewandte Physik, Universität Innsbruck, Technikerstr. \\ 25, A-6020 Innsbruck, Austria; \\ ${ }^{\mathrm{c}}$ Department of Physics, University of New Hampshire, Durham, New Hampshire 03824, \\ United States; \\ d Department of Physics, Stockholm University, 10691 Stockholm, Sweden
}

(May 2020)

\begin{abstract}
In recent years several reviews of ${ }^{4} \mathrm{He}$ nanodroplets (HNDs) have been published in this Journal. The present one focuses on the solvation of atomic, molecular or cluster ions $\mathrm{X}^{ \pm}$ in HNDs. After briefly reviewing the properties of so-called snowballs in bulk helium we discuss experimental conditions for the efficient synthesis of charged, doped HNDs. We show that the cluster ions observed in conventional mass spectrometers originate from fission of highly charged HNDs. Mass spectra of alkali clusters recorded near the ionization threshold of HNDs reveal the size limit beyond which the neutral cluster will be fully embedded in the HND. The abundance distributions of $\mathrm{He}_{N} \mathrm{X}^{ \pm}$ions solvated in helium frequently reveal local anomalies or magic numbers. We demonstrate that the ion abundance is approximately proportional to the dissociation energy $D_{N}=E_{N}-E_{N-1}$. A compilation of observed magic numbers will be presented together with theoretical data, including data for ions solvated in molecular hydrogen. Alternative methods to forming $\mathrm{He}_{N} \mathrm{X}^{+}$that do not rely on nozzle expansions will be summarized. Electronic excitation spectra of $\mathrm{C}_{60}^{+}$and polycyclic aromatic hydrocarbon ions with up to 100 adsorbed helium atoms reveal the properties of the helium adsorption layer in quantitative detail. Next we discuss theoretical efforts to describe the interaction between ions and helium. We close with summarizing the size dependence of physical quantities computed for atomic alkali and alkaline earth cations in helium, such as binding energy, superfluid fraction, structural order, radial density profiles, and the existence of first and higher solvation shells.
\end{abstract}

\section{Contents}

\section{Introduction}

2. Snowballs and bubbles

2.1 Early work on ions in liquid bulk helium

2.2 Early beam studies of pure and doped helium droplets

3. Experimental conditions for the efficient synthesis of snowballs and ionization of helium nanodroplets

3.1 Formation of helium nanodroplets

3.2 Ionization of helium nanodroplets

$\dagger$ Email: t.gonzalez.lezana@csic.es * olof.echt@unh.edu ${ }^{\ddagger}$ paul.scheier@uibk.ac.at

ISSN: print/ISSN online (C) 2020 Taylor \& Francis DOI: http://www.tandfonline.com 
3.3 Mass spectra upon ionization of pure and doped helium nanodroplets

3.4 Large charged helium nanodroplets

3.5 Multiply-charged helium nanodroplets

3.6 Efficiency of snowball formation

4. Impurity location

5. Cluster size distributions

5.1 The relation between abundance and stability of solvated ions 17

5.2 The sizes of particularly stable $\mathrm{He}_{N} \mathrm{X}^{ \pm}$and $\left(\mathrm{H}_{2}\right)_{N} \mathrm{X}^{ \pm} \quad 21$

5.3 Ion molecule reactions, fragmentation, anions, multiply charged ions $\quad 27$

6. Other experimental work 30

7. Applications in spectroscopy 32

8. The theoretical description $\quad 35$

8.1 Interactions $\quad 35$

8.2 Structure and stability 39

9. Conclusions 42

10. Acknowledgments 43

\section{Introduction}

The first successful production of helium clusters in nozzle beams was achieved by Becker and coworkers at the Nuclear Research Center Karlsruhe [1]. The research was a byproduct of efforts to synthesize large deuterium and tritium clusters which were promising candidates for the efficient, directed introduction of fuel into a thermonuclear fusion plasma [2]. Thus, the first nano-sized droplets reportedly produced in nozzle beams were composed of helium, hydrogen, or nitrogen.

While interest in the use of hydrogen clusters for fusion plasmas faded, nozzle sources and gas aggregation sources were successfully refined to produce clusters of pretty much any element, and many molecular materials. Contrary to helium clusters, some of these novel systems showed promise for real-world applications which stimulated a burst of activities in cluster research in the 1980ies. Until 1990, research by Gspann and coworkers at Karlsruhe in which HNDs were scattered off electrons, atoms and surfaces [3-8] was the only persistent research program involving He clusters. It was evident, though, that these systems presented an intriguing opportunity for experimentalists and theorists alike to understand the effects of quantum statistics when finite size effects play a prominent role [9-11]. Excellent reviews of early work have been published by Whaley, Becker and Northby [11-13].

Around 1990, Scoles' group at Princeton and Toennies' group at Göttingen realized that HNDs could be readily doped with atoms or molecules, and that those doped HNDs provide excellent samples for high resolution spectroscopy of ultracold molecules [14-17]. Very soon, those experiments provided detailed information about HNDs, including their temperature [18], evidence for superfluidity [19], and the minimum number of helium atoms needed to establish superfluidity in droplets of ${ }^{4} \mathrm{He}$ [20].

Nowadays, a large number of research groups use nozzle beams to synthesize 
droplets made of ${ }^{4} \mathrm{He}$ (in this review we will not discuss ${ }^{3} \mathrm{He}$ droplets; these fermionic systems have properties very different from bosonic ${ }^{4} \mathrm{He}$ droplets). The droplets, typically containing $10^{3}$ to $10^{9}$ atoms, provide a unique environment to synthesize and interrogate atoms, molecules, and clusters. Bulk helium already offers some advantages to investigate dopants, including its optical transparency and its very weak interaction with the embedded species. HNDs add several additional advantages, including the absence of physical walls to which embedded species would want to migrate, and the absence of photodegradation because in a beam, doped droplets are rapidly replenished.

Several comprehensive reviews of research involving HNDs have been published [21-26]. Recent topical reviews in this journal and general articles elsewhere have focused on optical spectroscopy of doped HNDs [27-31], the dynamics upon excitation or ionization [32], and theoretical investigations of doped HNDs [33-35]. The ultrafast dynamics in photo-excited doped HNDs has been explored in [36-38]. Other opportunities in HND research include the synthesis of nanostructured objects [39-41] and metastable structures [42].

The present review focuses on the solvation of ions in HNDs, with an emphasis on topics pursued by its authors. After a brief summary of the properties of positive and negative charges in bulk helium (Section 2) we will discuss, in Section 3, methods to synthesize, dope and ionize HNDs. We will present evidence that small helium cluster ions containing tens to hundreds of helium atoms are the fission products from very large, highly charged HNDs. With few exceptions, most neutral dopants are known to reside in the interior of HNDs. Alkali metals, discussed in Section 4, are an exception: Atoms reside on the surface but helium wets the bulk surfaces of lithium through rubidium. Thus, depending on their size, alkali clusters will either reside on the surface or in the interior of HNDs. The size at which the transition occurs has been determined by measuring the ion yield of sodium and potassium clusters near the ionization threshold.

The concept of snowballs in bulk helium suggests that most ions solvated in the interior of HNDs will form a solid-like solvation shell. How many atoms do they contain? What is their structure? Their stability? Does the inclusion of quantum phenomena blur the distinction between solid-like and liquid-like? Do distinct second or even third solvation shells form? Tentative answers to these questions based on experimental data will be addressed in Section 5 which will also include a brief discussion of ion solvation in molecular hydrogen. Theory, to be discussed in Section 8, will provide more detailed insight.

Data compiled in Section 5 are mostly based on mass spectrometry. In Section 6 we review alternative methods used to grow $\mathrm{He}_{N} \mathrm{X}^{+}$cluster ions. Spectral shifts observed in the electronic absorption spectra of fullerene and corannulene cations complexed with up to 100 He atoms are discussed in Section 7; they provide detailed information about the location of adatoms in the adsorption layer. 


\section{Snowballs and bubbles}

\subsection{Early work on ions in liquid bulk helium}

The mobility $\mu$ of impurity ions in liquid ${ }^{4} \mathrm{He}$ is surprisingly low. If one uses the Nernst-Einstein relation to derive from $\mu$ the diffusion coefficient $D$ at 1.2 K, i.e. in superfluid helium, one finds values about two orders of magnitude smaller than the diffusion coefficient of ${ }^{3} \mathrm{He}$ in dilute ${ }^{3} \mathrm{He}-{ }^{4} \mathrm{He}$ mixtures [43]. In 1959 Atkins proposed that there is a large region surrounding the ion in which the density of the liquid is increased due to electrostriction. As a result, an ion exposed to an external electric field drags with it a large number of ${ }^{4} \mathrm{He}$ atoms and therefore has a very large effective mass [43]. Applying a continuum model he estimated that the pressure in the vicinity of the ion will greatly exceed the pressure at which liquid ${ }^{4} \mathrm{He}$ freezes and, consequently, the ion will be embedded in a large sphere of solid helium. Atkins conjectured that the sphere might contain roughly 40 to 50 helium atoms but could not provide a reliable estimate without knowing the surface tension between liquid and solid helium.

About a decade later Johnson and Glaberson developed a technique for introducing a variety of alkali and alkaline earth cations into liquid helium and measuring their mobility [44]. They coined the term "snowball" for the solid-like sphere of helium surrounding the ion, and proposed to think of the snowball as an ion surrounded by one or more discrete shells of helium atoms. They observed that the snowball radius depended not only on the nominal radius and charge of the impurity ion but also on its chemical nature. For alkali ions (and pure helium, for which the charge is localized on $\mathrm{He}_{2}^{+}$or $\mathrm{He}_{3}^{+}$[45]) the radius is about 7 to $8 \AA$ ¿ $[46,47]$.

Subsequent theoretical work, which will be discussed in detail in Section 8, has corroborated the basic ideas of the snowball model. However, whereas the long-range interaction between an ion and the polarized helium atoms is always attractive, the short-range interaction may be repulsive as a result of the Pauli exclusion principle. For example, while alkali cations produce a solid-like snowball structure, $\mathrm{Ca}^{+}$produces a liquid-like structure [48]. The effect of the exchange-repulsion is even stronger for some anions; their mobility in helium tends to be even lower than that of cations (see $[43,47,49]$ and references therein). The effect is most dramatic in the description of excess electrons in helium. In 1961 Kuper suggested that an electron in liquid ${ }^{4} \mathrm{He}$ resides in a cavity, or "bubble", of radius $12 \AA$ [50]. More recent work places the radius at about $17 \AA$ [51]. The radius of bubbles around anions with complete electronic shells, such as $\mathrm{Cl}^{-}$or $\mathrm{OH}^{-}(R=3$ to $4 \AA$ A $)$ is much smaller though $[49,52]$.

\subsection{Early beam studies of pure and doped helium droplets}

The first successful production of helium clusters in nozzle expansions of pre-cooled helium gas was reported by Becker and coworkers [1, 53]. The measurement of their size by mass spectrometry proved difficult because large neutral clusters, all traveling at the same speed of typically $200 \mathrm{~m} / \mathrm{s}$ (dependent on the nozzle temperature), carry a significant amount of kinetic energy. Furthermore, electron ionization may give rise to multiply charged ions and excessive fragmentation. A clever combination of various beam techniques eventually allowed Gspann and coworkers to measure the 
size distribution of neutral $\mathrm{He}_{N}$ clusters; average sizes were found to range from $\langle N\rangle=10^{3}$ to $10^{8}[3,5,54]$. Species in this size range are now commonly referred to as helium nanodroplets (HNDs). They will escape detection in conventional mass spectrometers because the kinetic energy of their neutral precursors will be roughly $N \times 10^{-3} \mathrm{eV}$; the applied electric or magnetic fields will be too weak to steer these ions towards the detector. However, the sudden introduction of a positive charge in helium (which will eventually terminate in the formation of a tightly bound $\mathrm{He}_{2}^{+}$, see Section 3) will be followed by electrostriction and possible ejection of a small cluster ion containing tens or hundreds helium atoms $[4,6]$. Following this pioneering work at the KFK Karlsruhe, the number of groups investigating the properties of HNDs, or using HNDs for matrix isolation spectroscopy or chemical reactions, grew rapidly. For comprehensive reviews of work done before $\sim 2001$ see refs. [13, 21, 55]; several more recent reviews have been published in the present journal [27-30, 32-35].

Although the concept of "snowballs" is frequently encountered in experimental reports on doped helium clusters, its meaning is ill-defined. Toennies and coworkers have used the term to refer to the dense, incompressible region of solvent atoms around an embedded neutral $\mathrm{SF}_{6}$ molecule, as deduced from rotationally resolved IR spectroscopy [56]. Ellis and coworkers have invoked the term to describe the tightly bound solvation shell (or cage) that reduces the ionization-induced escape of charged molecular fragments to a much greater extent than the escape of neutral hydrogen atoms [57]. In mass spectrometric work, the term snowball often refers to any ions of the form $\mathrm{He}_{N} \mathrm{X}^{+}$where $\mathrm{X}$ is the original dopant or a fragment thereof [58-61]. In our present discussion of mass spectra (Section 5) we will avoid the term, although we will argue that local anomalies in the abundance distribution of $\mathrm{He}_{N} \mathrm{X}^{+}$indicate structural order.

In cluster research described in the present review, the exchange-repulsion mentioned above has two notable effects: First, it affects the location of neutral dopants in HNDs. Whereas most neutral dopants will be fully solvated within the HND, alkali atoms and their clusters, if sufficiently small, will reside on the droplet surface. In Section 4 we will discuss attempts to determine the size of the dopant cluster at which the interior site will be preferred versus a surface site. Second, the large bubble formed around excess electrons in helium implies that small negatively charged $\mathrm{He}_{N}^{-}$ do not exist (unless the electron resides on a metastable, electronically excited helium atom, see Section 3). Electron bubbles are, in general, not thermodynamically stable; their energy lies above the energy of a HND (even if their size $N$ approaches infinity) plus a electron in vacuum. A bubble is metastable, though, if its energy lies below the bottom of the electron conduction band. For this stability criterion Rosenblit and Jortner have estimated a minimum cluster size of $N=5200$ [51]. The smallest $\mathrm{He}_{N}^{-}$ observed by Henne and Toennies upon attachment of low-energy electrons to HNDs contained $7.5 \times 10^{4}$ helium atoms, equivalent to a droplet radius of about $93 \AA$ [62]. Conceivably, an excess electron may also bind to a HND in a surface bound state for which Rama Krishna and Whaley have estimated a minimum cluster size of $5 \times 10^{5}$ atoms [63]. However, the calculated binding energy of these states is very weak; the fact that the measured lifetime of $\mathrm{He}_{N}^{-}$exposed to strong electric fields exceeds the experimental time scale seems to rule out the importance of surface bound states to 
experiments reported so far [64]. Further details about undoped negatively charged helium clusters may be found in [26].

Any discussion of the observability of weakly bound anions and of structural order (solid versus liquid) raises, of course, the question of temperature. Brink and Stringari have estimated that HNDs (which are "boiling hot" when formed in the nozzle expansion) will rapidly cool to about $0.4 \mathrm{~K}$ on the time scale of typical beam experiments (micro- to milliseconds) [10]. The value is in excellent agreement with the temperature of $0.37 \mathrm{~K}$ determined by rotationally resolved IR spectroscopy of $\mathrm{SF}_{6}$ embedded in HNDs [56, 65]. Furthermore, at this temperature the droplet is superfluid; a cluster containing roughly $60{ }^{4} \mathrm{He}$ atoms (embedded in a HND composed of ${ }^{3} \mathrm{He}$ ) is sufficient for the manifestation of superfluidity [65].

In this review we are concerned with charged clusters. Their temperature is expected to be similar to that of neutral HNDs if they are sufficiently large, i.e. if their evaporation energy approaches the bulk value, $D_{\infty}=0.616 \mathrm{meV}$ (see [51] and references therein). For small $\mathrm{He}_{N} \mathrm{X}^{+}$the evaporation energy $D_{N}$ will be significantly larger than the bulk value, and their temperature will be correspondingly higher. Note, however, that the concept of temperature for small, isolated clusters in a beam is subtle because they do not form a canonical ensemble, nor do they constitute their own heat bath $[66,67]$. This topic will be explored further in Section 5 .

\section{Experimental conditions for the efficient synthesis of snowballs and ionization of helium nanodroplets}

\subsection{Formation of HNDs}

The most common method to form HNDs is free-jet expansion of pressurized and precooled super-clean (99.9999\% purity) helium [13, 21, 26]. In order to prevent shock waves that destroy the weakly-bound droplets, skimmers and differential pumping are required. Pulsed [68-75] as well as continuous [76-80] nozzles are utilized for helium droplet production. The benefit of pulsed nozzles is a strongly reduced gas load and therefore, smaller and cheaper vacuum pumps can be used. Furthermore, the consumption of helium is reduced (a typical continuous nozzle with a diameter of the pinhole of $5 \mu \mathrm{m}$ consumes about $10 \mathrm{~m}^{3}$ He per month) and the peak flux of droplets can be up to a factor 1000 higher than for continuous sources [70]. Lower stability [68] and less homogeneous velocity distributions [70] are downsides of pulsed nozzles. In addition, the average droplet size changes between the pulses and even within each pulse [81]. Better size definition, stability, and coupling to subsequent experiments (either continuous or pulsed with up to $100 \mathrm{kHz}$ ), makes the continuous nozzle type our preferred helium droplet source.

The size distribution of the neutral helium droplets depends strongly on the temperature of the droplet source $[21,78,82,83]$. The desired temperature is reached by permanent cooling (closed cycle [14, 84-86], continuous flow [21, 76, 87] or liquid helium bath [88] cryostats) and counter heating with a (PID) temperature control loop. When mounting a helium droplet source directly to the $4 \mathrm{~K}$ cooling stage of a pulse tube cryocooler (Sumitomo Heavy Industries, model RP-182B2S with a cooling power of $1.5 \mathrm{~W}$ at $4.2 \mathrm{~K}$ ) with an indium sheet between the two parts, the low-specific 
heat and high thermal conductivity of the oxygen free copper (for the sources in Innsbruck copper from Montanwerke Brixlegg MB-OF101 with a residual-resistance ratio RRR > 200) used for the HND source in combination with the periodic cooling cycles of the closed cycle cryo-cooler lead to temperature oscillations up to $0.5 \mathrm{~K}$, resulting in unacceptably large oscillations of the droplet size and ion yield. From the thermodynamic consideration, both heat capacity and thermal conductance should be large in order to reduce the temperature oscillations without compromising cooling power $[89,90]$. By placing a stainless-steel sheet between the cold head and the HND source, we were able to reduce the oscillations below $\pm 0.04 \mathrm{~K}$. At this low temperature, even such small temperature variations have a noticeable effect on several quantities to be measured. For closed cycle cryocoolers of the Gifford-McMahoon-type, also mechanical oscillations with the frequency of the cooling cycle additionally enhance fluctuations of the resulting droplet jet, but they are strongly reduced for pulse tube coolers. Figure 1 shows the temporal dependence of the ion yield of multiply-charged HNDs as well as the pressure in the droplet source (before the skimmer) and the ion source (after the skimmer) and the temperature close to the nozzle as a function of the time. Gómez et al. [78], the average neutral droplet size for the present conditions (4 K source temperature, $2 \mathrm{MPa}$ He pressure, $5 \mu \mathrm{m}$ nozzle diameter) is expected to contain more than $10^{10}$ He atoms. After passing a skimmer with an orifice of $0.5 \mathrm{~mm}$ the neutral droplets are crossed with an electron beam $(94 \mu \mathrm{A}$ electron current and $160 \mathrm{eV}$ electron energy). With a spherical electrostatic sector field analyzer charged HNDs containing 2.3 million He atoms per charge are selected and detected with a single particle detector. This indicates charge states in the order of several 1000 . The periodic oscillations on the heavy charged HND signal is about $3 \%$ of the average value. However, for more gentle ionization conditions (lower electron current and electron energy) and higher source temperatures the oscillations can reach up to $50 \%$ of the average signal. Optimization of the PID control loop can reduce the oscillations at these elevated temperatures to about $10 \%$.

\subsection{Ionization of HNDs}

Photoionization of HNDs requires special light sources due to the high ionization energy of helium and is studied by several groups at free electron lasers [38, 91], synchrotrons [92-94] and laser-based extreme ultraviolet sources [32]. Pickup of ions into neutral HNDs has been achieved in linear ions traps [75, 95] merged [96] or crossed beams [97], and by passing the neutral HND beam near the surface of a metal exposed to a pulsed laser beam [98]. Electron ionization is by far the most common ionization method [26], which also enables the formation of negatively charged ions $[99,100]$. For HNDs containing fewer than $10^{5}$ helium atoms (which corresponds to a radius of $10.6 \mathrm{~nm}$ ), a uniform probability for the ionization of a random He atom can be expected and for larger droplets the electrons will preferentially ionize He atoms in the surface region of the HNDs. $\mathrm{He}_{2}^{+}$is a covalently bound molecule with a binding energy of $2.35 \mathrm{eV}$ [101]. However, the formation of this ground state dimer cation from an initially formed $\mathrm{He}^{+}$and a neighboring $\mathrm{He}$ atom is much slower than resonant electron transfer to neighboring atoms. Such a resonant charge transfer mechanism 


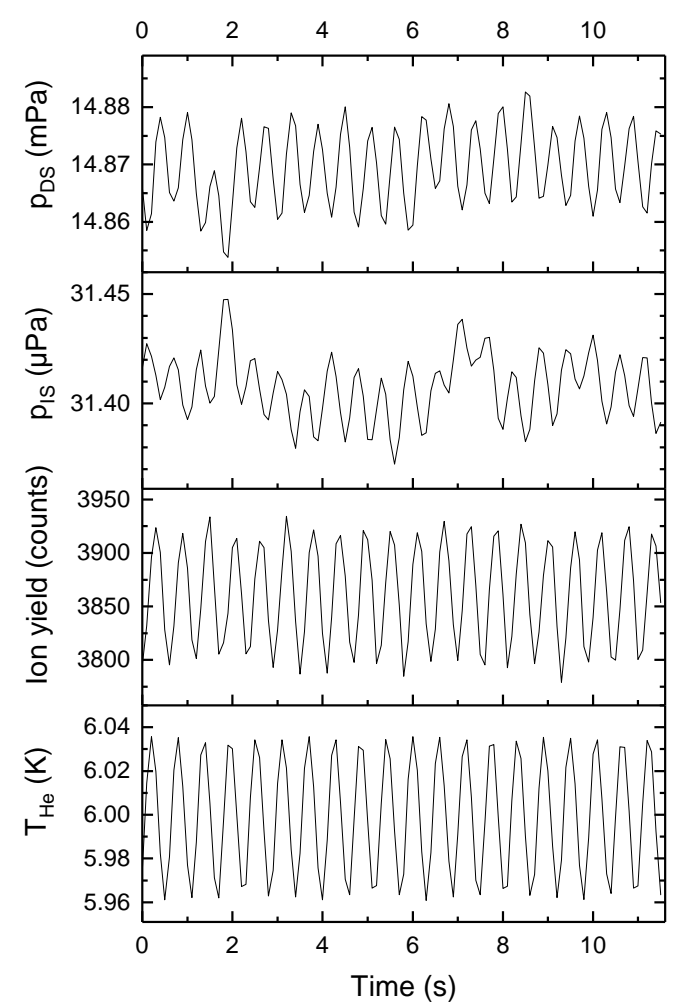

Figure 1. Effect of periodic temperature oscillations on the pressure before and after the skimmer and the ion signal of multiply-charged helium droplets containing 2.3 million He atoms per charge.

was introduced by Atkins [102] and further developed by Buchenau et al. [103] in the case of helium droplets. A calculation of the mean time between subsequent electron transfers resulted in a value of the order 20 fs [104]. On this time scale the motion of the atomic nuclei is small and the charge may be assumed to hop several times before dimerization takes place. Based on measurements with doped HNDs a more elaborate model of the hole-hopping mechanism has been developed [105]. Dopants are more polarizable than He and therefore, the migration of the charge is directed towards the dopant [106]. From a classical model a mean free path of $34 \mathrm{~A}$ for the charge hopping in helium was calculated, which corresponds to approximately 11 hops before $\mathrm{He}_{2}^{+}$ is formed. This is similar to the hopping numbers deduced by other research groups using different models [106]. Charge transfer to a dopant also terminates the hole hopping mechanism.

\subsection{Mass spectra upon ionization of pure and doped HNDs}

Mass spectra of positively charged ions formed upon electron ionization of HNDs always show a series of $\mathrm{He}_{N}^{+}$ions with some intensity anomalies below $N=30$ and an almost exponential decrease for larger $N$ (see Figure 2). In the case of doped HNDs, 

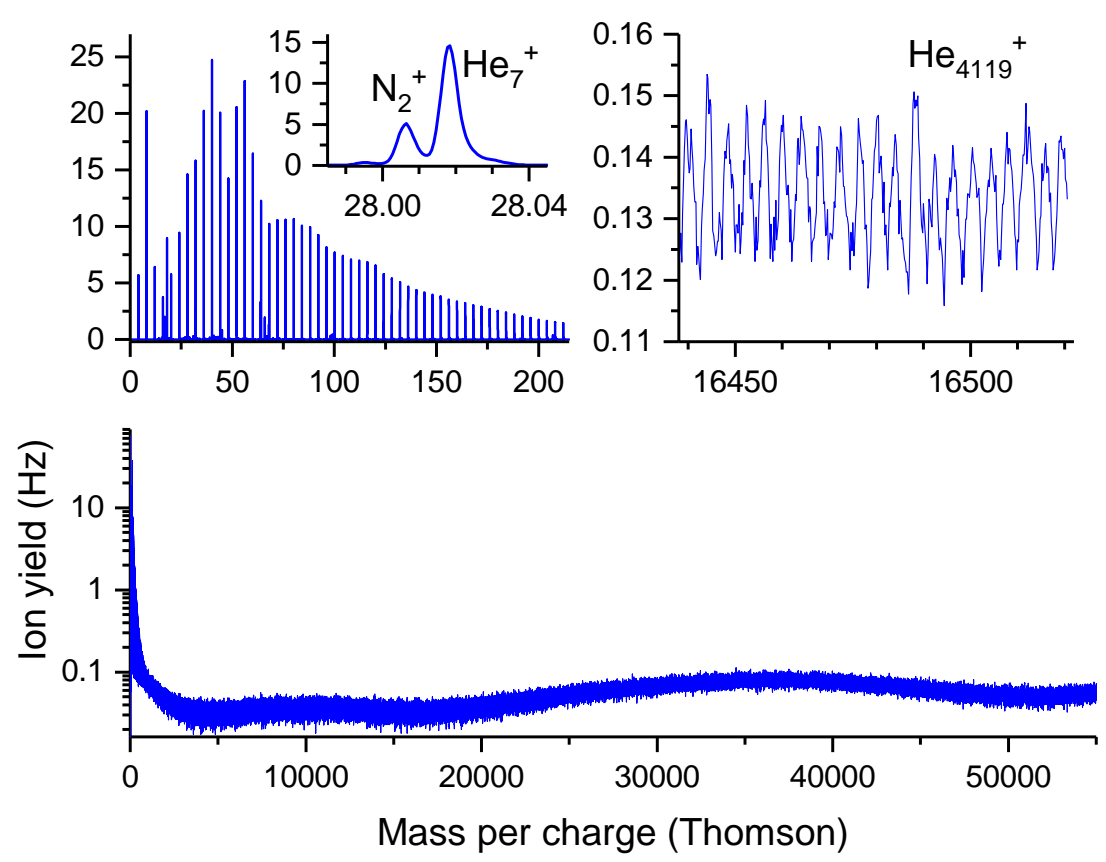

Figure 2. Sections of mass spectra of cations formed upon electron ionization of undoped HNDs. Parameters: He temperature $8.5 \mathrm{~K}$, He pressure 20 bar, average neutral droplet size $\langle N\rangle=3 \times 10^{6}$ [78]; electron energy $100 \mathrm{eV}$, electron current $0.4 \mu \mathrm{A}$.

additional ion series of pure dopant clusters and with typically much lower intensity also mixed dopant-helium clusters are observed. Mass spectra of cluster ions often exhibit intensity anomalies or pronounced signal drops at particular cluster sizes which are often referred to as magic numbers or shell closures, respectively. In the present review, the completion of the first solvation layer of helium around a dopant ion $\mathrm{X}^{ \pm}$ is experimentally determined from magic numbers or shell closures in the ion series $\mathrm{He}_{N} \mathrm{X}^{ \pm}$. Only mass spectrometer systems that measure simultaneously the whole mass range are not affected by instabilities of the cluster formation processes, such as temperature oscillations (see Figure 1), which makes time of flight mass spectrometer systems (TOF-MS) the preferred instruments. Furthermore, low-mass dopant ions and their corresponding He solvated ions are often isobaric with other ions, including residual gas ions. One way to separate most of these ions is a sufficiently high resolving power of the mass spectrometer system. Three reflectron TOF instruments utilized in Innsbruck achieve mass resolution values $m / \Delta m$ in the $\mathrm{V}$-mode between 6000 [80] and 17000 [107].

Figure 2 shows a typical mass spectrum of $\mathrm{He}_{N}^{+}$ions formed upon electron ionization of neutral HNDs with an average size of about three million He atoms. Small cluster ions exhibit a few magic numbers at $N=7,10$, and 14, and very weakly for $N=$ 23, 30 that agree well with intensity anomalies reported in the literature [26, 108]. An enlarged section of the mass range around $m / z=28$ demonstrates that the ion signal consists of several contributions, including $\mathrm{He}_{7}^{+}, \mathrm{N}_{2}^{+}, \mathrm{CO}^{+}$and $\mathrm{C}_{2} \mathrm{H}_{4}^{+}$, with 
the $\mathrm{He}_{7}^{+}$being the dominant ion. However, even a tiny leak (in the present case the pressure in the ion source region is below $10^{-6} \mathrm{~Pa}$ with the He beam on) makes $\mathrm{N}_{2}^{+}$ the most abundant species. The ion yield of the $\mathrm{He}_{N}^{+}$series exponentially decreases up to about $N=150$ and with a smaller exponential decay rate up to $N=1000$. For larger ions, the signal exhibits two wide peaks around $N=2500$ and $N=9000$ and it increases again after $N=13000$ up to the end of the mass range of this spectrum. The enlarged section at around $N=4120$ clearly shows that the signal results from $\mathrm{He}_{N}^{+}$ions that are separated by about four mass units. Please note, that even the heaviest ions in this measurement contain less than $1 \%$ of the average neutral droplet size.

\subsection{Large charged HNDs}

Only very few experiments have been performed that analyzed charged helium droplets containing up to several million He atoms. Single particle detectors, such as secondary electron multipliers or multi-channel plates, require typically fast ions that upon collision with the detector surface emit a first electron (kinetic emission [109]). Ions with a potential energy that exceeds twice the work function of the detector material are able to emit electrons even at very low velocities (potential emission [110]). The high ionization energy of helium enables potential emission, down to the low velocities of helium droplet jets formed at the lowest nozzle temperatures. Normal mass spectrometers are not capable to handle such massive ions and therefore different techniques, such as retarding field analysis or deflection methods were utilized. Both methods take advantage of the narrow velocity distribution of free jet expansions and therefore the kinetic energy is proportional to the mass of a droplet. The velocity of a free jet depends on the temperature of the stagnant gas [76, 78, 83]. HNDs that are formed upon expansion of He at $6 \mathrm{~K}$ have a velocity of $176 \mathrm{~m} / \mathrm{s}$ and an average size of $3.08 \times 10^{8} \mathrm{He}$ atoms [78]. The kinetic energy of such droplets is about $200 \mathrm{keV}$, which requires enormous electrical fields and/or large deflectors (sector fields with large radii or long parallel plates) to deflect these ions. With a cylindrical sector field analyzer having a radius of $5 \mathrm{~m}$ and a distance between the deflector plates of $1 \mathrm{~cm}$, a moderate voltage of about $\pm 400 \mathrm{~V}$ has to be applied to pass singly charged droplets containing $3 \times 10^{8} \mathrm{He}$ atoms at a velocity of $176 \mathrm{~m} / \mathrm{s}$ through this energy analyzer. Another issue in mass spectrometry of massive ions is the fact that the yield of secondary electrons generated upon ion impact on the conversion surface of the detector usually drops to zero below a threshold velocity of about $10^{4}$ $\mathrm{m} / \mathrm{s}$ [111]. However, the neutralization energy of a charged HND greatly exceeds the work function of typical surfaces, resulting in non-zero conversion efficiencies well below the threshold velocity.

\subsection{Multiply-charged HNDs}

Laimer et al. recently discovered that pristine HNDs when multiply ionized can accommodate a surprisingly large density of charges close to their surface and eject only small ions if charged beyond a critical value [112]. There is good evidence that all low-mass ions measured by mass spectrometry upon electron ionization of large 
(doped and undoped) HNDs are the result of such asymmetric Coulomb explosions of multiply-charged HNDs. Furthermore, the majority of He and dopants are most likely remaining in a large multiply-charged droplet that cannot be detected by conventional mass spectrometry. Tiefenthaler et al. managed to extract ionic cores from multiply-charged droplets via evaporation of the helium matrix in a radiofrequency ion guide upon multiple collisions with room temperature helium gas [107]. Depending on the pressure of the helium gas, it is possible to obtain bare dopant cluster ions or ions that still are solvated with some He atoms and the number of He small enough to detect these ions with a TOF-MS. As long as cooling of ions via evaporation of He is active, the temperature of the dopant cluster ions remains at the temperature of the He matrix but will increase with increasing binding energy of the least tightly bound He atoms (See Section 5.1).

\subsection{Efficiency of snowball formation}

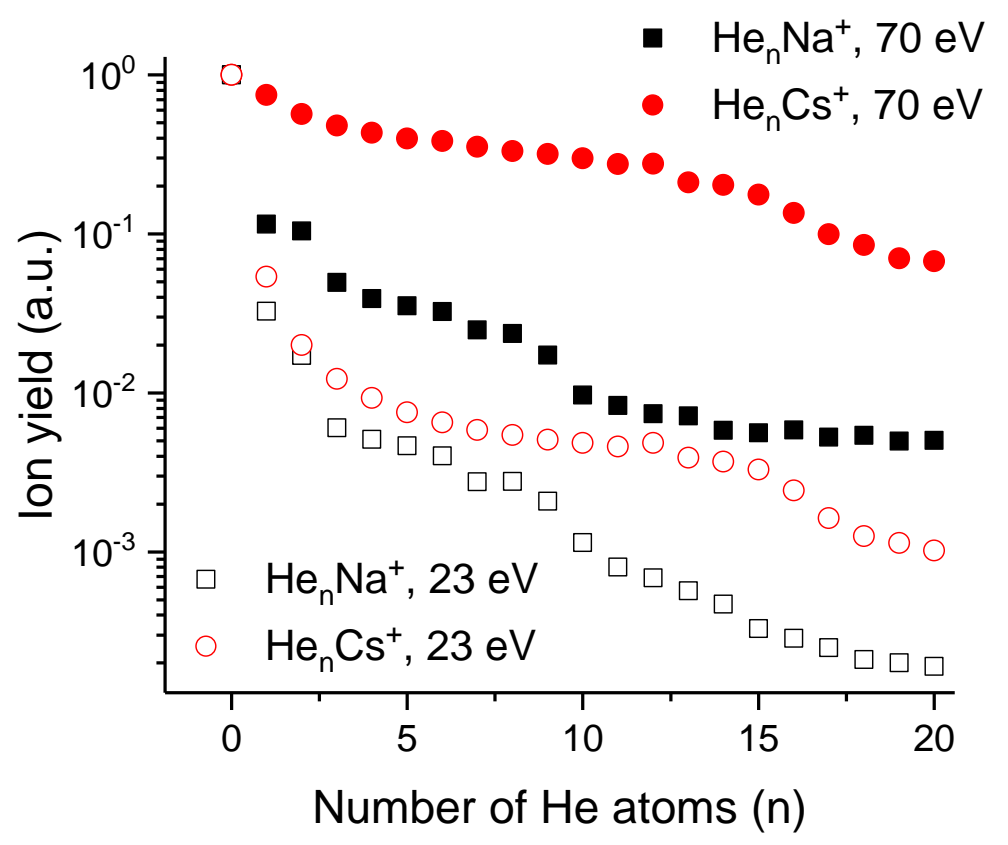

Figure 3. Cluster size distribution of helium solvated $\mathrm{Na}^{+}$(black squares) and $\mathrm{Cs}^{+}$(red circles) at two different electron energies. At $70 \mathrm{eV}$ (solid symbols) the preferential ionization mechanism is charge transfer from $\mathrm{He}^{+}$or $\mathrm{He}_{2}^{+}$and at $23 \mathrm{eV}$ (open symbols), Penning ionization via $\mathrm{He}^{*}$ is the only possible mechanism.

In contrast to cryogenic ion traps [113-116] and drift tubes [117-120], where the number of He atoms attached to the ions is rather limited, ions from doped HNDs can be solvated by many He atoms [108, 121-123]. For more than 20 years He solvated ions have been formed upon electron ionization of doped HNDs [108]. Depending on the dopant under investigation, the amount of dopant ions complexed with He 
can differ dramatically. For large molecular ions, such as fullerenes, the bare ions are orders of magnitude more intense than the integrated ion yield of all He complexed ions $[124,125]$. In contrast, for heavy atomic ions, such as $\mathrm{Au}^{+}[126], \mathrm{I}^{+}[127] \mathrm{or} \mathrm{Cs}^{+}$, the situation can be the opposite, when tuning the instrument for He tagged ions. In Figure 3 the ion yield of helium solvated sodium and cesium cations is shown for high $(70 \mathrm{eV})$ and low $(23 \mathrm{eV})$ electron energies. Please note that the mass of the dopant as well as the ionization process have a strong influence on the relative abundance of helium solvated ions. Another important parameter for efficient formation of $\mathrm{He}$ solvated ions turns out to be the settings of the ion source. The upper two diagrams in Figure 4 show two mass spectra obtained upon electron ionization of the same neutral distribution of $\mathrm{C}_{60}$ doped HNDs for different ion source settings. However, even under perfect conditions, the amount of the bare $\mathrm{C}_{60}^{+}$ion is orders of magnitude more abundant than the most intense $\mathrm{He}_{N} \mathrm{C}_{60}^{+}$complex.

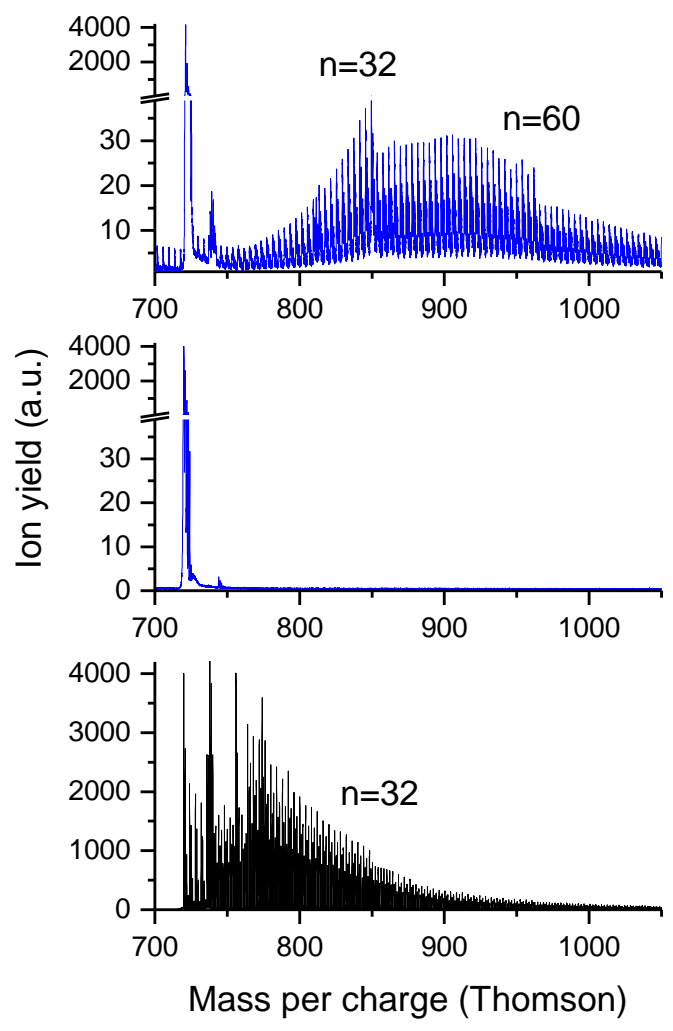

Figure 4. Sections of mass spectra showing $\mathrm{He}_{N} \mathrm{C}_{60}^{+}$formed upon electron ionization of neutral HNDs doped with $\mathrm{C}_{60}$ (upper two diagrams) and pickup of $\mathrm{C}_{60}$ into multiply-charged HNDs (lower diagram). The upper diagram was taken under perfect conditions for the formation of He tagged ions $\mathrm{He}_{N} \mathrm{C}_{60}^{+}$whereas the middle diagram is a typical mass spectrum, optimized for fullerene cluster ions $\left(\mathrm{C}_{60}\right)_{N}^{+}$. Intense peaks in the lower diagram can be assigned to small water impurities (about $1 \mathrm{ppm})$ in the He gas used to evaporate excess He. These water molecules attach efficiently to the fullerene ions. Nevertheless, a clear intensity drop can be observed after $\mathrm{He}_{32} \mathrm{C}_{60}^{+}$. 
Tiefenthaler et al. [107] recently pioneered an alternative method where multiplycharged pristine HNDs are doped with atomic or molecular vapors. The dopants are polarized and attracted by the ionic centers that are located uniformly distributed close to the surface of the HNDs. Evaporation of the helium matrix by multiple collisions with room temperature helium in a radio frequency ion guide extracts the ions from the droplet. Depending on the He gas pressure in this collision cell, the ions are still solvated with a preferred number of He atoms. The lowest diagram in Figure 4 shows a mass spectrum obtained by this novel technique. The amount of He tagged ions is about three orders of magnitude higher than before.

\section{Impurity location}

One of the main concerns in the study of doped helium clusters regards the specific position of the impurity with respect to the solvating atoms. For the case of neutral species, usually, this information may be inferred via the comparison between the electronic absorption spectrum and measurements made in either bulk or gas phase. The shift and width of the corresponding electronic transitions are then good indicators of the structure and the precise location of the dopant atom. There is a extense variety of cases and thus, one can find the example of $\mathrm{Ag}$ [128], which gets trapped inside the helium droplet, whereas alkaline atoms ( $\mathrm{Li}, \mathrm{Na}$ or $\mathrm{K}$ ) seem to prefer an outside position occupying a dimple at the surface of the cluster [129]. A similar behaviour is observed for alkaline earth metals such as $\mathrm{Ca}, \mathrm{Sr}$ or $\mathrm{Ba}[130,131]$. Nanodroplets in the presence of $\mathrm{Mg}$, on the other hand, have been the subject of certain controversy regarding the position of the dopant atom [132, 133]. The actual composition of the helium nanodroplet can be a determinant factor, since impurities can behave differently inmersed in ${ }^{3} \mathrm{He}$ or ${ }^{4} \mathrm{He}$ medium. Ca atoms, for instance, are completely solvated in pure ${ }^{3} \mathrm{He}$ clusters but, in the case of mixed ${ }^{3} \mathrm{He} /{ }^{4} \mathrm{He}$ samples, they can only penetrate the fermionic shell and get stopped once they find the bosonic core [134-136]. Features of this distinct behaviour depending on the isotopic variant of He can be also observed for the smallest clusters [137]. The importance of the description of the interaction between He and the corresponding neutral dopant in theoretical investigations of this kind of clusters has been examined, for the case of Ca, in previous studies [138].

Spectroscopic methods have provided information about the location of alkali atoms and diatomics on the surface of a HND (supposed to be composed of ${ }^{4} \mathrm{He}$ from here on) [30]. Furthermore, techniques such as time-resolved multiphoton excitation and ion imaging have been applied to elucidate the dynamics of desorption upon excitation or immersion upon ionization [30, 61, 139-141]. But what about clusters of alkali atoms? Probing the properties of clusters as a function of their size is particularly intriguing if a large, perhaps even qualitative change is to be expected between the molecular regime and the bulk [142]. Such a profound change is anticipated for the alkali-helium system because, while HNDs and bulk helium do not solvate alkali atoms, liquid helium is known to wet the surfaces of Li, Na, K and Rb metals. (For Cs the exchange-repulsion is particularly strong because its very small work function implies that the wavefunctions of its conduction electrons extend far out into the 
vacuum region; wetting does not occur at very low temperatures $[143,144])$. Thus, for sufficiently large clusters of alkalis (possibly barring Cs) in sufficiently large HNDs the solvated (interior) position should become energetically lower than the surface state.

How does one probe the expected transition? Spectroscopic techniques will be blunted because the absorption spectrum of large metal clusters tends to become broad and featureless [145-147]. The very weak perturbation by the helium matrix, usually viewed as an advantage, will make it near-impossible to distinguish between surface and interior states. In our lab at Innsbruck we have pursued another approach, by analyzing the ion yield in the vicinity of the ionization threshold of HNDs doped with alkalis. As discussed in Section 3, there are two main channels for the production of positively charged helium clusters by energetic electrons [26]. Process (1) starts with the formation of $\mathrm{He}^{+}$which will hop to other He atoms by resonant charge transfer. Charge hopping terminates upon the irreversible formation of a tightly bound $\mathrm{He}_{2}^{+}$(binding energy $2.35 \mathrm{eV}$ ). In pure HNDs this heliophilic species will be solvated near the cluster core. In doped HNDs, $\mathrm{He}_{2}^{+}$will efficiently ionize a dopant that resides in the interior of the droplet via charge transfer.

Process (2) starts with the formation $\mathrm{He}^{*}$, an electronically excited helium atom. For free atomic He the process has a threshold of $19.8 \mathrm{eV}$ (He* excited to the 2 ${ }^{3} S$ state). In a droplet the threshold is raised by about $1 \mathrm{eV}$, the energy difference between the bottom of the conduction band and the vacuum level [51]. He* is heliophobic and will reside near the surface of the droplet. In doped clusters $\mathrm{He}^{*}$ may form a positive ion via Penning ionization if the dopant is heliophobic as well.

Thus, the telltale of a dopant residing in the interior of a large HND is an ionization threshold not much less than the threshold for that of free helium atoms $(24.59 \mathrm{eV})$. In contrast, the telltale of a dopant residing on the surface would be a reduced ionization threshold consistent with the formation of He*. Penning ionization had already been utilized by Kresin and coworkers to demonstrate that Li, Mg, Ca, Sr, and $\mathrm{Ba}$ atoms and $\mathrm{Na}$ clusters containing up to 13 atoms all reside at or near the droplet surface. However, they did not explore the transition from surface to interior location [148-150]. Note that a third conceivable process, direct ionization of the dopant by an incident electron, would feature a much lower threshold, especially for alkalis. The efficiency of this process, though, is statistically insignificant for large HNDs; its contribution to the measured ion yield is negligible.

Fig. 5 displays the relative yield of $\mathrm{K}_{N}^{+}$cluster ions, formed by electron ionization of doped HNDs, as a function of the incident electron energy [151]. The estimated size of the HNDs was $10^{5}$; the energy resolution of the electron beam was about 1 $\mathrm{eV}$. The yield curves are normalized at $27.5 \mathrm{eV}$ in order to enhance the visibility of features in the threshold region, the smooth curves are drawn to guide the eye.

The ion yield curves for the smaller clusters rise relatively sharply at about $20 \mathrm{eV}$, well below the threshold for $\mathrm{He}^{+}$formation $(24.59 \mathrm{eV})$. This is consistent with the onset of Penning ionization. Above $\sim 21 \mathrm{eV}$, the signal seems to plateau to some extent and then begins to rise again, with a clear increase in slope above $\sim 24 \mathrm{eV}$. This second rise is consistent with the onset of $\mathrm{He}^{+}$formation. However, for larger clusters $(N=80,90)$, the first (low-energy) component is missing. Also note that 
the shape of the first component, with a local maximum around $21.5 \mathrm{eV}$, signals a resonance (formation of $\mathrm{He}^{*}$ ) while the gradual increase of the second component is more typical of a vertical ionization process, with a poor Franck-Condon overlap of the neutral and the charged system.

Thus, the data in Fig. 5 suggest that small $\mathrm{K}_{N}^{+}$, but not large ones, can be ionized via formation of the heliophobic He*. In other words, the neutral precursors of the observed small $\mathrm{K}_{N}^{+}$reside on the surface of the HNDs whereas the neutral precursors of large $\mathrm{K}_{N}^{+}$reside in the interior. In order to determine the size of this structural transition more accurately we have parametrized the ion yield curves of the two components. The transition is then found to occur between $\mathrm{K}_{65}^{+}$and $\mathrm{K}_{80}^{+}$[151]. Similar experiments with sodium-doped HNDs place the transition size at $N=21$ [152]. The values are in excellent agreement with a model by Stark and Kresin who have analyzed the competition between the metal-helium van der Waals attraction on one hand, and the costs of the short-range repulsion and the surface tension of the surrounding bubble on the other hand [153]. They estimate that full submersion becomes energetically favorable beyond a critical size $N=21$ and 78 for $\mathrm{Na}$ and $\mathrm{K}$, respectively.

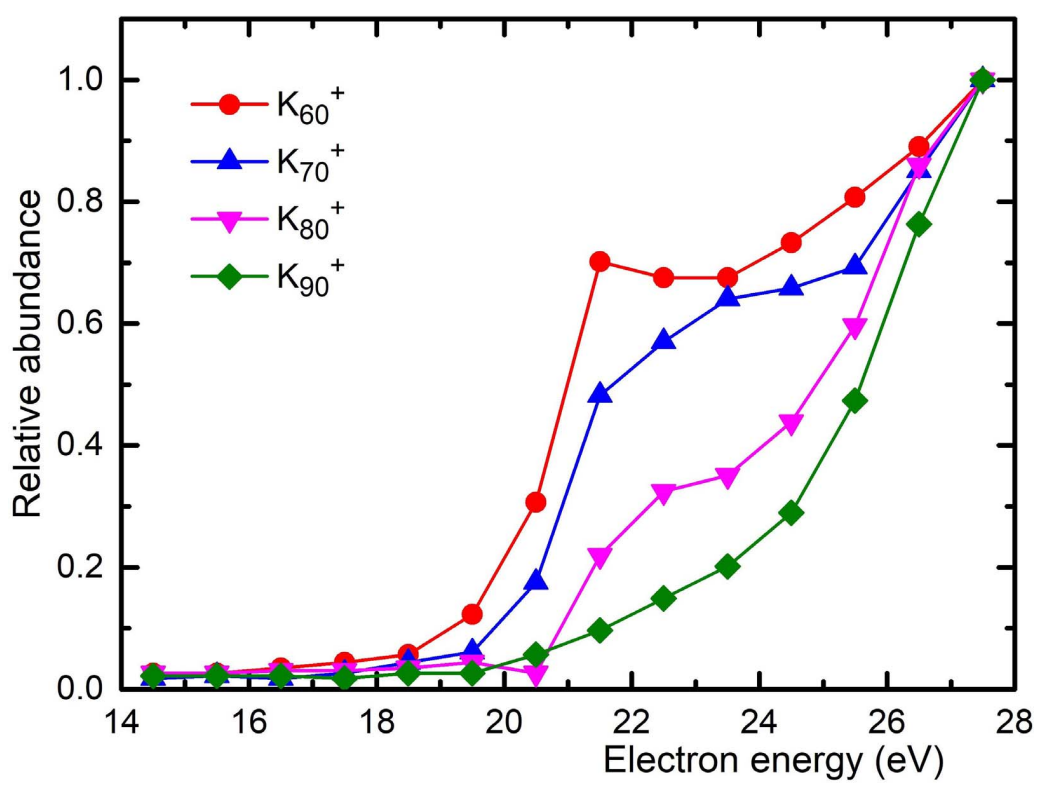

Figure 5. Ion yield curves as a function of electron energy for selected $\mathrm{K}_{N}^{+}$cluster ions $(N=60$, 70,80 , and 90). The curves were normalized such that the ion yields are the same at the maximum incident electron energy used $(27.5 \mathrm{eV})$.

A few comments are in order. First, the continuum model proposed by Stark and Kresin assumes a plane surface of helium, i.e. an infinite droplet size. A path-integral molecular dynamics (PIMD) study of $\mathrm{Na}_{N}$ in or on HNDs by Calvo shows that small droplets containing fewer than about $10^{5}$ particles tend to disfavor submersion [154]. Zillich and coworkers, in their path-integral Monte Carlo (PIMC) calculation 
of (rather small) HNDs, also find a strong effect of their size on their ability to wet a sodium cluster [155].

Second, the metal cluster is represented by a sphere. In the experiment, neutral metal clusters are grown by successive capture of alkali atoms by doped HNDs in the pickup cell. The heat of condensation (approximately $0.9 \mathrm{eV}$, the cohesive energy of the alkali metals in question) released upon addition of an atom to a cluster containing more than, say, a dozen atoms will not be sufficient to anneal the cluster into its new global minimum. As a result, the ultimate ensemble of clusters of size $N$ that are exposed to the incident electron beam will have a variety of structures and shapes. Consequently, the critical size at which a fully immersed cluster is energetically below a cluster on the surface will be distributed over a range of sizes.

Third, in the experiment one determines the size of the product metal cluster ions rather than that of the neutral precursors which will be larger if ionization induces fragmentation of the metal cluster. Fragmentation undoubtedly occurs in the experiments discussed here $[151,152]$, as evidenced by the absence of any helium atoms attached to the observed ions, and the appearance of local anomalies in the abundance distributions of $\mathrm{Na}_{N}^{+}$and $\mathrm{K}_{N}^{+}$that mirror the predicted high stability of cluster ions with closed electronic shells (e.g. at $N=9,21,41,59,93)$ and even numbers of electrons $[156,157]$. However, fragmentation is not likely to significantly underestimate the critical size determined in these experiments. While charge transfer from $\mathrm{He}_{2}^{+}$to an embedded metal cluster will release a large amount of excess energy and cause significant fragmentation, Penning ionization should be relatively soft since the departing electron can remove most of the excess energy delivered by He* . Because the presence of Penning ionization is the dominant criterion that is used for determining the critical size, the value deduced for the size of charged clusters should closely reflect the size of their neutral precursors.

Fourth, our assumption that an alkali cluster immersed in the interior of a HND cannot be Penning ionized is at odds with recent photoionization studies that suggest that dopants such as xenon, organic molecules, and their clusters can be efficiently Penning ionized even if they are immersed in the interior of the HND [158, 159]. Even so, the corresponding ion signal is suppressed because the nascent ions remain solvated; they are not detected as bare ions. Thus our conclusion will not change: The ion yield measured below the ionization energy of atomic He drops once the alkali cluster is immersed in the droplet.

As happens often in science: In answering one question we have raised a new one: If an alkali cluster has grown on the surface of a HND beyond its critical size and dived into the interior, what happens when another alkali atom is captured by the HND? The droplets spend about $0.3 \mathrm{~ms}$ in the pickup cell, hence the average time between capture of individual alkali atoms is $\gg 1 \mu \mathrm{s}$, plenty of time for the doped droplet to equilibrate after dopant pick-up before capture of another atom (or of ionization). Will the van der Waals interaction of the heliophobic atom with the cluster in the interior be strong enough to suck it into the HND? Will the metal cluster first have to move close to the atom on the surface? Or will the atom act as a nucleus for growth of a second cluster which will eventually merge with the first one?

These questions have not yet been addressed, neither by experimentalists nor by 
theorists. However, at Innsbruck we have addressed a related question: What happens if the HND is first doped with a highly polarizable entity (which will move into the interior) and, subsequently, with alkali atoms [160]? In the experiment the HNDs (estimated average size $5 \times 10^{5}$ atoms) are first doped with one or more $\mathrm{C}_{60}$ and then, in another pickup cell, with either sodium or cesium atoms. The yield of various ions is then recorded as a function of electron energy. The yield of $\left(\mathrm{C}_{60}\right)_{2} \mathrm{Na}_{N}^{+}$was consistent with ionization via $\mathrm{He}^{+}$formation for all values of $N \geq 0$, i.e. the neutral precursors reside in the interior. For Cs doped droplets the result was similar except for $\left(\mathrm{C}_{60}\right)_{2} \mathrm{Cs}^{+}$, these ions were predominantly formed via Penning ionization with a steep onset at $22 \mathrm{eV}$. Thus, the attraction between a $\mathrm{C}_{60}$ complex in the interior of the droplet and a single Cs atom was not strong enough to pull the metal atom into the droplet. Interestingly, the experimental findings did not change if the yield of ions containing a single, or multiple $(N>2)$ fullerenes. The authors used density functional theory to predict the potential energy curves between the dopants. They conclude that the $\mathrm{C}_{60}$ complex will be coated by a firm layer of helium atoms which will impede direct contact with the alkali atom, but that long range electron transfer will occur once the species are sufficiently close. The resulting strong attraction between the ion pair then leads to displacement of the helium that surrounds the reactants.

\section{Cluster size distributions}

\subsection{The relation between abundance and stability of solvated ions}

Mass spectra of atomic or molecular clusters often feature local anomalies in the size distribution of their ions that may be tentatively assigned to local anomalies in their stability. These so-called "magic numbers" in cluster size distributions have played a key role in the discovery of icosahedral arrangements in van-der Waals (vdW) bound clusters, rock-salt structures in alkali halide clusters, electronic shell structure of simple-metal clusters, and cage-like structures in fullerenes [156, 157, 161-165]. In all these systems, mass spectra suggested that certain cluster sizes were particularly stable, thus providing a clue to the geometric or electronic structure of the clusters. The relation between magic numbers and cluster stability is subtle; there is no one-toone relation between ion abundance $I_{N}$ and stability. For example, for fullerenes that can be ionized softly by near-threshold ionization, the exceedingly high abundance of $\mathrm{C}_{60}^{+}$observed in nozzle beams is a consequence of the high abundance of $\mathrm{C}_{60}$ which derives from growth kinetics rather than stability [166]. For alkali metal clusters the stability depends on the number of valence electrons rather than the number of atoms; hence the method chosen to ionize neutral precursors may affect the observed magic numbers [157].

Fortunately, for species considered in this Section (vdW or hydrogen bound clusters that are grown in neutral HNDs and subsequently ionized) the situation is rather straightforward: The growth process is statistical; the size distribution of neutral precursors (the HND as well as any embedded dopant clusters) will not feature any local anomalies. The formation of cations, on the other hand, is a violent process that releases $>10 \mathrm{eV}$, orders of magnitude more than the dissociation energy of 
the ions that are ultimately observed, $\mathrm{He}_{N} \mathrm{X}^{+}$where $\mathrm{X}$ is an atom, molecule, or cluster. Thus, the mass spectrometrically detected cluster ions will conform to the conditions of the evaporative ensemble: they have evaporated one or more monomers [66]. Whereas gross features of the size distribution (its envelope) will depend on the size distribution of neutral precursors and details of the ionization process, local anomalies in the size distribution of ions will signal the occurrence of local anomalies in their stability as measured by their dissociation (or evaporation) energy

$$
D_{N}=E_{N}-E_{N-1},
$$

where $E_{N}$ is the total binding energy (or atomization energy) of $\mathrm{He}_{N} \mathrm{X}^{+}$. Various suggestions have been made for the exact relation between $D_{N}$ and ion abundance $I_{N}$, see $[156,157,167,168]$ and references therein. Two often used relations are

$$
\begin{aligned}
\ln \left(I_{N+1} I_{N}^{-1}\right) & =\left(D_{N+1}-D_{N}\right) / k_{B} T \\
\ln \left(I_{N+1} I_{N-1} I_{N}^{-2}\right) & =\left(D_{N+1}-D_{N}\right) / k_{B} T,
\end{aligned}
$$

where $T$ is the vibrational temperature of the cluster ions $[169,170]$. The left-handsides (LHS) of eqs. (2) and (3) equal the first and second derivatives of $\ln I_{N}$, respectively. The difference $D_{N+1}-D_{N}$ on the right-hand side (RHS) equals the second derivative of the energy $E_{N}$. Eq. (2) was originally proposed to account for the thermal properties of the valence electrons in alkali metal clusters; it involved the second derivative of the free energy $F_{N}$ rather than $E_{N}[156,171]$. Although the local anomalies in the second derivative of $F_{N}$ become sharper in the limit $T \rightarrow 0$, i.e. if the entropy is neglected, no qualitative changes occur.

In Fig. 6 we test the proposed relations between cluster abundance and stability for $\mathrm{He}_{N} \mathrm{Ar}^{+}$. Panel (a) displays the ion abundance measured at our lab in Innsbruck, and the dissociation energy computed by Tramonto et al. using the PIMC method $[172,173]$. The graph was designed such that the data points for $I_{N}$ and $D_{N}$ coincide at $N=12$. $I_{N}$ and $D_{N}$ share the most prominent features, namely abrupt drops beyond $N=12,32,44$, followed by constant or gradually declining values. The PIMC study reveals that these magic numbers are related to the successive completion of three concentric solvation shells around $\mathrm{Ar}^{+}$, containing 12, 20, and $12 \mathrm{He}$ atoms, respectively. The centers of mass of the polymers that represent the He atoms in the solid-like shells are depicted in panel (a) of Fig. 6; the atoms are placed at the vertices of an icosahedron, dodecahedron, and icosahedron in the first, second and third shell, respectively [173].

Panel (b) of Fig. 6 compares the LHS and RHS of eq. (2). The thermal energy was set to $k_{B} T=15.6 \mathrm{meV}$ in order to bring the two expressions into agreement at $N=12$. Both expressions exhibit singular minima at the magic numbers, but their amplitudes in the RHS decrease much more quickly with increasing $N$ than in the LHS. Assuming a decrease of the temperature $T$ with increasing $N$ could possibly provide a remedy. Such a decrease would be reasonable because, as discussed below, larger, more weakly bound $\mathrm{He}_{N} \mathrm{Ar}^{+}$will tend to be colder. However, the approach is not practical if one attempts to estimate dissociation energies based on experimental abundance distributions. Furthermore, we will show that a thermal energy of $k_{B} T=15.6 \mathrm{meV}$ ( or $T=182 \mathrm{~K}$ ), postulated for $\mathrm{He}_{12} \mathrm{Ar}^{+}$, is unreasonably 


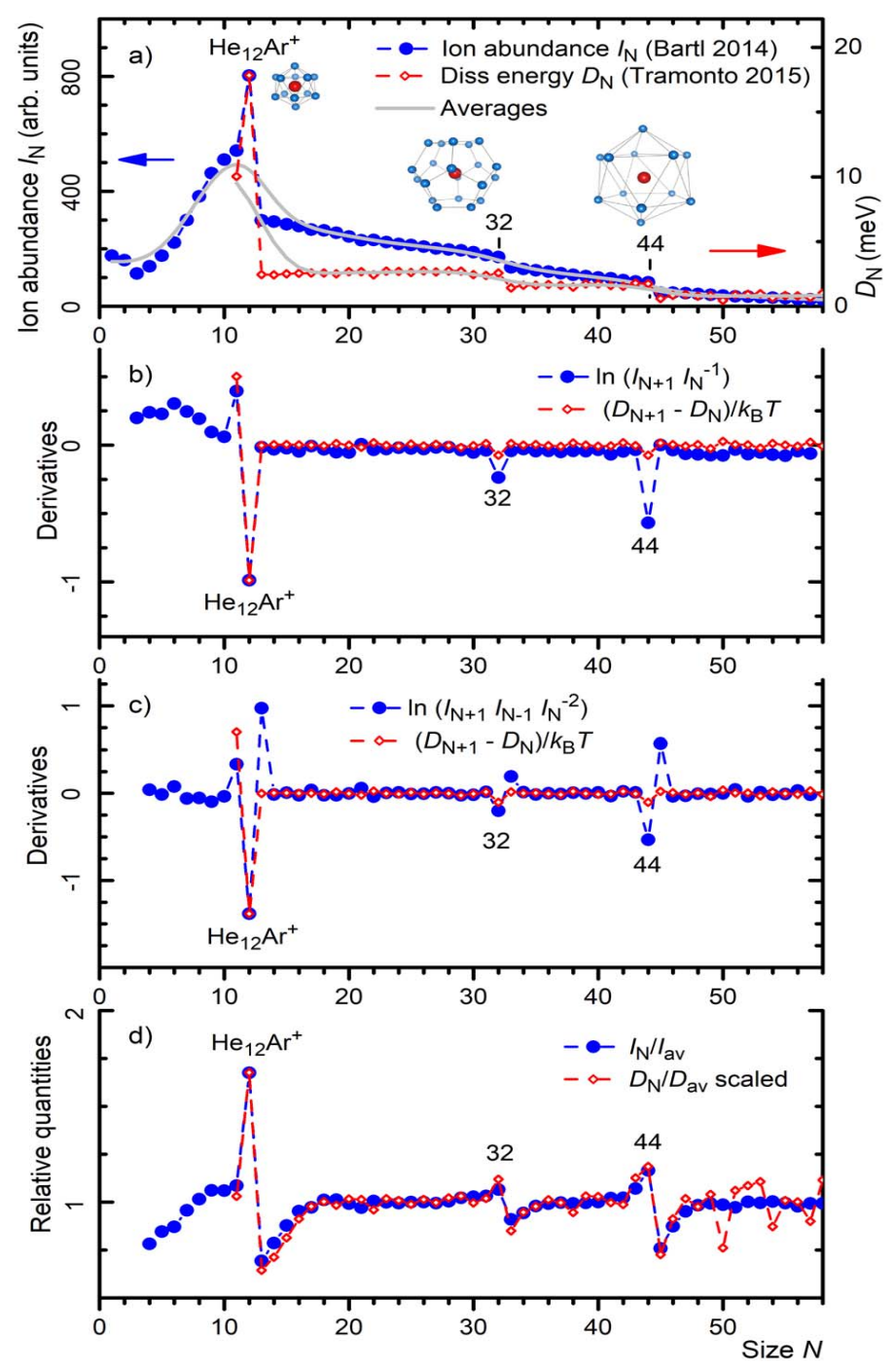

Figure 6. Panel (a): Comparison of measured ion abundances $I_{N}$ and computed dissociation (evaporation) energies $D_{N}$ of $\mathrm{He}_{N} \mathrm{Ar}^{+}$, reported by Bartl et al. and Tramonto et al. [172, 173]. Both quantities feature abrupt drops at $N=12,32$, and 44 which correlate with closure of three consecutive solvation shells of icosahedral symmetry. The computed time-averaged positions of He atoms in these shells are shown. Panels (b) through (d): Comparison of experimental abundances $I_{N}$ with computed dissociation energies $D_{N}$ as suggested by eqs. (2), (3), (4). The excellent agreement in panel (d) suggests that $I_{N}$ is approximately proportional to $D_{N}$.

high.

Fig. 6 (c) compares the LHS and RHS of eq. (3). The thermal energy was set to $k_{B} T=11.1 \mathrm{meV}(T=129 \mathrm{~K})$ in order to bring the two expressions into agreement at $N=12$. The agreement is poor; the satellite peaks in the second derivative of $I_{N}$ are not present in the first derivative of $D_{N}$.

Eqs. (2) and (3) have proven useful when data pertaining to charged metal clusters are analyzed. Why do they fail for $\mathrm{He}_{N} \mathrm{Ar}^{+}$? An important quantity in the discussion 
of evaporative ensembles is the heat capacity $C_{N}$ of an atomic (homonuclear) cluster ion $\mathrm{X}_{N}^{+}$. The classical (equipartition) relation $C_{N}=(3 N-7) k_{B}$ works reasonably well when $D_{N}$ is large and temperatures are in the classical regime [66]. The very weak binding of $\mathrm{He}_{N} \mathrm{X}^{+}$, however, implies low temperatures, the equipartition theorem will fail badly, and the heat capacity approaches zero. In this situation it has been argued $[122,124]$ that the following relation holds

$$
\frac{I_{N}}{I_{\mathrm{av}}}=\frac{D_{N}}{D_{\mathrm{av}}}
$$

where $I_{\mathrm{av}}$ and $D_{\mathrm{av}}$ are local (running) averages of $I_{N}$ and $D_{N}$; they describe the envelopes of these quantities.

Eq. (4) is tested in panel (d) of Fig. 6. Each data point in the local averages was computed by Gaussian weighting of its adjacent \pm 5 data points with a standard deviation of $\sigma=2$. A scaling factor was necessary in order to bring the LHS and RHS into agreement at $N=12$ : the "scaled" RHS expression plotted in Fig. 6 (d) equals $1+0.57\left(D_{N} / D_{\text {av }}-1\right)$. With that scaling (which reduces the amplitudes of local anomalies in the computed values of $D_{N}$ ) the agreement between the two curves becomes excellent. The shapes and amplitudes of the three anomalies match very well for all three magic numbers. (The RHS features additional anomalies beyond $N=44$ whose origin is unknown). As $I_{\mathrm{av}}$ and $D_{\mathrm{av}}$ are functions that do not change very quickly, we postulate that $I_{N}$ is approximately proportional to $D_{N}$ if one considers very weakly bound cluster ions.

We close with a brief discussion of the temperature of clusters in an evaporative ensemble. The concept is intricate; in his original model Klots introduced 3 different temperatures [66]. The concept of a microcanonical temperature, introduced by Andersen et al., provides a cleaner framework but its description is still beyond the scope of the current review $[67,174]$. Suffice it to say that the difficulties in defining a temperature disappear when the cluster is sufficiently large such that its drop in temperature $\Delta T$ upon evaporation of a monomer is negligible, i.e. $\Delta T \ll T$ or, in other words, when the cluster forms its own heat bath. A very useful relation for (neutral or charged) clusters forming an evaporative ensemble is

$$
D_{N} \approx \gamma k_{B} T
$$

where the so-called Gspann factor $\gamma$ is very nearly independent of cluster size $N$, and only weakly dependent on the time scale of the mass spectrometric time scale. A typical value is $\gamma \approx 25[66,174]$. As stated above, more weakly bound cluster ions will tend to be colder. For large clusters, $N \gg 10, D_{N}$ will approach the cohesive energy of the bulk. The cohesive energy of, say, argon, is $80 \mathrm{meV}$; the predicted temperature of neutral argon clusters in a beam, $37 \mathrm{~K}$, is in excellent agreement with values obtained by electron diffraction, see [175] and references therein.

For HNDs whose dissociation energy will be close to the bulk value $(0.616 \mathrm{meV}$, see [51]), eq. (5) predicts a temperature of $0.28 \mathrm{~K}$, in reasonable agreement with the measured value $0.37 \mathrm{~K}[56,65]$. We feel confident to apply eq. 5 for a prediction of the temperature of mid-sized $\mathrm{He}_{N} \mathrm{X}^{+}$if their dissociation energies are known. A typical value of $D_{N}=10 \mathrm{meV}$ would translate to $T=4.6 \mathrm{~K}$. The value of $D_{12}=17.8 \mathrm{meV}$ 
computed for $\mathrm{He}_{N} \mathrm{Ar}^{+}$would translate to $8.1 \mathrm{~K}$, an order of magnitude below the value that we had to use in our discussion of eqs. (2) and (3). This reinforces our conclusion that eqs. (2) and (3) should not be used for very weakly bound systems.

\subsection{The sizes of particularly stable $\mathrm{He}_{N} \mathrm{X}^{ \pm}$and $\left(\mathrm{H}_{2}\right)_{N} \mathrm{X}^{ \pm}$}

Having established a semi-quantitative correlation between measured abundances $I_{N}$ of $\mathrm{He}_{N} \mathrm{X}^{+}$and their dissociation energies $D_{N}$ we are now prepared to inspect mass spectral data for evidence for particularly stable sizes $N$. Magic numbers in mass spectra come in a variety of shapes but ledges, such as those observed for $\mathrm{He}_{32} \mathrm{Ar}^{+}$ and $\mathrm{He}_{44} \mathrm{Ar}^{+}$(Fig. 6 (a)) are quite common. Another frequently observed feature is a local maximum, such as that formed by $\mathrm{He}_{12} \mathrm{Ar}^{+}$in Fig. 6 (a). These anomalies may be less obvious if the envelope of the abundance distribution is rapidly changing with $N$ but eq. (4) provides a powerful criterion for visual inspection of mass spectra, even if one does not formally compute the average $I_{\mathrm{av}}$.

Tables 1 and 2 compile magic numbers reported for positively charged $\mathrm{He}_{N} \mathrm{X}^{+}$ where $\mathrm{X}$ is an atom, small molecule, or cluster. A few data pertain to dications $\mathrm{X}^{2+}$ or anions $\mathrm{X}^{-}$. The amplitude of the local anomaly may be large or small; the essential criterion is that it is significantly larger than the statistical fluctuation of $I_{N}$.

Also listed in Tables 1 and 2 are calculated values. These may refer to anomalies in computed dissociation energies, or to sizes at which radial distribution functions or other measures indicate closure of a solvation shell. Some theoretical studies have applied classical as well as quantum mechanical methods, sometimes with greatly different results [176]. For additional references to experimental and theoretical work see $[172,177-180]$ and references therein.

Some experimental entries in Tables 1 and 2 are non-integer. This happens, for example, if one observes a sigmoidal drop of the abundance rather than an abrupt drop.

The vast majority of experimental values listed in Tables 1 and 2 are derived from mass spectra, usually obtained by ionizing doped HNDs. Recently the Innsbruck group has pioneered an alternative approach, namely ionization and mass-selection of HNDs followed by doping (see section 3) [107]. This approach avoids the introduction of a large amount of excess energy into the cluster ion; it may be necessary to excite the cluster ions in a collision cell in order to strip excess helium and enhance the abundance of particularly stable ions [181].

Some entries in Tables 1 and 2 are obtained by other means, including measurements at high-pressure-low-temperature or in ion traps as discussed in Section 6. Also note that mass spectrometry offers more elaborate approaches that are better suited to measure the stability of cluster ions, such as measurements of kinetic energy release [182], metastable fractions [183], and dissociation of cluster ions in a heat bath [184] but these approaches have not yet been applied to $\mathrm{He}_{N} \mathrm{X}^{+}$.

Before highlighting a few persistent features or trends in Tables 1 and 2 it is useful to consult data obtained for the solvation of $\mathrm{X}^{+}$in molecular hydrogen. At low temperatures, the behavior of hydrogen clusters may be dominated by quantum delocalization and exchange effects; small clusters of para-hydrogen may be superfluid 
Table 1. The sizes $N$ of helium snowballs or other favored structures formed around ions $\mathrm{X}^{ \pm}$. Most experimental values mark the size at which the abundance of $\mathrm{He}_{N} \mathrm{X}^{ \pm}$exhibits a local maximum or stepwise decrease. Theoretical values refer to sizes for which computed dissociation energies display maxima or abrupt drops, or to the number of He atoms in a solvation shell which may depend slightly on the total number of He atoms in the simulated complex. Other theoretical values refer to subshell closures, closures of higher shells, or sizes at which a commensurate layer forms on a corrugated surface.

\begin{tabular}{|c|c|c|}
\hline$I^{ \pm}$ & Experiment & Theory \\
\hline $\mathrm{H}^{+}$ & 6, $13[186], 2,11[186]$ & $6[187]$ \\
\hline $\mathrm{H}_{3}^{+}$ & $10,11[118], 12$ [188] & \\
\hline $\mathrm{Li}^{+}$ & $2,6,8,14[189]$ & $\begin{array}{c}4,6,8[189], 8.2[48] \\
10[190,191]\end{array}$ \\
\hline $\mathrm{Li}_{2}^{+}$ & & $6,14,16,18[192]$ \\
\hline $\mathrm{Na}^{+}$ & $2,9[122]$ & 16 [193], 9 [194] \\
\hline & & $\begin{array}{c}12[48,195,196] \\
11-12[191]\end{array}$ \\
\hline & & $10,12[197,198]$ \\
\hline & & $3,5,8,9,12,16,18,19[199]$ \\
\hline $\mathrm{Na}_{2}^{+}$ & $2,6[122]$ & $2,6,20[192]$ \\
\hline $\mathrm{K}^{+}$ & $4[59], 2,12[122]$ & $\begin{array}{c}12[194], 15[195] \\
15[191,196]\end{array}$ \\
\hline $\mathrm{K}_{2}^{+}$ & & $6,12,17,24[192]$ \\
\hline $\mathrm{Rb}^{+}$ & $14[59]$ & $19.2[61]$ \\
\hline $\mathrm{Cs}^{+}$ & $12,16[59], 15[200]$ & $17.5[195], 21.4[61]$ \\
\hline & $17-18,50[139], 17$ [201] & 18 [196], 17 [201] \\
\hline $\mathrm{Cs}_{2}^{+}$ & $8[139]$ & \\
\hline $\mathrm{Cu}^{+}$ & $6,12,24[202]$ & \\
\hline $\mathrm{Cu}_{2}^{+}$ & $2[202]$ & \\
\hline $\mathrm{Ag}^{+}$ & $10,12,32,44[203], 12$ [181] & \\
\hline $\mathrm{Ag}_{2}^{+}$ & $6,12[181]$ & \\
\hline $\mathrm{Ag}^{2+}$ & $6,10,12[203]$ & \\
\hline $\mathrm{Au}^{+}$ & $12,14,32,44[126], 12[204]$ & $12,32,44[204]$ \\
\hline $\mathrm{Au}_{2}^{+}$ & $8,11,18,21[126]$ & \\
\hline $\mathrm{Be}^{+}$ & & $12[205], 15[195,196]$ \\
\hline $\mathrm{Mg}^{+}$ & $19-20[203]$ & 19 [195], 20 [206], 18 [196] \\
\hline $\mathrm{Mg}^{2+}$ & $4,8,10-11[203]$ & $9[206]$ \\
\hline $\mathrm{Zn}^{+}$ & $12[203]$ & \\
\hline $\mathrm{Cd}^{+}$ & $11 \pm 1[203]$ & \\
\hline $\mathrm{Pb}^{+}$ & $17[58]$ & 17 [207] \\
\hline $\mathrm{Pb}^{2+}$ & $12[58]$ & $12,15[207]$ \\
\hline
\end{tabular}


Table 2. Same as Table 1.

\begin{tabular}{|c|c|c|}
\hline Ion & Experiment & Theory \\
\hline $\mathrm{F}^{+}$ & $10.2 \pm 0.6[127]$ & \\
\hline $\mathrm{F}^{-}$ & $18.3 \pm 0.9[127]$ & $13.1 \pm 0.1[208]$ \\
\hline $\mathrm{Cl}^{+}$ & $11.6 \pm 0.2[127]$ & \\
\hline $\mathrm{Cl}^{-}$ & $19.5 \pm 0.2[127]$ & $17.3 \pm 0.3[208]$ \\
\hline $\mathrm{Br}^{+}$ & $13.5 \pm 0.1[127]$ & \\
\hline $\mathrm{Br}^{-}$ & $22.0 \pm 0.2[127]$ & $19.0 \pm 0.2[208]$ \\
\hline $\mathrm{I}^{+}$ & $16.3 \pm 0.9[127]$ & \\
\hline $\mathrm{I}^{-}$ & & $21.1 \pm 0.3[208]$ \\
\hline $\mathrm{I}_{2}^{+}$ & $20.0 \pm 0.1[127]$ & \\
\hline $\mathrm{Ne}^{+}$ & $11,13[119,180]$ & $7,10,12[209]$ \\
\hline $\mathrm{Ne}_{2}^{+}$ & $12,14[180]$ & \\
\hline $\mathrm{Ar}^{+}$ & $12[108,119,210]$ & $2,12,14[209]$ \\
\hline & $2,12,32,44[172]$ & $12,32,44[173]$ \\
\hline $\mathrm{Ar}_{2}^{+}$ & $7,12,14,17,20,26[172]$ & \\
\hline $\mathrm{Kr}^{+}$ & $10[211], 12[80,119,212]$ & \\
\hline & $12,14[172]$ & \\
\hline $\mathrm{Kr}_{2}^{+}$ & $12[212], 7,12,18,20[172]$ & \\
\hline $\mathrm{Kr}^{2+}$ & $12,32[213]$ & \\
\hline $\mathrm{Xe}^{+}$ & $4,8,15[211], 12[172]$ & \\
\hline $\mathrm{Xe}_{2}^{+}$ & $24[172]$ & \\
\hline $\mathrm{N}_{2}^{+}, \mathrm{O}_{2}^{+}, \mathrm{CO}^{+}$ & $12[120]$ & \\
\hline $\mathrm{H}_{2} \mathrm{O}^{+}$ & $12,32,44[181]$ & \\
\hline $\mathrm{CH}_{3} \mathrm{I}^{+}$ & $17.8 \pm 0.2[127]$ & \\
\hline $\mathrm{C}_{6} \mathrm{H}_{6}^{+}$ & & $34[176]$ \\
\hline $\mathrm{C}_{20} \mathrm{H}_{10}^{+}$corannulene & $32,45[214]$ & $4,7,12,17,32,45[214]$ \\
\hline $\mathrm{C}_{24} \mathrm{H}_{12}^{+}$coronene & $38,41,44[215]$ & $62[176], 44,50[216]$ \\
\hline$\left(\mathrm{C}_{24} \mathrm{H}_{12}\right)_{2}^{+}$ & $20[217]$ & \\
\hline $\mathrm{C}_{60}^{+}$ & $32,60[124]$ & $32,58[124], 32,60[218]$ \\
\hline $\mathrm{C}_{60}^{-}$ & $32,60[125]$ & \\
\hline $\mathrm{C}_{70}^{+}$ & $37,62[124]$ & \\
\hline $\mathrm{C}_{70}^{-}$ & $37,65[125]$ & \\
\hline
\end{tabular}

even if they show pronounced shell structures [11, 185].

Experimental values identifying particularly stable $\left(\mathrm{H}_{2}\right)_{N} \mathrm{X}^{+}$are listed in Table 3. They are mostly deduced from mass spectra which are either dominated by series of $\left(\mathrm{H}_{2}\right)_{N} \mathrm{X}^{+}$cluster ions or of hydrogenated cluster ions $\left(\mathrm{H}_{2}\right)_{N} \mathrm{HX}^{+}$. Hydrogenated species result from an exothermic reaction between $\mathrm{H}_{2}^{+}$and $\mathrm{H}_{2}$ that produces $\mathrm{H}_{3}^{+}+$ 
$\mathrm{H}$; the hyperthermal $\mathrm{H}$ may readily escape from the cluster ion [219]. However, the ion-molecule reaction will be suppressed if the ionization energy of $\mathrm{X}$ is well below that of $\mathrm{H}_{2}$, e.g., if $\mathrm{X}=\mathrm{C}_{60}[220,221]$. The charge will then be localized on $\mathrm{X}$ and non-hydrogenated ions $\left(\mathrm{H}_{2}\right)_{N} \mathrm{X}^{+}$will become prominent.

Table 3. The number $N$ of hydrogen molecules $\left(\mathrm{H}_{2}\right.$ or $\left.\mathrm{D}_{2}\right)$ that provides for particularly stable solvation of ions $\mathrm{X}^{ \pm}$. In some cases protonated ions $\left(\mathrm{H}_{2}\right)_{N} \mathrm{HX}^{+}$as well as non-protonated ions $\left(\mathrm{H}_{2}\right)_{N} \mathrm{X}^{+}$are observed; their magic numbers may differ slightly.

\begin{tabular}{ccc}
\hline Ion $\mathrm{X}^{ \pm}$ & Experiment & Theory \\
& & \\
$\mathrm{D}_{3}^{+}$ & $3,6,8,10[222]$ & \\
$\mathrm{H}_{6}^{+}$ & $12,54[219]$ & $12,32,44[224], 12[225]$ \\
$\mathrm{H}^{-}, \mathrm{D}^{-}$ & $12,32,44[223]$ & $6[227-229]$ \\
$\mathrm{Li}^{+}$ & $6[226]$ & $4[230]$ \\
$\mathrm{Li}_{3}^{+}$ & $8,12,32,44,52[200]$ & \\
$\mathrm{Cs}^{+}$ & $12,14,18[231]$ & \\
$\mathrm{Cu}^{+}$ & $4[232]$ & \\
$\mathrm{Cu}_{2}^{+}$ & $6[233]$ & \\
$\left.\mathrm{C}_{24} \mathrm{H}_{12}^{+} \mathrm{coron}_{2} \mathrm{D}_{2}\right)[231]$ \\
$\left(\mathrm{C}_{24} \mathrm{H}_{12}\right)_{2}^{+}$ & $32,36,38,53[217]$ & \\
$\left(\mathrm{C}_{24} \mathrm{H}_{12}\right)_{3}^{+}$ & $51,63[217]$ & \\
$\mathrm{C}_{60}^{+}$ & $61[217]$ & \\
$\left(\mathrm{C}_{60}\right)_{2}^{+}$ & $32,49[220,221] 32, \approx 49[234]$ & \\
$\mathrm{C}_{70}^{+}$ & $58[220,221]$ & \\
$\left(\mathrm{C}_{70}\right)_{2}^{+}$ & $37,51[220,221]$ & \\
& $68[220,221]$ & \\
\hline & & \\
\hline
\end{tabular}

Also included in Table 3 are theoretical results that pertain to the listed experimental systems. The search for efficient methods to store hydrogen at room temperature has stimulated theoretical work in the interaction of $\mathrm{H}_{2}$ with various metal ions; pertinent references may be found in recent reviews [235-238]. We note that the interaction between metal cations or halide anions and $\mathrm{H}_{2}$ is usually characterized as non-covalent [235-238], but theoretical work shows that the interaction in, e.g., $\left(\mathrm{H}_{2}\right)_{N} \mathrm{Cu}^{+}$is strongly covalent $[232,236]$.

The data in Tables 1, 2 and 3 reveal several intriguing features and trends; we will highlight a few. It is tempting to surmise the geometric structure of solvation shells from anomalies in the cluster ion abundance; the frequently observed magic number $N=12$, in particular, is often assigned to an icosahedral solvation shell. However, the discussion in Section 8 shows that this may be premature; quantum effects may preclude the formation of solid-like structures. For example, $\mathrm{He}_{12} \mathrm{~K}^{+}$forms a distinct magic number in mass spectra, but its computed structure factor suggests that its 
solvation shell is liquid-like [122, 239].

Data for alkali ions nicely demonstrate how the size of the solvation shell tends to increase as the size of the solvated ion increases. Interestingly, ions such as $\mathrm{Li}^{+}$that are "too small" to favor an icosahedral solvation shell often feature a sequence of smaller, ordered first solvation shells, as depicted in Fig. 11 for $N=4,6,8$ [189]. The sequence of halogen cations $\left(\mathrm{F}^{+}\right.$through $\left.\mathrm{I}^{+}\right)$also demonstrate the increase in the size of the solvation shell [127]. The same trend is observed for halide anions although, as expected for anions in general (see Section 2), the solvation shell is likely to remain liquid [127, 208]. Conversely, a mass spectrometric study of $\mathrm{Ag}^{+}$and $\mathrm{Au}^{+}$solvated in all noble gases (He through Xe) reveals how the number of atoms in the first solvation shell shrinks as the size of the solvent atoms increases [181, 204].

In Section 5.1 (Fig. 6) we already discussed the sequence of magic numbers $N=12$, 32, 44 observed for $\mathrm{He}_{N} \mathrm{Ar}^{+}$; the sequence suggests the formation of three concentric solvation shells of icosahedral symmetry $[172,173]$. Tanuma et al., based on their observation of magic $\mathrm{He}_{12} \mathrm{Kr}^{2+}$ and $\mathrm{He}_{32} \mathrm{Kr}^{2+}$, were probably the first to propose the existence of two solvation shells of $I_{h}$ symmetry [213]. Galli et al. proposed the existence of three solvation shells of $I_{h}$ symmetry, for $\mathrm{He}_{N} \mathrm{Na}^{+}$[196], but the expected magic numbers have not yet been reported for that system. Instead, the $N=12,32$, 44 sequence has been observed for several other systems, namely $\mathrm{Ag}^{+}, \mathrm{Au}^{+}, \mathrm{H}_{2} \mathrm{O}^{+}$ solvated in $\mathrm{He}, \mathrm{H}^{-}$or $\mathrm{Cs}^{+}$solvated in $\mathrm{H}_{2}$, and $\mathrm{D}^{-}$solvated in $\mathrm{D}_{2}$. The underlying structure is appealing because, in a classical picture, the solvent atoms or molecules in the second and third shell would reside at the centers of the polygons (so-called hollow sites) formed by the solvent atoms or molecules in the first and second shell, respectively. This may help to make the system more rigid: The stacking sequence in successive layers would be ABA. In contrast, in the more commonly encountered nested Mackay icosahedra shell closures would occur at $N=12,54,146, \ldots$ each shell would feature 20 triangular faces with atoms in a close-packed arrangement, and the stacking sequence would be that of an fcc crystal, ABCA [165].

Surprisingly, the sequence $N=12,32,44$ has never been reported for mass spectra of ions solvated in heavier noble gases (Ne through Xe), nor appeared in the numerous theoretical studies of metals ions solvated in the heavier noble gases, see [240, 241] and references therein. This difference has, to the best of our knowledge, not yet been explained. An important parameter that determines the size and structure of the first solvation shell is the equilibrium distance between the ion and a solvent atom relative to that between solvent atoms [181, 204, 242]. However, the variety of ions which induce nested solvation shells of $I_{h}$ symmetry for $\mathrm{He}, \mathrm{H}_{2}$ and $\mathrm{D}_{2}$ solvents indicates that the structure is forgiving. Calvo and Yurtsever concluded from PIMD simulations that the icosahedral multishell structure in $\left(\mathrm{H}_{2}\right)_{44} \mathrm{H}^{-}$is largely due to quantum effects that lower the atomic density and stabilize the outermost shell; the classical minimum energy structure exhibits a much lower symmetry [224]. On the other hand, a diffusion Monte Carlo (DMC) study led Kohno et al. to the conclusion that the ground state wavefunctions of $\mathrm{H}_{2}$ in $\left(\mathrm{H}_{2}\right)_{12} \mathrm{H}^{-}$are highly delocalized and do not resemble the icosahedral structure of the global energy minimum [225]. Moreover, the strong nuclear quantum effects result in a nearly complete orientational disordering of the $\mathrm{H}_{2}$ molecules. 
The preference of physisorbed atoms for hollow adsorption sites is well known. For the right choice of coverage and temperature, a layer of atoms physisorbed on a corrugated surface will form ordered structures that are commensurate with the symmetry of the substrate. Commensurate layers of He adsorbed on graphitic surfaces have been studied extensively, see [243-245] and references therein. On flat graphitic surfaces, though, the distance between adjacent hollow sites is smaller than the size of He; hence He will not form the $1 \times 1$ phase on graphite or graphene. The densest ordered phase will be the so-called $\sqrt{3} \times \sqrt{3}$ phase in which solvent atoms are located at second-nearest hollow sites. On curved graphitic surfaces, however, the distance between atoms adsorbed on hollow sites will increase. The atoms in $\mathrm{C}_{60}$ form 12 regular pentagons and 20 hexagons; mass spectra of $\mathrm{C}_{60}^{+}$solvated in $\mathrm{He}, \mathrm{H}_{2}$ or $\mathrm{D}_{2}$ do, in fact, feature an abrupt drop in the ion abundance at $N=32$ solvent particles [124, 220, 221]. The same anomaly is observed for $\mathrm{C}_{60}^{-}$anions in He [125]. $\mathrm{C}_{70}$ offers a total of 37 hollow sites and, sure enough, this magic number is observed for $\mathrm{C}_{70}^{+}$ solvated in $\mathrm{He}, \mathrm{H}_{2}$ or $\mathrm{D}_{2}$ or $\mathrm{C}_{70}^{-}$in $\mathrm{He}[124,125,220,221]$.

However, the commensurate $1 \times 1$ layer does not represent the highest possible coverage on fullerenes. Mass spectra of $\mathrm{He}_{N} \mathrm{C}_{60}^{ \pm}$suggest that the first solvation shell closes at $N=60$ (see Fig. 4 in Section 3 ) and that of $\left(\mathrm{H}_{2}\right)_{N} \mathrm{C}_{60}^{+}$at $49[124,125,221$ ], in agreement with theoretical work [124, 218, 234]. Much more detailed information about the properties of helium physisorbed on fullerenes has been obtained by optical spectroscopy as discussed in Section 7.

Polycyclic aromatic hydrocarbons (PAHs) form another class of molecules that offer corrugated surfaces. In Section 7 we will discuss a combined experimental and theoretical study of corannulene cations using spectroscopy. Here we discuss a mass spectrometric study of $\mathrm{He}$ and $\mathrm{H}_{2}$ adsorption on charged coronene $\left(\mathrm{C}_{24} \mathrm{H}_{12}\right.$, or Cor $)$ and its clusters [215, 217]. Electron ionization of HNDs doped with $\mathrm{H}_{2}$ and Cor produces $\left(\mathrm{H}_{2}\right)_{N} \mathrm{Cor}_{n}^{+}$and their hydrogenated counterparts $\left(\mathrm{H}_{2}\right)_{N} \mathrm{HCor}_{n}^{+}$. A mass spectrum and abundance distributions for $n=1,2$ are displayed in Fig. 7. Also shown is the distribution of $\mathrm{He}_{N} \mathrm{Cor}^{+}$recorded without $\mathrm{H}_{2}$ doping. Cor is a planar molecule. On either face, it offers 7 adsorption sites at its 7 carbon rings, plus 12 peripheral sites formed by the hydrogen atoms, or a total of 38 sites. A classical study of He adsorption on (neutral) Cor identifies, indeed, stepwise decreases in the adsorption energies at $N=14,26$, and 38 (the number 26 corresponds to a situation where every other peripheral site is occupied) [246]. Reality is more complex. Each of the three abundance distributions in Fig. 7 (b) features a triplet of closely spaced magic numbers; an anomaly at $N=38$ is seen for all ions. The origin of the other two magic numbers in each triplet is less straightforward to explain, but the shift of the triplet to smaller sizes, in going from $\mathrm{He}$ to $\mathrm{H}_{2}$, nicely correlates with the increasing size of the solvent [247]. A quantum mechanical study of $\mathrm{He}_{N} \mathrm{Cor}^{+}$by Calvo reproduces the high stability for $N=44$ but concludes that the solvation shell is not yet complete, not even at $N=50$ [216]. The author emphasizes that helium atoms adsorbed at the aromatic plane are highly fluxional.

Fig. 7 (c) shows a shift of magic numbers towards larger values for $\mathrm{H}_{2}$ adsorption on Cor ${ }_{2}^{+}$. Further shifts are observed for $\mathrm{Cor}_{3}^{+}$and $\mathrm{Cor}_{4}^{+}$; each additional Cor seems to offer approximately $11.4 \pm 0.6$ additional strongly bound adsorption sites [217]. In a 


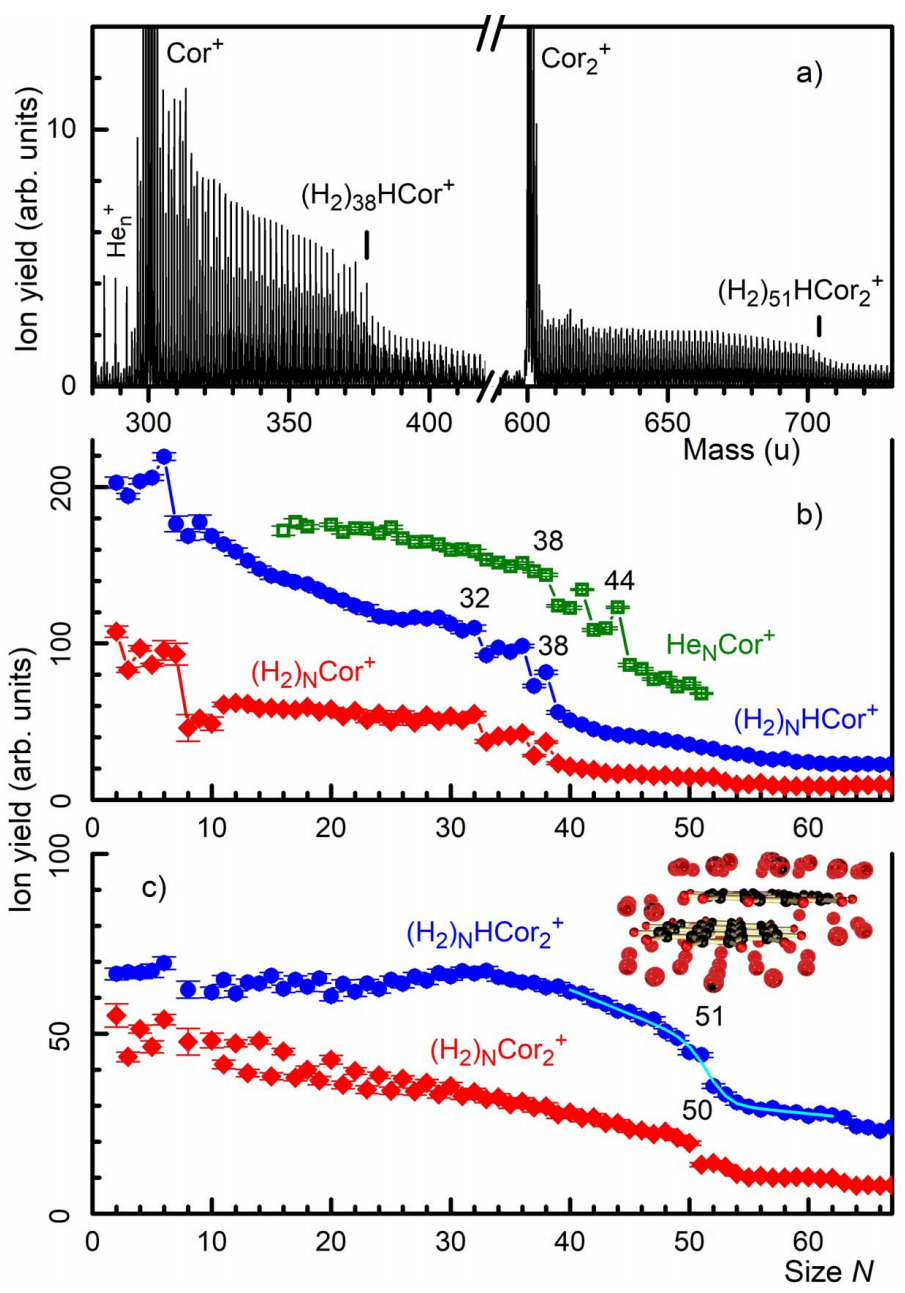

Figure 7. Panel a: Sections of a mass spectrum of HNDs doped with $\mathrm{H}_{2}$ and coronene $\left(\mathrm{Cor}, \mathrm{C}_{24} \mathrm{H}_{12}\right)$. Panel b: Abundance distributions of $\left(\mathrm{H}_{2}\right)_{N} \mathrm{HCor}^{+},\left(\mathrm{H}_{2}\right)_{N} \mathrm{Cor}^{+}$and $(\mathrm{He})_{N} \mathrm{Cor}^{+}$. Panel c: Abundance distributions of $\left(\mathrm{H}_{2}\right)_{N} \mathrm{HCor}_{2}^{+}$and $\left(\mathrm{H}_{2}\right)_{N} \mathrm{Cor}_{2}^{+}$. The cartoon illustrates the proposed structure of the coronene dimer ion, based on the observed shift in magic numbers.

cluster, the planar Cor molecules are expected to be stacked [248]. For a vertical stack, however, the number of strong adsorption sites, at the top and bottom of the stack, would not increase as more and more Cor molecules are added. The surface area of the mantle region would increase but adsorption energies in that region will be small. A possible explanation of the shift in the magic numbers is that adjacent molecules in the stack are shifted, thus forming terraces that offer additional adsorption sites, as illustrated in Fig. 7 (c) for the coronene dimer. A displacement of roughly $0.25 \mathrm{~nm}$ would account for the observed shift [217].

\subsection{Ion molecule reactions, fragmentation, anions, multiply charged ions}

In the mass spectra discussed so far, the ions were complexes containing $\mathrm{He}$ or $\mathrm{H}_{2}$. There are many instances though where the observed ion, formed upon electron 
ionization of doped HNDs, does not contain any He even though the HND played a key role in the formation of the observed ion. One example has already been discussed in Section 4 where a dopant atom or dopant cluster may move from the surface to an interior location upon ionization.

Here we will briefly discuss a few other topics where the HND has a profound effect even though no helium atoms are attached to the detected ion. In one way or another they are related to the effect of the HND on ionization-induced fragmentation. Early electron-ionization studies of HNDs doped with $\mathrm{Ar}, \mathrm{Kr}, \mathrm{Xe}, \mathrm{SF}_{6}$, and $\mathrm{H}_{2} \mathrm{O}$ seemed to suggest that clusters of these species could be softly ionized with negligible fragmentation of the dopant cluster; the helium atoms surrounding the nascent ion were supposed to rapidly quench fragmentation of the nascent ion [211]. However, later experiments have revealed that fragmentation of hydrogen-bound, vdW bound and metallic clusters upon electron ionization is substantial. The observed magic numbers are the same as those observed for bare clusters [80, 127, 151, 152, 249, 250]. Occasional differences may be caused by impurities. In particular, magic numbers assigned to clusters of rare gas $(\mathrm{RG})$ cluster ions $\mathrm{X}_{N}^{+}$may instead be due to protonated clusters $\mathrm{HX}_{N}^{+}$which can readily form in the presence of minor water contamination [186, 214, 251]. These species can have very different magic numbers, explaining some discrepancies between previous reports. Barring the misassignment of some mass peaks, observed magic numbers are the same for bare clusters and clusters embedded in HNDs. As the size distributions of the neutral precursors in HNDs are smooth, this implies that the cluster ions have lost at least one monomer [66]. A more quantitative assessment is possible if one analyzes intramolecular fragmentation. Here the results are mixed. Ellis and coworkers have investigated electron ionization of alcohols, ethers, and haloalkanes [57, 252]. The presence of helium did alter the fragmentation patterns when compared with the gas phase, with some ion product channels being more strongly affected than others. For some systems, relative parent ion intensities were enhanced by the helium. Cage effects seemed to favor hydrogen atom loss over other fragmentation channels.

Next we consider ion-molecule reactions in molecular clusters. $\mathrm{H}_{2} \mathrm{O}$ will serve as an example, but analogous results have been reported by the Innsbruck group for $\mathrm{H}_{2}$, $\mathrm{NH}_{3}, \mathrm{CH}_{4}, \mathrm{HCOOH}, \mathrm{CH}_{3} \mathrm{OH}, \mathrm{C}_{2} \mathrm{H}_{5} \mathrm{OH}$, and $\mathrm{C}_{3} \mathrm{H}_{4} \mathrm{~N}_{2}$ [219, 253-256]. The dominant ion series observed upon electron ionization of bare $\mathrm{H}_{2} \mathrm{O}$ clusters has the composition $\left(\mathrm{H}_{2} \mathrm{O}\right)_{N} \mathrm{H}^{+}$even though ionization of $\mathrm{H}_{2} \mathrm{O}$ monomers produces no $\mathrm{H}^{+}$[257]. The observed hydrogenated cations result from a proton transfer reaction between $\mathrm{H}_{2} \mathrm{O}^{+}$ and $\mathrm{H}_{2} \mathrm{O}$ producing $\mathrm{OH}$ and $\mathrm{H}_{3} \mathrm{O}^{+}$. The hyperthermal $\mathrm{OH}$ radical formed in the reaction escapes the cluster ion, hence no stoichiometric cluster ions $\left(\mathrm{H}_{2} \mathrm{O}\right)_{N}^{+}$can be detected if bare clusters are ionized. We have ionized HNDs doped with $\mathrm{X}=\mathrm{H}_{2} \mathrm{O}$ or $\mathrm{D}_{2} \mathrm{O}$ and observed stoichiometric ions $\mathrm{X}_{N}^{+}$with an ion yield of about $10 \%$ relative to that of $\left(\mathrm{H}_{2} \mathrm{O}\right)_{N} \mathrm{H}^{+}$[249]. In a related study of $\mathrm{H}_{2} \mathrm{O}$-doped HNDs, Ellis and coworkers observed a weak signal of $\left(\mathrm{H}_{2} \mathrm{O}\right)_{N}^{+}$as well as $\mathrm{He}\left(\mathrm{H}_{2} \mathrm{O}\right)_{N}^{+}$[258]. Apparently, the escape of the $\mathrm{OH}$ radical from the nascent water cluster ion can be quenched, although with a probability well below one, by the surrounding helium matrix.

If water-doped HNDs are co-doped with $\mathrm{C}_{60}$ the most prominent ion series above the mass of the fullerene is due to $\left(\mathrm{H}_{2} \mathrm{O}\right)_{N} \mathrm{C}_{60}^{+}$; the abundance of hydrogenated 
$\left(\mathrm{H}_{2} \mathrm{O}\right)_{N} \mathrm{HC}_{60}^{+}$is an order of magnitude weaker, at least for small sizes $N$ [259]. Another, weaker series of $\left(\mathrm{H}_{2} \mathrm{O}\right)_{N} \mathrm{OHC}_{60}^{+}$is detected as well. In this situation, the predominance of the stoichiometric $\left(\mathrm{H}_{2} \mathrm{O}\right)_{N} \mathrm{C}_{60}$ series is not owed to a quenching of the $\mathrm{OH}$ escape by the He matrix, but to the suppression of the ion-molecule reaction in the first place. The ionization energy of $\mathrm{C}_{60}$ is $7.6 \mathrm{eV}, 5.2 \mathrm{eV}$ below that of $\mathrm{H}_{2} \mathrm{O}$. Hence the charge will be localized on the fullerene; neutral $\mathrm{H}_{2} \mathrm{O}$ molecules will be adsorbed on the $\mathrm{C}_{60}^{+}$cation. This is a simplified view but ab-initio Hartree-Fock calculations show that the Mulliken charge on a $\mathrm{H}_{2} \mathrm{O}$ molecule remains below $0.05 e$ [259]. The Innsbruck group has reported analogous results for HNDs doped with $\mathrm{C}_{60}$ or $\mathrm{C}_{70}$ and $\mathrm{H}_{2}, \mathrm{NH}_{3}, \mathrm{CH}_{4}, \mathrm{HCOOH}, \mathrm{CH}_{3} \mathrm{OH}$ or $\mathrm{C}_{2} \mathrm{H}_{5} \mathrm{OH}[219,254,255,260,261]$.

In the example above, the HNDs served as a means to grow binary systems, but they had not direct impact on the processes following ionization of the dopant. We turn to a topic where the HND plays a more active role, namely the observation of homonuclear, multiply charged cluster ions $\mathrm{X}_{N}^{z+}$ with $z>1$. These ions are unstable (or metastable) with respect to charge separation if $N / z$ is small. In a mass spectrum, the abundance of $\mathrm{X}_{N}^{z+}$ usually features a well defined lower limit, the so-called appearance size $N_{a}$, which increases with charge state $z$ [262-264]. Theoretical studies of doubly charged alkali clusters have suggested that the appearance sizes are about twice as large as the Rayleigh limit $N_{\text {Ray }}$ at which the fission barrier vanishes. In other words, the highest fissility of the smallest observed $\mathrm{X}_{N}^{2+}, N_{\text {Ray }} / N_{a}$, is only about 0.5 . Several attempts to produce doubly charged alkali clusters with larger fissilities have failed. The likely reason is that the nascent doubly charged clusters are highly excited. They may cool by evaporation of monomers, but in doing so their fission barrier will gradually drop. Once the fission barrier drops below the evaporation energy, charge separation will occur [265].

For example, before making use of HNDs the smallest observed $\mathrm{Na}_{N}^{2+}$ contained $N_{a}=27 \mathrm{Na}$ atoms, in agreement with calculated appearance sizes (24 to 27). Calculated values for $N_{\text {Ray }}$ values, however, range from 6 to 12, see [266] and references therein. The Innsbruck group has doped HNDs with sodium and reported mass spectra that reveal dications as small as $\mathrm{Na}_{9}^{2+}$, indicating that dications with a fissility approaching 1 could be stabilized by HNDs. Similar results were reported for $\mathrm{K}_{N}^{2+}$, $\mathrm{Cs}_{N}^{2+}$, and $\mathrm{Cs}_{N}^{3+}$. Two factors may be responsible for the successful production of multiply charged alkali cluster ions that are much smaller than produced without help of a HND: First, the observed di- and trications may be the result of successive ionization which allows the cluster ion to relax and cool after each ionization event. Second, the HND may suppress fission. Although, as discussed above, a HND is not particularly effective in suppressing ionization induced fragmentation, fission is an unusually slow process. The slower the rate of fission the larger the chance that the helium matrix can remove the excess energy and quench the reaction.

A final topic that we will touch upon very briefly is electron attachment to doped HNDs. Electron attachment is a resonant process; each (fragment) anion produced upon attachment to a molecule $\mathrm{X}$ will feature one or more characteristic maxima in their ion yield measured versus the energy of the incident electron. The HND has two main effects, an energy shift in the resonances, and changes in the relative yield of the various resonances, for example due to suppression of intramolecular fragmentation. 
A blue shift of the resonances by about $1 \mathrm{eV}$ is to be expected because the bottom of the conduction band in helium lies above the vacuum level by approximately this amount [51]. The effect of the HND on the amplitude of resonances varies. As a rule of thumb, extensive and slow fragmentation processes are quenched effectively in the droplet, while direct dissociations via repulsive potential energy surface upon dissociative electron attachment cannot be suppressed [271].

Furthermore, it is known from gas-phase experiments on bare molecules that the temperature of the neutral precursor may impact the amplitude of a resonance [267]. Thus it is not surprising that the amplitude of resonances of molecules embedded in a HND may differ significantly from those observed for molecules vaporized from a hot source [267].

At elevated electron energies helium atoms may be excited and subsequently bind the incident electron. These metastable atomic He anions may contribute to the yield of dopant anions, and the formation of cations or dianions [100, 268-270]. For additional references the reader is referred to recent reviews [26, 271].

\section{Other experimental work}

A number of alternative methods to doping helium droplets have also been utilized to produce ions embedded in He atoms. To do this they all have to overcome the same obstacles regarding the low binding energy of helium adatoms, which requires cooling the ions to low temperatures while they interact with the He gas. In the late 1980s a group at the Tokyo Metropolitan University developed a high pressure drift tube to produce He cluster cations [117]. The apparatus consisted of a $10 \mathrm{~cm}$ long drift tube with a diameter also of $10 \mathrm{~cm}$ that was cooled with liquid helium to $4.4 \mathrm{~K}$ which contained a He buffer gas with a pressure in the range of 0.01-0.06 Torr, corresponding to a particle density of $2.2-13.2 \times 10^{16} \mathrm{~cm}^{-3}$. Positively charged clusters of He containing up to 15 atoms were formed by passing $\mathrm{He}^{+}$ions into the tube with a kinetic energy of $20 \mathrm{eV}$ and driving them forward with static electric field. The ions quickly thermalized with the buffer gas and acted as nucleation site for cluster growth. By varying the drift voltage, the authors were able to determine the evaporation energy as a function of cluster size, identifying solvation shell closures by sharp steps in the binding energies. Their identified shell closures were consistent with magic numbers identified in previous studies of He clusters grown in the supersonic expansion of neutral gas that were subsequently ionized [117, 272].

A few years later the same group used the same drift tube technique to solvate other atomic and molecular ions in helium $[118,119]$. Clusters of the form $\mathrm{RgHe}_{N}^{+}$, with $\mathrm{Rg}=\mathrm{Ne}, \mathrm{Ar}$, and $\mathrm{Kr}$, and with $N \leq 14$, were produced in much the same way as the pure $\mathrm{He}_{N}^{+}$clusters but by injecting ions of the heavier rare gases into the drift tube. Reactions of the type $\mathrm{RgHe}_{N-1}^{+}+\mathrm{He}+\mathrm{He} \rightarrow \mathrm{RgHe}_{N}^{+}+\mathrm{He}$, three-body (3B) processes where excess energy was carried away by one of the interacting He atoms, facilitated by the high pressure in the tube, were determined be responsible for the cluster growth. They identified that the mixed formed icosahedral structures with the charge centers of the clusters consisting of covalent $\mathrm{NeHe}^{+}$or isolated $\mathrm{Ar}^{+}$or $\mathrm{Kr}^{+}$ ions, respectively [119]. In other measurements, the authors also managed to solvate 


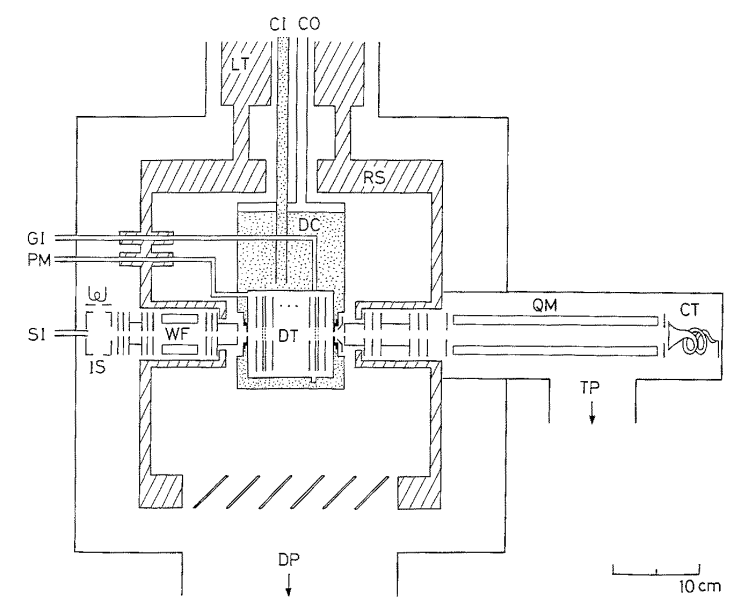

Figure 8. Schematic of setup used at the Tokyo Metropolitan University to embed ions in He using a high pressure drift tube (DT) [119]. Reprinted by permission from Springer Nature [119].

hydrogen cations with $\mathrm{He}$ [118]. Injecting $\mathrm{H}_{2}^{+}$ions in drift tube, they observed $\mathrm{HHe}_{N}^{+}$ $(N \leq 14)$ clusters as the sole cluster type. However, when seeding the drift tube with $\mathrm{H}_{3}^{+}$ions, they observed strong signals from $\mathrm{HHe}_{N}^{+}$and $\mathrm{H}_{3} \mathrm{He}_{N}^{+}$, as well as a weaker contribution from $\mathrm{H}_{2} \mathrm{He}_{N}^{+}(3 \leq N \leq 10)$ clusters. The method of producing Hesolvated ions in cooled, high pressure drift tubes was in subsequent years successfully applied to other small molecular systems like $\mathrm{N}_{2}^{+}, \mathrm{O}_{2}^{+}$, and $\mathrm{CO}^{+}[120,273,274]$.

An alternative approach developed at Yamanashi University in Japan used a pulsed electron beam mass spectrometer with the ion source mounted to a cryocooler $[222$, 275]. Rare gases were introduced into the ion source under relatively high pressures (on the order of a few Torr) and ionized by electron impact. A variable delay was implemented before the extraction of the ions which allowed the time-dependent study of cluster growth reactions of the form $\mathrm{Rg}_{N-1}^{+}+2 \mathrm{Rg}=\mathrm{Rg}_{N}^{+}+\mathrm{Rg}$, where $\mathrm{Rg}=\mathrm{He}, \mathrm{Ne}, \mathrm{Ar}, \mathrm{Kr}$, or Xe. The authors found a distinctive transition in binding energies between $N=3$ and 4 , indicating that a tightly bound trimer forms the ionic core in all of the cationic Rg clusters [275].

Neutral, loosely bound complexes of $\mathrm{Rg}$ and other atomic and molecular species have also been produced in the supersonic expansion of seeded Rg, with such studies going back several decades [276]. One of the earliest reports of ions solvated in $\mathrm{He}$ using this technique came from a study of hexafluorobenzene $\left(\mathrm{C}_{6} \mathrm{~F}_{6}^{+}\right)$co-expanded with a helium carrier gas by Terry Miller and coworkers [277]. Multi-photon ionization of the clusters resulted in ionic complexes containing up to three He atoms that were studied using laser-induced fluorescence spectroscopy [277]. A similar technique was also used by the group of John Maier in their pursuit after methods for measuring absorption spectra from ions that induce weaker chemical shifts to band positions than, e.g., Ne or Ar matrix isolation spectroscopy. Early progress was made in the 1990s when the group succeeded in producing $\mathrm{N}_{2}^{+}-$He complexes in a device that combined a supersonic jet of a $\mathrm{N}_{2} / \mathrm{He}$ mixture with an an electron impact ion source [278]. The resulting ions had a temperature of about $50 \mathrm{~K}$, which was cold enough for the He-tagged ions to be formed. The same approach was later used to produce other small molecular ions complexed with single $\mathrm{He}$ atoms, such as $\mathrm{HCO}^{+}$and $\mathrm{N}_{2} \mathrm{H}^{+}$, for 
spectroscopic studies [279-284].

Seeded supersonic jets were, however, insufficient for cooling more complex ions to low enough temperatures to allow for He solvation to take place. This required longer timescales and more active methods of cooling. An approach that allowed for this was the capturing of ions in a cooled, radio-frequency (RF) ion-trap, a technique developed in Berlin by Knut Asmis and coworkers [285]. This allowed for the formation of small polyatomic cluster ions that could be tagged with He atoms and they used this technique to measure the vibrational spectra of $\mathrm{VO}^{+}, \mathrm{VO}_{2}^{+}$, and $\mathrm{VO}_{3}^{+}$[285]. The Maier group later used this type of technique to successfully produce large, Hetagged molecular ions, such as $\mathrm{C}_{60}^{+}$and polycyclic aromatic hydrocarbon cations, in ion traps [114, 286-290]. Confining the ions in a 22-pole RF ion trap [291, 292], they injected a He buffer gas with temperatures as low as $3 \mathrm{~K}$. Through interactions with the cold gas, the ions rapidly cooled to thermal equilibrium with both vibrational and rotational degrees of freedom. At temperatures in the range of 3-4 K, the ions could be sufficiently cooled to allow for the formation of ion-He complexes. This was achieved for a range of different complex hydrocarbon species, most notably fullerene cations, to which up to 4 He atoms could be attached for use in action spectroscopy measurements. Further details on the spectroscopy of He-solvated ions is covered in Section 7 .

\section{Applications in spectroscopy}

Ionic species solvated in He find widespread use in spectroscopic applications. Reactive ionic species and species for which high vapor pressures are difficult to obtain, particularly at low temperatures, present challenges for conventional spectroscopic techniques where light is passed through a cell containing the studied substance and absorption or emission is measured directly. An effective method for obtaining the absorption spectra from dilute ionic species is matrix isolation spectroscopy [293-295]. In this method, ions are deposited into an inert matrix of a solid noble gas, typically Ne or Ar, which is cooled to temperatures as low as $5 \mathrm{~K}$. This cold environment freezes out vibrational and rotational degrees of freedom of the embedded ions, allowing for the measurement of a close approximation of their gas phase electronic spectra. However, even with the relatively weak interactions between the matrix and the deposited ion significant band shifts and broadening are often still observed. As an example, early measurements of the $\mathrm{C}_{60}^{+}$absorption spectrum from ions in Ne and Ar matrices [296] identified a number of bands that were later shown to tentatively match a pair of diffuse interstellar bands [297], ubiquitous absorption features assumed to originate from large molecules in the interstellar medium [298, 299]. However, the limited resolution and shifted positions of the measured $\mathrm{C}_{60}^{+}$bands were insufficient for a conclusive match with the observations. With its even weaker interaction strength, matrix isolation using He could potentially offer numerous improvements over $\mathrm{Ne}$ or Ar matrices. However, the high mobility of atomic and molecular species in He prevent matrix isolation with bulk He from being practical [21]. Instead, He droplets and clusters containing solvated neutral and ionic species can be utilized for spectroscopic applications [21, 300-303]. 
A particularly powerful method for ion spectroscopy is messenger spectroscopy, a type of action spectroscopy developed in the middle of the 1980s [304]. In messenger spectroscopy, ions are tagged with weakly bound "messenger" atoms or molecules and probed with a laser. If the tagged ion absorbs photons, i.e. if the laser wavelength matches a resonance in the system, the excitation energy will heat the system and cause one or more of the weakly bound messengers to be lost, which can be measured using a mass spectrometer [304]. The method has been established as a widely used technique in ion spectroscopy [27, 30, 31, 301-303, 305]. Helium atoms are optimal messengers because they interact very weakly with most neutral and ionic species and will thus induce a relatively weak perturbation to the tagged species being studied. This has been one of the main driving forces for producing ionic species solvated in He leading to the development of new techniques covered in Section 6.

A standout result using this He tagging messenger spectroscopy in recent years was the confirmation of $\mathrm{C}_{60}^{+}$as a carrier of at least five diffuse interstellar bands (DIBs), the first solid identification of any DIBs by the Maier group [114, 286]. Producing He-tagged fullerene ions in an ion trap, they were able to measure the depletion of tagged ions as a function of wavelength of a scannable laser. The resulting spectrum had a resolution comparable to astronomical observations, significantly better that their previous measurements using matrix isolation techniques [296], and only a small offset in the band positions due to the presence of the He tag $[114,286]$. Shortly after this, their results were confirmed by measurements at the University of Innsbruck where $\mathrm{C}_{60}^{+}$ions solvated in He were produced from He nanodroplets [123]. In these measurements ions solvated with up to at least $100 \mathrm{He}$ atoms were observed and the action spectra were recorded both as a function of wavelength and the number of solvating He atoms [123]. They showed (see Figure 9) that the band positions were redshifted with constant $0.72 \mathrm{~A}$ per He atom for the first $32 \mathrm{He}$ atoms [123]. Subsequent solvation up to a total of $60 \mathrm{He}$ atoms resulted in a slight blueshift compared to the band positions with 32 atoms, after which the band positions were again redshifted while they asymptotically approached constant values as the solvation approached bulk conditions [123]. The constant redshift induced by the first 32 He atoms was explained by these tags occupying localized positions above the 32 faces of the spherically symmetric fullerene cation. The next $28 \mathrm{He}$ atoms (from 33 to 60 in total) partially displaced the first, localized atoms leading to a process similar to a phase transition where the average interaction strength per He with the fullerene was weakened, leading to the blueshift of band positions in this regime. The first solvation monolayer was completed when $60 \mathrm{He}$ atoms solvated the ion, and additional atoms began forming another solvation layer outside of the first one with an overall weaker influence on the $\mathrm{C}_{60}^{+}$than the first layer. This interpretation agreed very well with previous studies of $\mathrm{C}_{60}^{+}$solvated in $\mathrm{He}$ [124] and with followup measurements by the Maier group with ions tagged with up to three He atoms [287].

Later spectroscopic measurements of $\mathrm{He}_{N} \mathrm{C}_{60}^{+}$complexes showed that not only did the band positions change as a function of the degree of solvation, but the widths of certain bands changed too [306]. The 32 faces of the $\mathrm{C}_{60}^{+}$ion consist of 12 pentagonal and 20 hexagonal rings, and the binding energy of a He atom to a hexagon is slightly stronger (by about $2 \mathrm{meV}$ ) than to a pentagon. Additionally, the difference in binding 


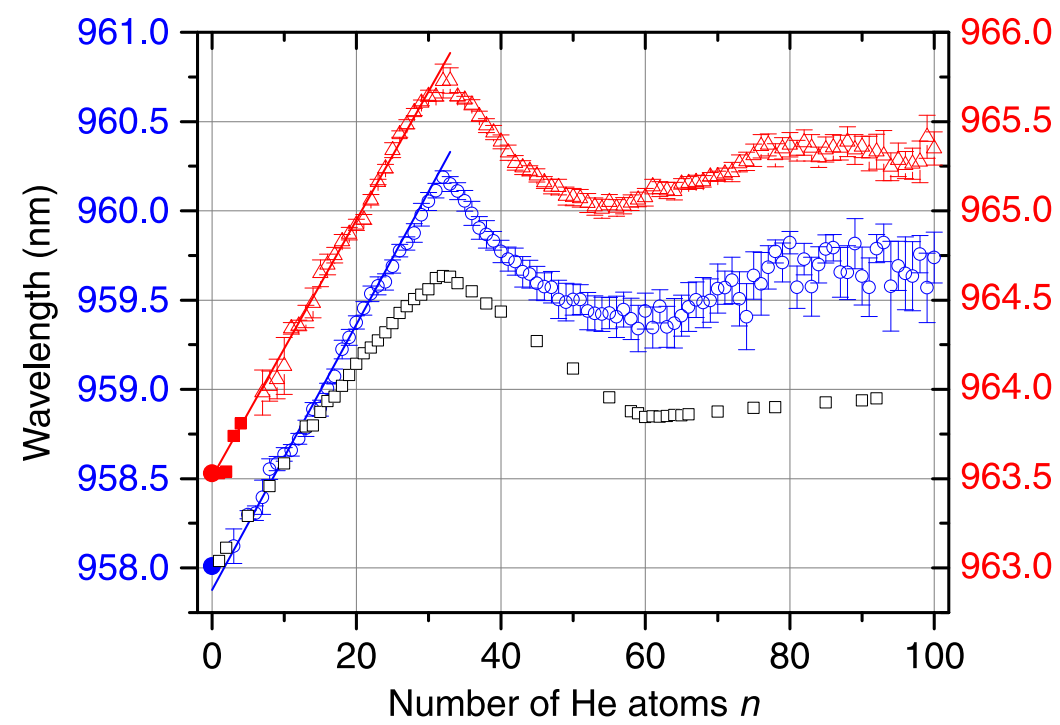

Figure 9. Center positions of the $963.5 \mathrm{~nm}$ (red) and $957.9 \mathrm{~nm}$ (blue) bands of $\mathrm{He}_{N} \mathrm{C}_{60}^{+}$as a function of the number of solvating He atoms, $N$. The open circles and triangles are by Kuhn et al. [123] and the solid points are from measurements by the Maier group [114, 286, 287]. The open squares are predictions from a jellium polarization model [123].

energies between the two types of sites changes somewhat between the electronic ground state and the first excited state of the solvated fullerene cation [306]. This change in binding energy results in the $963.5 \mathrm{~nm}$ band, but not the similarly bright 957.9 band, displaying slight shifts depending on the position of the He tags. For a mixture of isomers, the slightly different band positions led to a perceived broadening of the band. The width of this band was broadest for ions tagged with 16 He atoms, and narrowest for systems with 1 or 32 He atoms [306]. This was explained using combinatorics and by sampling the number of available isomers for different numbers of solvating He using PIMD over a range of temperatures [306]. The number of possible isomers, where each He atom occupied one of the 32 faces of $\mathrm{C}_{60}^{+}$, is the largest for $n=16$ (about $6 \times 10^{8}$ isomers) and the smallest for $n=1$ or 32 (2 possible isomers each) and the widths of the band were the result of the unresolved superposition of narrow bands from all of the available isomers, each with slightly different positions [306].

Additional studies have searched for other DIBs carriers using the helium messenger technique. Polycyclic Aromatic Hydrocarbon (PAH) cations are considered good candidates for this based on their expected ubiquity in the interstellar medium [307]. However, the absorption spectra for the cationic coronene and corannulene, two highly symmetric and compact (pericondensed) PAH species have revealed no matches with any known DIBs [214, 290]. Compared to the spectra of the spherically symmetric He-solvated $\mathrm{C}_{60}^{+}$, the band positions from the solvated, bowl-shaped corannulene cations displayed an erratic behavior as a function of the degree of solvation. The maximum redshift was coincidently once again observed for $32 \mathrm{He}$ atoms, but a number of anomalies were observed below this value [214]. These were explained using PIMD simulations of the He solvated corannulene cation to be caused by par- 
tial solvation shell closures with increasing degree of solvation. Like for $\mathrm{C}_{60}^{+}, 32 \mathrm{He}$ atoms represented the closure of a largely localized first layer. This layer also underwent a phase transition with the addition of more He atoms until the first monolayer was completely filled by 45 adatoms [214]. Most of the features attributed to partial shell closures were not visible in the mass spectrum, but only in the action spectrum [214]. This highlighted the sensitivity of He messenger spectroscopy in identifying the geometrical structure of solvated species and this method could be used on other systems, such as metal nanoparticles, to determine their structures.

\section{The theoretical description}

From the theoretical side, different strategies have been employed in order to investigate the solvation structures formed by helium around alkali metal cations. On the one hand, the interaction between the impurity and the He atoms has been described by means of a vast number of techniques ranging from $a b$ initio calculations to analytical model potentials. On the other hand, there is a number of numerical approaches used in the analysis of such doped helium droplets in the existing literature. In this section we review both crucial aspects of the theoretical investigation of these clusters.

\subsection{Interactions}

A fundamental step to obtain the corresponding intermolecular interactions with high accuracy requires the use of first-principles electronic structure calculations. In general terms, although there are many advances in procedures in which electron and nuclei are treated together in many different ways [308, 309], the separation of their motions is still predominant, in what is generally known as Born-Oppenheimer approximation. In this more prevalent treatment, the so-called super- molecular approach is in general used for the smallest cluster, while for the intermediate and large complex sizes the application of force fields is more convenient, provided that they are previously validated on benchmark first-principles calculations. The advantage of the latter is two-fold, since all cluster sizes are treated on the same footing and level of theory allowing in principle the study of their structure and dynamics.

A series of $a b$ initio studies have focussed on the calculation of the interaction between rare gas $(\mathrm{Rg})$ atoms and monocations $\mathrm{X}^{+}$in small size $\mathrm{Rg}_{N} \mathrm{X}^{+}$clusters [193, 310-318]. For larger systems, force fields and model potentials are required. These latter options usually involve the estimation and modeling of two different main contributions: on the one hand, long range attractive terms due to induction and dispersion forces with a dependence on the distance $R$ between $\mathrm{X}^{+}$and the Rg atom which ranges between $R^{-4}$ and $R^{-8}$, and, on the other hand, short range exchange-repulsion terms. Both contributions can be determined and adjusted by exploiting physical quantities such as binding energies, equilibrium distances and fundamental vibration frequencies [319], which can be obtained through experimental data or $a b$ initio estimations. The interactions which hold these atomic clusters together, also named vdW or long-range forces, are intrinsically noncovalent and their representation is different from that required for a regular molecule composed of a 
Table 4. Values of the parameters in Eqs. (7) and (8) for the 2B interactions in the $\mathrm{He}_{N} \mathrm{Li}^{+}$and $\mathrm{He}_{N} \mathrm{Cs}^{+}$systems studied in Refs. [189, 201].

\section{$\mathrm{He}-\mathrm{Li}^{+} \mathrm{He}-\mathrm{Cs}^{+} \quad \mathrm{He}-\mathrm{He}$}

$\begin{array}{cccc}R_{m}(\AA) & 1.90 & 3.35 & 2.97 \\ \epsilon(\mathrm{meV}) & 81.30 & 13.70 & 0.947 \\ \beta(\mathrm{meV}) & 4.2 & 9.5 & 8.0 \\ m & 4 & 4 & 6\end{array}$

similar number of atoms.

The simplest description for these weak interactions in a $\mathrm{He}_{N} \mathrm{X}^{+}$cluster reduces the general expression [320,321] based on a many-body expansion of the total potential to the pairwise additive two-body (2B) atom-atom term:

$$
V_{\text {tot }}\left(\mathbf{R}_{1}, \cdots \mathbf{R}_{N}, \mathbf{R}_{\mathrm{X}^{+}}\right)=\sum_{i<j}^{N} V_{\mathrm{He}-\mathrm{He}}\left(\left|\mathbf{R}_{i}-\mathbf{R}_{j}\right|\right)+\sum_{i=1}^{N} V_{\mathrm{X}^{+-} \mathrm{He}}\left(\left|\mathbf{R}_{i}-\mathbf{R}_{\mathrm{X}^{+}}\right|\right),
$$

where the potential in Eq. (6) depends on all the internal distances between the constituent atoms.

Different analytical expressions of this potential with parameters fitted to either $a b$ initio results or to experimental data have been commonly employed in the literature. Simple Lennard-Jones type potentials or more sophisticated options such as semi-empirical methods [322-324] adapted to describe the existing interactions in molecular clusters formed by He atoms and ions [325-328] are suitable options. A possible choice, successfully used for systems such as $\mathrm{He}_{N} \mathrm{Li}^{+}$[189] or $\mathrm{He}_{N} \mathrm{Cs}^{+}$[201], is the so-called improved Lennard-Jones (ILJ) potential [329], expressed as:

$$
V(r)=\varepsilon\left[\frac{m}{n(r)-m}\left(\frac{R_{m}}{r}\right)^{n(r)}-\frac{n(r)}{n(r)-m}\left(\frac{R_{m}}{r}\right)^{m}\right]
$$

where $\varepsilon$ and $R_{m}$ correspond to the potential depth well and the equilibrium distance, respectively, and

$$
n(r)=\beta+4\left(r / R_{m}\right)^{2} .
$$

In the above Eq. (8), $\beta$ is an adimensional parameter which modulates the stiffness of the potential curve and $m$ is equal to 6 or 4 for neutral-neutral and ion-neutral interactions, respectively. The procedure followed to obtain the corresponding parameters starts with an initial guess of sensible values considering some molecular properties such as polarizabilities of the involved partners.

In a second step this set of parameters is refined by comparison with high level $a b$ initio calculations. An example of the application of such a procedure for $\mathrm{He}_{N} \mathrm{Cs}^{+}$ 
can be found in Ref. [201]. Coupled-cluster calculations with single, double and perturbative triple excitations $[\mathrm{CCSD}(\mathrm{T})]$ together with the d-aug-cc-pV6Z and def2AQVZPP basis sets for $\mathrm{He}$ and $\mathrm{Cs}^{+}$, respectively, allowed to obtain interaction energies sufficiently accurate to give a deviation less than $0.3 \mathrm{meV}$ in the minimum region with respect to estimations carried out with the d-aug-cc-pV5Z/def2-AQVZPP set. For the case of $\mathrm{He}_{N} \mathrm{Li}^{+}$clusters, a similar study [189] based on CCSD(T) computations and exploiting a complete basis set limit for the interaction energies was also carried out. Optimized values for the parameters employed in the ILJ representation of Eqs. (7) and (8) of the interparticle interactions for the $\mathrm{He}_{N} \mathrm{Cs}^{+}$and $\mathrm{He}_{N} \mathrm{Li}^{+}$ clusters are shown in Table 4.

Figure 10 shows the potential for the $\mathrm{He}-\mathrm{He}, \mathrm{He}-\mathrm{Cs}^{+}$and $\mathrm{He}^{-\mathrm{Li}^{+}}$interactions obtained as explained above. The comparison of these potential curves reveals that such interactions correspond indeed to extreme cases where both potential depths and equilibrium distances are quite different. The intrinsic nature of the existing interactions in these doped helium droplets leads to a significant effect on physical properties such as structures and energetics.

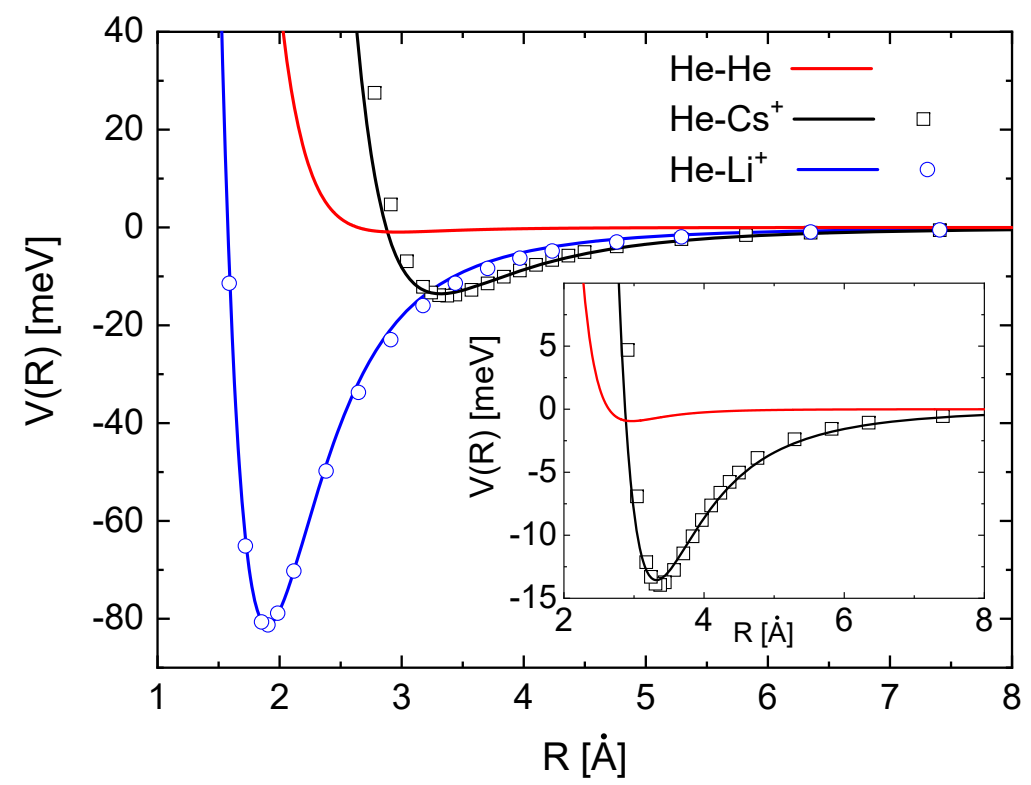

Figure 10. Potential energy curves for the He-He, $\mathrm{He}-\mathrm{Li}^{+}$and $\mathrm{He}-\mathrm{Cs}^{+}$interactions. Open symbols correspond to ab initio results while solid lines are for ILJ force fields. Inset shows an expanded view. Figure adapted from Ref. [201].

The pairwise description shown in Eq. (6) is affected by certain limitations in the case of vdW interactions [330, 331]. In fact, since inaccuracies committed with additive interactions increase with the cluster size, such approach is generally restricted to droplets with a small number of He atoms. Many-body contributions related to the 
dispersion, exchange and charge-induced dipole components of the total interaction between the ion and the helium atoms can play a nonnegligible role [35]. The necessity of a proper representation of all these contributions has then been the subject of discussion in investigations of charged systems [317, 331-335]. In some cases, the effect of 3B terms is found to be rather small. Thus, for example, Marinetti et al. only found some shifts to larger distances of radial distributions for $\mathrm{He}_{N} \mathrm{Li}^{+}$clusters [336] and Sebastianelli et al. concluded that the overall interactions obtained by means of $a b$ initio calculations for the smallest sizes, $N \leq 6$, are in fact governed by diatom-like interactions between the $\mathrm{Li}^{+}$ion and the He atom [317]. In turn, for $\mathrm{He}_{N} \mathrm{Na}^{+}$, energies predicted via a $2 \mathrm{~B}$ approximation for large clusters were found to be overestimated in comparison with those calculated when a self consistent manybody contribution between induced dipoles was added to the purely pairwise diatomic energy curves [197].

The effect of many body contributions was analysed for the case of $\mathrm{He}_{N} \mathrm{Li}^{+}$[189] by adding a 3B induced dipole-induced dipole term to the total potential as follows:

$$
\begin{aligned}
V_{3 \mathrm{~B}}\left(r_{i}, r_{j}, r_{i j}\right)= & -\frac{\alpha^{2}}{4}\left[3 r_{i} g_{3}\left(r_{j}\right) g_{5}\left(r_{i j}\right)+3 r_{j} g_{3}\left(r_{i}\right) g_{5}\left(r_{i j}\right)\right. \\
& -g_{3}\left(r_{i}\right) g_{3}\left(r_{j}\right) g_{1}\left(r_{i j}\right)-6 g_{1}\left(r_{i}\right) g_{1}\left(r_{j}\right) g_{5}\left(r_{i j}\right) \\
& \left.-2 g_{1}\left(r_{i}\right) g_{3}\left(r_{j}\right) g_{3}\left(r_{i j}\right)-2 g_{3}\left(r_{i}\right) g_{1}\left(r_{j}\right) g_{3}\left(r_{i j}\right)\right]
\end{aligned}
$$

where $\alpha=1.31 a_{0}^{3}$ is the polarizability of $\mathrm{He} ; r_{i}, r_{j}$ are $\mathrm{He}-\mathrm{Li}^{+}$distances; $r_{i j}$ is the He-He distance and $f_{n}(r)$ are damping functions given by:

$$
f_{n}(r)=1-e^{-b r} \sum_{k=0}^{n} \frac{|b r|^{k}}{k !}
$$

with $b$ equal to 2.9 (3.2) for the $\mathrm{He}-\mathrm{Li}^{+}(\mathrm{He}-\mathrm{He})$ interaction. In Eq. (9), $g_{n}\left(r_{i}\right)=$ $f_{n}\left(r_{i}\right) / r_{i}^{n}$. The calculations including the $3 \mathrm{~B}$ term were performed by considering a value of $\beta=9$ (See Eq. (8)) in the ILJ description of the He-He interaction. Quantitative differences in values of the obtained evaporation energies were observed for the smallest sizes $(N \leq 10)$ of $\mathrm{He}_{N} \mathrm{Li}^{+}[189]$. For $\mathrm{Cs}^{+}, 3 \mathrm{~B}$ effects were found of no relevance in He clusters as opposed to the droplets formed by $\mathrm{H}_{2}$ [231].

As the system under consideration gets more complicated with mixture of ions and even molecules, different simplifications may be needed. In the case of light molecules and due to the weak forces involved in the corresponding interactions between helium and the impurity make the pseudoatom approximation a good starting point in which rotation can be neglected [247]. For the smallest sizes rotational contribution and 3B effects can possibly compete as shown for $\left(\mathrm{H}_{2}\right){ }_{N} \mathrm{Cs}^{+}$[231] and related systems [337].

The previous treatments can be also extended when the impurity is a rather large molecule, such as one of the PAHs mentioned in Section 5.2. In those cases the total vdW interaction with the He atoms can be described by means of potential energy surfaces based on an atom-bond pairwise additive scheme with the $\mathrm{C}-\mathrm{C}$ and $\mathrm{C}-\mathrm{H}$ chemical bonds in the PAH considered as interaction centers on the molecular frame [338]. Each atom-bond interaction contributions depends on both the $R_{l}$ distance between the He atom and the bond reference point and the $\theta_{l}$ Jacobi angle which 
describes the orientation of the helium atom relative to the bond axis. The ILJ representation of these pair potentials comprises well depth, $\epsilon_{l}\left(\theta_{l}\right)$, and the location of the atom-bond interaction, $R_{m l}\left(\theta_{l}\right)$, which can be expressed in terms of specific values corresponding to the perpendicular and parallel approaches of the atom to the bond. Estimations of these parameters involved in all these expressions for the case of neutral Cor in He clusters [246] were derived from the polarizability of the interacting partners [339] and fine-tuned through comparison with high level electronic structure predictions. Further applications for Cor molecules embedded in clusters of $\mathrm{H}_{2}$ require to take into account the orientation of the molecular hydrogen relative to the PAH plane [247].

\subsection{Structure and stability}

The PIMC study performed by Nakayama and Yamashita [193] on $\mathrm{He}_{N} \mathrm{Na}^{+}$was probably one of the first theoretical microscopic treatments of a positive alkali ion in nanodroplets of He. In their study, the authors examined the temperature and size dependence of physical quantities such as energy and superfluid fraction. For the largest clusters under consideration, $N>100$, a triple-layer structure of He atoms surrounding the alkali ion was observed. The first shell is rigid-body and solid-like, the second shell displays a superfluid transition between $1 \mathrm{~K}$ and $1.25 \mathrm{~K}$, and the third shell behaves as liquid helium.

The presence of $\mathrm{Li}^{+}, \mathrm{Na}^{+}, \mathrm{K}^{+}$and $\mathrm{Cs}^{+}$in liquid helium were probed by means of variational Monte Carlo (VMC) calculations [194, 195, 340] using the shadow wave function technique. Different properties such as the chemical potential, the local order, the single-particle excitation spectrum, radial density profiles and the effective mass of the ion were obtained. In addition to the actual number of He atoms filling the first shell of atoms, these studies concluded this first shell exhibits an ordered structure forming the so-called snowballs. The microscopic structure of such snowballs depends on the ion. Thus, whereas for $\mathrm{Na}^{+}$and $\mathrm{K}^{+}$the first shell remains solid, for the case of $\mathrm{Li}^{+}$and $\mathrm{Ca}^{+}$, there are exchanges among the He atoms. As a result, dramatic differences in the values of the effective masses (accounting for the actual ion mass and the excess mass due to the snowball around the ion and the back-flow of the surrounding fluid) are found [340]. The helium contribution to the effective translational mass in the case of $\mathrm{He}_{N}$ clusters doped with these alkali ions was also investigated by means of hydrodynamic mass in the investigation by Lehmann [341]. In a series of papers, Gianturco and collaborators also studied the microsolvation of alkali ions [190, 317, 336, 342, 343], providing, for example the number of He atoms filling the first solvation shell of $\mathrm{He}_{N} \mathrm{Li}^{+}$by means of DMC calculations [190]. DMC was also employed in combination with a global optimization through basin-hopping $(\mathrm{BH})$ in the computational investigation of structures and energies of $\mathrm{He}_{N} \mathrm{Na}^{+}$performed by Issaoui et al. [197]. The same method was used in the study of both $\mathrm{He}_{N} \mathrm{~Pb}^{2+}$ and $\mathrm{He}_{N} \mathrm{~Pb}^{+}$clusters performed by Slavíček and Lewerenz [207]. The authors calculated energies and radial density distributions for sizes up to $N=25$ employing ab initio techniques to describe the corresponding analytical many-body potentials including non pairwise additive interactions. Whereas for the case of $\mathrm{Pb}^{2+}$, the first solvation 
shell was found to be closed with $N=12$ He atoms, the effect of spin-orbit coupling affected the structure of the $\mathrm{He}_{N} \mathrm{~Pb}^{+}$clusters which do not develop pronounced solvation shells. Mella and Cargnoni studied the ground state structure and energetics of $\mathrm{He}_{N} \mathrm{Sr}^{+}$and $\mathrm{He}_{N} \mathrm{Ba}^{+}$droplets as possible products of the ${ }^{2} P \leftarrow{ }^{2} S$ excitation process by means of DMC calculations [344].

The path integral ground state Monte Carlo calculations performed by Paolini et al. [48] suggested a distinct behaviour of the surrounding He clusters depending on the ionic impurity. Thus, whereas the alkali - earth $\mathrm{Mg}^{+}$and $\mathrm{Ca}^{+}$produce ${ }^{4} \mathrm{He}$ liquid-like structures ("bubbles"), the lighter $\mathrm{Be}^{+}$and the alkali ions $\mathrm{Li}^{+}$and $\mathrm{Na}^{+}$are embedded by solidlike ("snowball") arrangements. This dependence was further confirmed in the PIMC investigation of Ref. [196], where authors found that the number of He atoms around the ion was determined by the position of the minimum of the corresponding ion-He potential whereas the degree of localization is a function of its depth. Highly ordered solid snowballs were also found for the He clusters surrounding an $\mathrm{Ar}^{+}$ion according to the PIMC calculation performed by Tramonto et al. [173]. In particular, 12 He atoms form an icosahedron in the first shell; 20 He atoms display a dodecahedron structure in the second shell and, finally, 12 more He atoms are located at the vertices of another icosahedron.
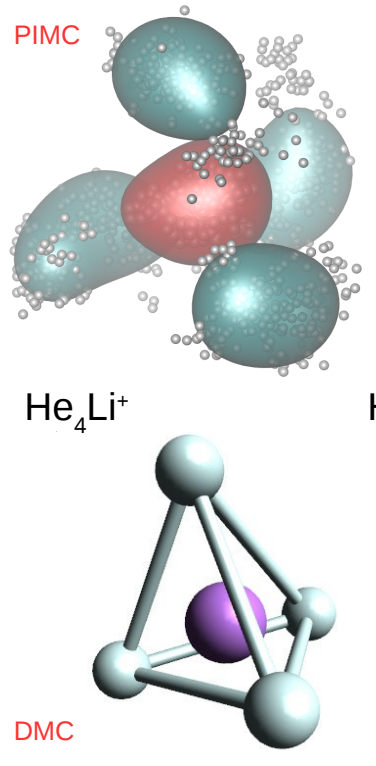

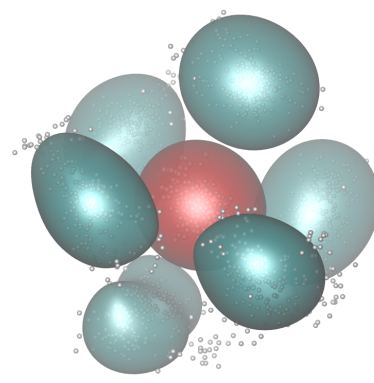

$\mathrm{He}_{6} \mathrm{Li}^{+}$

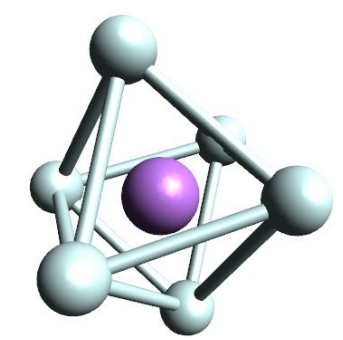

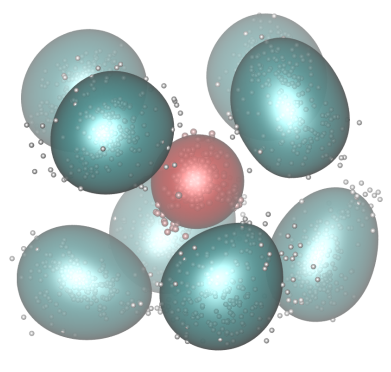

$\mathrm{He}_{8} \mathrm{Li}^{+}$

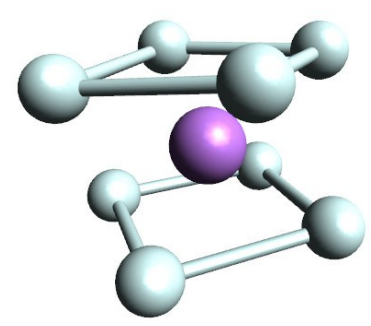

Figure 11. Probability density functions for $\mathrm{He}_{N} \mathrm{Li}^{+}$with $N=4,6$ and 8 obtained with PIMC (top) at $T=2 \mathrm{~K}$ and DMC (bottom) calculations. Figures adapted from Ref. [189].

Methods based on density functional theory (DFT) are also commonly employed as a powerful alternative for the theoretical study of local order around cations inmersed in liquid helium droplets [35]. The apparent structural liquid-solid transformation 


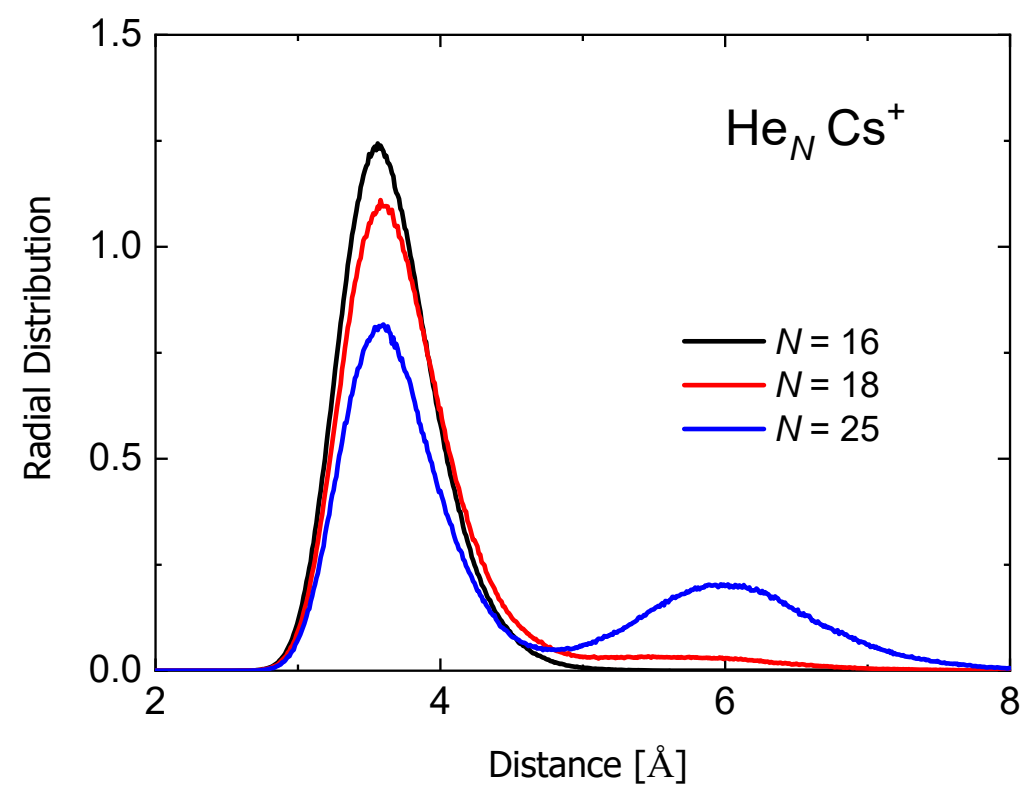

Figure 12. Radial probability densities for $\mathrm{He}_{N} \mathrm{Cs}^{+}$with $N=16,18$ and 25 calculated with a PIMC method at $T=2 \mathrm{~K}$. Results adapted from Ref. [201].

undergone by at least a substantial part of the He droplet by the pressure exerted by the outer electron of a Rydberg atom in scolium-like systems [345, 346] was studied by means of DFT in the case of $\mathrm{Be}^{+}$[347]. Static DFT calculations showed that the equilibrium configuration of helium clusters with either $\mathrm{Rb}^{+}$or $\mathrm{Cs}^{+}$consists of a situation where the impurity is fully solvated inside the droplet [61]. However, time dependent DFT investigations of the dynamics following the photoionization of neutral Rb and Cs initially located in a dimple at the surface of the superfluid ${ }^{4} \mathrm{He}_{N}$ nanodroplet reveals that opposite to the case of $\mathrm{Rb}^{+}$, the $\mathrm{Cs}^{+}$ion is not completely solvated by the He droplet and desorbs from the bulk forming $\mathrm{He}_{N} \mathrm{Cs}^{+}$snowballs. A similar dynamical investigation intiated from a neutral $\mathrm{Ba}$ atom residing in a dimple at the surface of a ${ }^{4} \mathrm{He}_{1000}$ complex showed, that, once ionized, the $\mathrm{Ba}^{+}$cation is solvated with a vortex ring at its equatorial region in its route to the interior of the droplet [348]. Also within the DFT framework, Fiedler et al. [349] calculated hydrodynamical added masses for a series of ions in helium, including the cases of the cations $\mathrm{Be}^{+}, \mathrm{K}^{+}, \mathrm{Ca}^{+}, \mathrm{Sr}^{+}$and $\mathrm{Ba}^{+}$. Ring-polymer molecular dynamics simulations have also been proposed as an useful approach to investigate helium clusters doped by $\mathrm{Na}$ after sudden ionization [350].

More recently, a PIMC method [34] has been applied to investigate the structure and energetics of helium clusters doped with either anions such as $\mathrm{He}^{*-}$ and $\mathrm{He}_{2}^{*-}$ or cations such as $\mathrm{Cs}^{+}[201]$ and $\mathrm{Li}^{+}$[189] solvated in helium. The minimum energy geometries for the clusters $\mathrm{He}_{N} \mathrm{X}$ obtained by means of basin-hopping calculations were employed as the initial configurations for the Monte Carlo simula- 
tion. The analysis in the case of $\mathrm{Li}^{+}$of numerical indicators as the evaporation energy, defined as $\Delta E=E_{N}-E_{N-1}$, or the second difference energies, that is $\Delta_{2} E_{N}=E_{N+1}+E_{N-1}-2 E_{N}$, served to identify particularly stable He shells surrounding the ion for $N=4,6$ and 8 . The agreement observed for $N=6$ and 8 with the experimental findings suggests that those features in the measured mass spectrum correspond to octahedral and parallel square structures, respectively. Probability density functions for these three clusters obtained by means of PIMC at $T=2 \mathrm{~K}$ and DMC calculations are shown in Fig. 11. The study of the solvation of $\mathrm{Cs}^{+}$[201], revealed interesting features for $N=12$ and 17. In particular, on the one hand, for $\mathrm{He}_{12} \mathrm{Cs}^{+}$icosahedral configurations were found to contribute significantly and, on the other hand, indications of the closure of the first solvation layer when 17 He atoms surround the ion impurity were seen. Figure 12 shows the radial probability density for $\mathrm{Cs}^{+}$solvated in clusters of 16, 18 and 25 helium atoms, respectively obtained at $T=2 \mathrm{~K}$ by means of a PIMC calculation. The results show that besides the main peak at $\approx 3.5 \AA$, a second maximum starts to develop at $\approx 6 \AA$ thus indicating that helium atoms added for $N>17$ locate at a second layer once the first shell is full.

Although the examples involving PAHs mentioned at the end of Section 8.1 correspond to the neutral cases, this seems to be a very useful approach to investigate $(\mathrm{PAH})^{+} \mathrm{He}_{N}$ clusters. In fact, after all, due to the large size of the alipathic molecule, the positive charge is largely delocalized as shown in the case of coronene and larger circumcoronene molecule where the partial charges are shown to be very much spread on carbons and hydrogens [351]. Thus, for example, some of the theoretical findings for $\mathrm{He}_{N}$-Cor reported by Rodríguez-Cantano et al. [246] were in remarkably good agreement with experimental results for the $\mathrm{He}_{N}$ Cor $^{+}[215]$.

\section{Conclusions}

Experiments performed at Innsbruck are aimed at probing the properties of positively or negatively charged ions solvated in helium; the size of the solvated species ranges from single atoms to clusters of fullerenes or polycyclic aromatic hydrocarbons. Three approaches have been discussed in detail: First, the ion yield measured as a function of the energy of the incident electrons offers insight into the location of the neutral precursor in the HND, and the events that take place between the primary event (formation of $\mathrm{He}^{+}$or excited $\mathrm{He}^{*}$ ) and ionization of the dopant. Second, the size distributions of $\mathrm{He}_{N} \mathrm{X}^{ \pm}$may reveal particularly stable sizes because the ion abundance is approximately proportional to the dissociation energy. Third, electronic absorption spectra of $\mathrm{He}_{N} \mathrm{X}^{+}$measured by the messenger technique exhibit spectral shifts that keep changing as $N$ is increased. Thus, the messenger technique does not merely signal absorption of a photon, it also measures the interaction of the messenger with the solvated ion and, indirectly, its adsorption site.

The experimental data discussed above provide clues to other properties, including the geometric structure of solvation shells, but without high level theoretical work any conclusion as to the geometric arrangement of helium atoms in $\mathrm{He}_{N} \mathrm{X}^{ \pm}$remains tentative. Experimental data may inspire theoretical studies, and vice versa. Achieving agreement, or at least consistency, between experiment and theory is gratifying 
but discrepancies are no less valuable if they motivate further research. This review includes some of the most recent attempts to provide the theoretical counterpart to the experimental findings. In particular, special emphasis has been made in order to list both the numerical methods commonly used in the study of this kind of clusters and the description of the interactions between the helium atoms and the corresponding impurity. Some examples of the theoretical studies performed by the group in Madrid have been given with some detail.

Unraveling the quantum nature of ${ }^{4} \mathrm{He}$ in $\mathrm{He}_{N} \mathrm{X}^{ \pm}$is another challenge, primarily to theory, because experiments discussed here are more or less blind to them. Molecular beam experiments will readily reveal size effects, but controlling or merely measuring the temperature of the complexes is not yet a viable option. Hopefully, a future review article will report progress in this endeavor.

\section{Acknowledgments}

This work was supported by the Austrian Science Fund FWF (Project Nos. P31149 and P30355), the EU commission, EFRE K-Regio FAENOMENAL (Project No. EFRE 2016-4), the Swedish Research Council (Contract No. 2016-06625), and the MICINN with Grants No. FIS2014-51933-P, FIS2017-83157-P, FIS2014-52172-C2 and FIS2017-83473-C2. 


\section{References}

[1] E. W. Becker, R. Klingelhöfer, and P. Lohse, Z. Naturforsch. A: Astrophys, Phys. Phys. Chem. 161259 (1961).

[2] E. W. Becker, R. Klingelhöfer, and P. Lohse, Z. Naturforsch. A: Astrophys, Phys. Phys. Chem. 15 644-645 (1960). doi: 10.1515/zna-1960-0714.

[3] J. Gspann and G. Krieg, J. Chem. Phys. 61 (10), 4037-4047 (1974) doi: $10.1063 / 1.1681697$.

[4] J. Gspann, Surf. Science 106, 219-224 (1981) doi: 10.1016/0039-6028(81)90204-1.

[5] J. Gspann, Phys. B-C 108 (1-3), 1309-1310 (1981) doi: 10.1016/0378-4363(81)909530 .

[6] J. Gspann and H. Vollmar, J. Low Temp. Phys. 45 (3-4), 343-355 (1981) doi: $10.1007 /$ bf00655138.

[7] J. Gspann, Phys. B 169, 519-520 (1991), doi: 10.1016/0921-4526(91)90305-X.

[8] J. Gspann, Z. Phys. B 98, 405-411 (1995), doi: 10.1007/BF01338415.

[9] S. Stringari and J. Treiner, J. Chem. Phys. 87, 5021-5027 (1987), doi: $10.1063 / 1.452818$.

[10] D.M. Brink and S. Stringari, Z. Phys. D 15, 257-263 (1990), doi: 10.1007/BF01437187.

[11] K. B. Whaley Int. Rev. Phys. Chem. 13 (1) 41-84 (1994) doi: 10.1080/01442359409353290.

[12] E.W. Becker, Z. Phys. D 3, 101-107 (1986), doi: 10.1007/BF01384793.

[13] J. A. Northby, J. Chem. Phys. 115 (22), 10065-10077 (2001) doi: 10.1063/1.1418249.

[14] S. Goyal, D. L. Schutt and G. Scoles, Phys. Rev. Lett. 69 933-936 (1992) doi: 10.1103/PhysRevLett.69.933

[15] S. Goyal, D.L. Schutt and G. Scoles, Acc. Chem. Res. 26, 123-130 (1993), doi: 10.1021/ar00027a008.

[16] A. Scheidemann, J. P. Toennies and J. A. Northby, Phys. Rev. Lett. 64, 1899-1902 (1990), doi: 10.1103/PhysRevLett.64.1899.

[17] R. Fröchtenicht, J. P. Toennies and A. Vilesov, Chem. Phys. Lett. 229, 1-7 (1994), doi: 10.1016/0009-2614(94)01026-9.

[18] M. Hartmann, R. E. Miller, J. P. Toennies and A. Vilesov, Phys. Rev. Lett. 75, 1566-1569 (1995), doi: 10.1103/PhysRevLett.75.1566.

[19] M. Hartmann, F. Mielke, J. P. Toennies, A. F. Vilesov and G. Benedek, Phys. Rev. Lett. 76, 4560-4563 (1996), doi: 10.1103/PhysRevLett.76.4560.

[20] S. Grebenev, J. P. Toennies and A. F. Vilesov, Science 279, 2083-2086 (1998), doi: 10.1126/science.279.5359.2083.

[21] J. P. Toennies and A. F. Vilesov, Angew. Chem. 43 (20), 2622-2648 (2004), doi: 10.1002 /anie.200300611.

[22] M. Barranco, R. Guardiola, S. Hernández, R. Mayol, J. Navarro and M. Pi, J. Low Temp. Phys. 142, 1-81 (2006), doi: 10.1007/s10909-005-9267-0.

[23] J. Tiggesbäumker and F. Stienkemeier, Phys. Chem. Chem. Phys. 9, 4748-4770 (2007), doi: 10.1039/b703575f.

[24] J. P. Toennies, Mol. Phys. 111, 1879-1891 (2013), doi: 10.1080/00268976.2013.802039.

[25] S. F. Yang and A. M. Ellis, Chem. Soc. Rev. 42, 472-484 (2013), doi: $10.1039 / \mathrm{c} 2 \mathrm{cs} 35277 \mathrm{j}$.

[26] A. Mauracher, O. Echt, A. M. Ellis, S. Yang, D. K. Bohme, J. Postler, A. Kaiser, S. Denifl, and P. Scheier, Phys. Rep. 751, 1-90 (2018) doi: 10.1016/j.physrep.2018.05.001.

[27] M. Y. Choi, G. E. Douberly, T. M. Falconer, W. K. Lewis, C. M. Lindsay, J. M. Merritt, P. L. Stiles, and R. E. Miller, Int. Rev. Phys. Chem. 25 (1-2), 15-75 (2006) doi: 10.1063/1.440347.

[28] J. Küpper and J. M. Merritt, Int. Rev. Phys. Chem. 26 (2), 249-287 (2007) doi: $10.1080 / 01442350601087664$.

[29] W. Kong, L. Pei, and J. Zhang, Int. Rev. Phys. Chem. 28 (1), 33-52 (2009) doi: $10.1080 / 01442350802573678$.

[30] M. Mudrich and F. Stienkemeier, Int. Rev. Phys. Chem. 33, 301-339 (2014) doi: 
10.1080/0144235X.2014.937188.

[31] D. Verma, R. M. P. Tanyag, S. M. O. O'Connell and A. F. Vilesov, Adv. Phys.-X 4, 1553569 (2018), doi: 10.1080/23746149.2018.1553569.

[32] M. P. Ziemkiewicz, D. M. Neumark, and O. Gessner, Int. Rev. Phys. Chem. 34 (2), 239-267 (2015) doi: 10.1080/0144235x.2015.1051353.

[33] K. Szalewicz, Int. Rev. Phys. Chem. 27 (2), 273-316 (2008) doi: $10.1080 / 01442350801933485$.

[34] R. Rodríguez-Cantano, T. González-Lezana, and P. Villarreal, Int. Rev. Phys. Chem. 35 (1), 37-68 (2016) doi: 10.1080/0144235X.2015.1132595.

[35] F. Ancilotto, M. Barranco, F. Coppens, J. Eloranta, N. Halberstadt, A. Hernando, D. Mateo, and M. Pi, Int. Rev. Phys. Chem. 36 (4), 621-707 (2017) doi: 10.1080/0144235X.2017.1351672.

[36] P. V. Zandbergen, M. Barranco, F. Cargnoni, M. Drabbels, M. Pi and N. Halberstadt, J. Chem. Phys. 148, 144302 (2018), doi: 10.1063/1.5022863.

[37] B. Thaler, S. Ranftl, P. Heim, S. Cesnik, L. Treiber, R. Meyer, A. W. Hauser, W. E. Ernst and M. Koch, Nat. Commun. 9, 4006 (2018), doi: 10.1038/s41467-018-06413-9.

[38] M. Mudrich, A. C. LaForge, A. Ciavardini, P. O'Keeffe, C. Callegari, M. Coreno, A. Demidovich, M. Devetta, M. Di Fraia, M. Drabbels, P. Finetti, O. Gessner, C. Grazioli, A. Hernando, D. M. Neumark, Y. Ovcharenko, P. Piseri, O. Plekan, K. C. Prince, R. Richter, M. P. Ziemkiewicz, T. Möller, J. Eloranta, M. Pi, M. Barranco and F. Stienkemeier, Nat. Commun. 117 (2020) doi: 10.1038/s41467-019-13681-6.

[39] O. Gessner and A. F. Vilesov, Annu. Rev. Phys. Chem., edited by M. A. Johnson and T. J. Martinez (2019), Vol. 70 pp. 173-198, doi: 10.1146/annurev-physchem-042018052744 .

[40] F. Lackner, A. Schiffmann, M. Lasserus, R. Messner, M. Schnedlitz, H. Fitzek, P. Polt, D. Knez, G. Kothleitner and W. E. Ernst, Eur. Phys. J. D 73, 104 (2019), doi: 10.1140/epjd/e2019-90696-8.

[41] L. Kazak, S. Gode, K. H. Meiwes-Broer and J. Tiggesbäumker, J. Phys. Chem. A 123, 5951-5956 (2019), doi: 10.1021/acs.jpca.9b02880.

[42] J. W. Niman, B. S. Kamerin, L. Kranabetter, D. J. Merthe, J. Suchan, P. Slavicek and V. V. Kresin, Phys. Chem. Chem. Phys. 21, 20764-20769 (2019), doi: 10.1039/c9cp04322e.

[43] K. R. Atkins, Phys. Rev. 116, 1339-1343 (1959) doi: 10.1103/PhysRev.116.1339.

[44] W. W. Johnson and W. I. Glaberson, Phys. Rev. Lett. 29 (4), 214 (1972) doi: 10.1103/PhysRevLett.29.214.

[45] P. J. Knowles and J. N. Murrell, Mol. Phys. 87 (4), 827-833 (1996) doi: doi.org/10.1080/00268979600100551.

[46] W. I. Glaberson and W. W. Johnson, J. Low Temp. Phys. 20, 313-338 (1975) doi: doi.org/10.1007/BF00117800.

[47] M. W. Cole and R. A. Bachman, Phys. Rev. B 15 (3), 1388-1394 (1977) doi: 10.1103/PhysRevB.15.1388.

[48] S. Paolini, F. Ancilotto, and F. Toigo, J. Chem. Phys. 126 (12), 124317 (2007) doi: 10.1063/1.2711813.

[49] A. G. Khrapak and W. F. Schmidt, Low Temp. Phys. 37 (5), 387-391 (2011) doi: doi.org/10.1063/1.3599656.

[50] C. G. Kuper, Phys. Rev. 122, 1007-1011 (1961) doi: 10.1103/PhysRev.122.1007.

[51] M. Rosenblit and J. Jortner, J. Chem. Phys. 124, 194505 (2006) doi: $10.1063 / 1.2192780$.

[52] E. Coccia, F. Marinetti, E. Bodo, and F. A. Gianturco, J. Chem. Phys. 128 (13), 134511 (2008) doi: 10.1063/1.2842082.

[53] E. W. Becker, P. Lohse, and R. Klingelhöfer, Z. Naturforsch. A: Astrophys, Phys. Phys. Chem. A 17 (5), 432-438 (1962) doi: 10.1515/zna-1962-0509.

[54] J. Gspann and H. Vollmar, J. Chem. Phys. 73, 1657-1664 (1980) doi: $10.1063 / 1.440347$.

[55] C. Callegari, K. K. Lehmann, R. Schmied, and G. Scoles, J. Chem. Phys. 115 (22), 10090 (2001) doi: 10.1063/1.1418746 . 
[56] M. Hartmann, N. Portner, B. Sartakov, J. P. Toennies, and A. F. Vilesov, J. Chem. Phys. 110 (11), 5109-5123 (1999) doi: 10.1063/1.479111.

[57] S. F. Yang, S. M. Brereton, M. D. Wheeler, and A. M. Ellis, J. Phys. Chem. A 110 (5), 1791-1797 (2006) doi: 10.1021/jp0544437.

[58] T. Döppner, Th. Diederich, A. Przystawik, N. X. Truong, Th. Fennel, J. Tiggesbäumker and K. H. Meiwes-Broer, Phys. Chem. Chem. Phys. 9, 4639-4652 (2007) doi: 10.1039/B703707D.

[59] S. Müller, M. Mudrich, and F. Stienkemeier, J. Chem. Phys. 131 (4), 044319 (2009) doi: $10.1063 / 1.3180819$.

[60] M. Theisen, F. Lackner, and W. E. Ernst, Phys. Chem. Chem. Phys. 12, 14833 (2010) doi: $10.1039 / \mathrm{c} 0 \mathrm{cp} 01283 \mathrm{a}$.

[61] A. Leal, D. Mateo, A. Hernando, M. Pi, M. Barranco, A. Ponti, F. Cargnoni, and M. Drabbels, Phys. Rev. B 90, 224518 (2014) doi: 10.1103/PhysRevB.90.224518.

[62] U. Henne and J. P. Toennies, J. Chem. Phys. 108 (22), 9327-9338 (1998) doi: $10.1063 / 1.476385$.

[63] M. V. Rama Krishna and K. B. Whaley, Phys. Rev. B 38 (16), 11839-11842 (1988) doi: $10.1063 / 1.476385$.

[64] T. Jiang, C. Kim, and J. A. Northby, Phys. Rev. Lett. 71 (5), 700-703 (1993), doi: 10.1103/PhysRevLett.71.700.

[65] J. P. Toennies and A. F. Vilesov, Ann. Rev. Phys. Chem. 49 (1), 1-41 (1998) doi: 10.1146/annurev.physchem.49.1.1.

[66] C. E. Klots, J. Phys. Chem. 92 (21), 5864-5868 (1988) doi: 10.1021/j100332a005.

[67] J. U. Andersen, E. Bonderup, K. Hansen, P. Hvelplund, B. Liu, U. V. Pedersen, and S. Tomita, Eur. Phys. J. D 24 (1-3), 191-196 (2003) doi: 10.1140/epjd/e2003-00180-y.

[68] M. N. Slipchenko, S. Kuma, T. Momose and A. F. Vilesov, Rev.Sci. Instrum. 73 (10), 3600-3605 (2002) doi: 10.1063/1.1505661.

[69] D. Pentlehner, R. Riechers, B. Dick, A. Slenczka, U. Even, N. Lavie, R. Brown and K. Luria, Rev. Sci. Instrum. 80 (4), 043302 (2009) doi: 10.1063/1.3117196.

[70] D. Verma and A. F. Vilesov, Chem. Phys. Lett. 694 129-134 (2018) doi: 10.1016/j.cplett.2018.01.035.

[71] S. Yang, S. M. Brereton and A. M.Ellis, Rev. Sci. Instrum. 76 (10), 104102 (2005) doi: $10.1063 / 1.2093766$.

[72] C. M. Zhang, Z. G. Zhang, C. S. Huang, Q. Zhang and Y. Chen, Chin. J. Chem. Phys., 26 270-276 (2013) doi: 10.1063/1674-0068/26/03/270-276.

[73] L. Lei, Y. Z. Yao, J. Zhang, D. Tronrud, W. Kong, C. Z. Zhang, L. Xue, L. Donto and M. Rapacioli, J. Phys. Chem. Lett., 11 724-729 (2020) doi: 10.1021/acs.jpclett.9b03603.

[74] Y. T. He, J. Zhang, Y. Li, W. M. Freund and W. Kong, Rev. Sci. Instrum., 868 (2015) doi: 10.1063/1.4928107.

[75] F. Bierau, P. Kupser, G. Meijer and G. von Helden, Phys. Rev. Lett. 105133402 (2010) doi: 10.1103/PhysRevLett.105.13340.

[76] H. Buchenau, E. L. Knuth, J. Northby, J. P. Toennies and C. Winkler, J. Chem. Phys. 92 6875-6889 (1990) doi: 10.1063/1.458275.

[77] D. J. Merthe and V. V. Kresin, J. Phys. Chem. Lett. 7 4879-4883 (2016). doi: $10.1063 / 1.458275$.

[78] L. F. Gómez, E. Loginov, R. Sliter and A. F. Vilesov, J. Chem. Phys. 135154201 (2011) doi: 10.1063/1.3650235.

[79] F. Stienkemeier, J. Higgins, C. Callegari, S. I. Kanorsky, W. E. Ernst, G. Scoles, Z. Phys. D 38 253-263 (1996) doi: 10.1007/s004600050090.

[80] H. Schöbel, P. Bartl, C. Leidlmair, S. Denifl, O. Echt, T. D. Märk and Scheier, Eur. Phys. J. D 63 (2), 209-214, (2011) doi: 10.1140/epjd/e2011-10619-1.

[81] M. Alghamdi, J. Zhang, A. Oswalt, J. J. Porter, R. A. Mehl and W. Kong, J. Phys. Chem. A, 121 6671-6678 (2017) doi: 10.1021/acs.jpca.7b05718.

[82] R. Sliter, L. F. Gomez, J. Kwok and A. Vilesov, Chem. Phys. Lett. 600 29-33 (2014) doi: $10.1016 /$ j.cplett.2014.03.053

[83] R. M. P. Tanyag, C. F. Jones, C. Bernando, S. M. O. O'Connell, D. Verma and A. F. 
Vilesov, in Experiments with Large Superfluid Helium Nanodroplets, Cold Chemistry: Molecular Scattering and Reactivity Near Absolute Zero, edited by The Royal Society of Chemistry (2018) Chap. 8 pp. 389-443.

[84] P. Thaler, A. Volk, F. Lackner, J. Steurer, D. Knez, W. Grogger, F. Hofer and W. E. Ernst, Phys. Rev. B 90155442 (2014) doi: 10.1103/PhysRevB.90.155442

[85] H. Schöbel, P. Bartl, C. Leidlmair, M. Daxner, S. Zöttl, S. Denifl, T. D. Märk, P. Scheier, D. Spångberg, A. Mauracher and D. K. Bohme, Phys. Rev. Lett. 105243402 (2010) 10.1103/PhysRevLett.105.243402

[86] R. M. Cleaver and C. M. Lindsay, Cryogenics 52 389-397 (2012) doi: 10.1016/j.cryogenics.2012.03.004.

[87] M. Lewerenz, B. Schilling, J. P. Toennies, Chem. Phys. Lett. 206 381-387 (1993) doi: 10.1016/0009-2614(93)85569-A.

[88] V. Ghazarian, J. Eloranta and V. A. Apkarian, Rev. Sci. Instrum. 73 3606-3613 (2002) doi: 10.1063/1.1505662

[89] K. Allweins, L. M. Qiu and G. Thummes in Advances in Cryogenic Engineering: J.G. Weisend, J. Barclay, S. Breon, J. Demko, M. DiPirro, J.P. Kelley, P. Kittel, A. Klebaner, J. Lock, T. Peterson, J. Pfotenhauer, A. Rowe, S. VanSciver, M. Zagarola, A. Zeller (Eds.), Vols. 53a and 53b, (Amer. Inst. Physics, Melville, 2008), pp. 109-116.

[90] G. Dubuis, X. He and I. Božovic, Rev. Sci. Instrum. 854 (2014) doi: $10.1063 / 1.4896049$

[91] A. C. LaForge, M. Drabbels, N. B. Brauer, M. Coreno, M. Devetta, M. Di Fraia, P. Finetti, C. Grazioli, R. Katzy, V. Lyamayev, T. Mazza, M. Mudrich, P. O'Keeffe, Y. Ovcharenko, P. Piseri, O. Plekan, K. C. Prince, R. Richter, S. Stranges, C. Callegari, T. Möller, F. Stienkemeier, Sci. Rep., 43621 (2014) doi: 10.1038/srep03621

[92] D. Buchta, S. R. Krishnan, N. B. Brauer, M. Drabbels, P. O'Keeffe, M. Devetta, M. Di Fraia, C. Callegari, R. Richter, M. Coreno, K. C. Prince, F. Stienkemeier, J. Ullrich, R. Moshammer, M. Mudrich J. Chem. Phys. 139084301 (2013) doi: 10.1021/jp401424w.

[93] K. von Haeften, T. Laarmann, H. Wabnitz and T. Möller, J. Phys. B 38 S373-S386 (2005) doi: 10.1088/0953-4075/38/2/028.

[94] R. Fröchtenicht, U. Henne, J. P. Toennies, A. Ding, M. Fieber-Erdmann and T. Drewello, J. Chem. Phys. 104 2548-2556 (1996) doi: 10.1063/1.471009.

[95] D. A. Thomas, E. Mucha, S. Gewinner, W. Schöllkopf, G. Meijer and G. von Helden J. Phys. Chem. Lett., 9 2305-2310 (2018) doi: 10.1021/acs.jpclett.8b00664.

[96] H. Odaka and M. Ichihashi, Eur. Phys. J. D 716 (2017) doi: 10.1140/epjd/e201780015-0

[97] J. Zhang, L. Chen, W. M. Freund and W. Kong, J. Chem. Phys. 1437 (2015). doi: $10.1063 / 1.4928689$

[98] P. Claas, S.-O. Mende and F. Stienkemeier, Rev. Sci. Instrum. 744071 (2003). doi: $10.1063 / 1.1602943$

[99] S. Denifl, F. Zappa, I. Mähr, J. Lecointre, M. Probst, T. D Märk and P. Scheier, Phys. Rev. Lett. 97043201 (2006) doi: 10.1103/PhysRevLett.97.043201

[100] A. Mauracher, M. Daxner, J. Postler, S. E. Huber, S. Denifl, P. Scheier and J. P. Toennies, J. Phys. Chem. Lett. 5 2444-2449 (2014) doi: 10.1021/jz500917z

[101] B. E. Callicoatt, K. Förde, L. F. Jung, T. Ruchti and K. C. Janda J. Chem. Phys. 109 10195-10200 (1998) doi: 10.1063/1.477713

[102] K. R. Atkins in Course XXI on Liquid Helium, Proceedings of International School of Physics Enrico Fermi: G. Careri (Ed.) (Academic, New York, 1963), pp. 403-413

[103] H. Buchenau, J. P. Toennies and J. A. Northby, J. Chem. Phys. 95 8134-8148 (1991) doi: $10.1063 / 1.461294$

[104] N. Halberstadt and K. C. Janda Chem. Phys. Lett. 282 409-412 (1998) doi: 10.1016/S0009-2614(97)01276-1

[105] A. M. Ellis and S. F. Yang, Phys. Rev. A, 76032714 (2007) doi: 10.1103/PhysRevA.76.032714.

[106] W. K. Lewis, C. M. Lindsay, R. J. Bemish and R. E. Miller, J. Am. Chem. Soc. 127 7235-7242 (2005) doi: 10.1021/ja042489s.

[107] L. Tiefenthaler, J. Ameixa, P. Martini, S. Albertini, L. Ballauf, M. Zankl, M. Goulart, 
F. Laimer, K. von Haeften, F. Zappa and P. Scheier, Rev. Sci. Instrum. 9111 (2020) doi: $10.1063 / 1.5133112$.

[108] B. E. Callicoatt, K. Förde, T. Ruchti, L. L. Jung, K. C. Janda and N. Halberstadt J. Chem. Phys. 108 9371-9382 (1998) doi: 10.1063/1.476389

[109] G. Lakits, F. Aumayr, M. Heim and H. Winter, Phys. Rev. A 42 5780(R) (1990) doi: 10.1103/PhysRevA.42.5780.

[110] H. D. Hagstrum, Phys. Rev. 96 (2) 325 (1954) doi: 10.1103/PhysRev.96.325.

[111] K. Töglhofer, F. Aumayr, H. Kurz, H. P. Winter, P. Scheier and T. D. Märk, J. Chem. Phys. 99 (10) 8254-8261 (1993) doi: 10.1063/1.465651.

[112] F. Laimer, L. Kranabetter, L. Tiefenthaler, S. Albertini, F. Zappa, A. M. Ellis, M. Gatchell and P. Scheier, Phys. Rev. Lett. 123165301 (2019) doi: 10.1103/PhysRevLett.123.165301.

[113] A. Günther, P. Nieto, D. Müller, A. Sheldrick, D. Gerlich and O. Dopfer, J. Mol. Spectrosc., 332 8-15 (2017) doi: 10.1016/j.jms.2016.08.017.

[114] E. K. Campbell, M. Holz, D. Gerlich, J. P. Maier Nature, 523322 (2015) doi: 10.1038/nature14566.

[115] J. Roithová, A. Gray, E. Andris, J. Jašík and D. Gerlich, Acc. Chem. Res. 49 223-230 (2016) doi: 10.1021/acs.accounts.5b00489.

[116] D. Gerlich, J. Jašík and J. Roithová, Int. J. Mass Spectrom. 438 78-86 (2019) doi: 10.1016/j.ijms.2018.12.018

[117] N. Kobayashi, T. Kojima and Y. Kaneko, J. Phys. Soc. Jpn. 57 1528-1531 (1988) doi: 10.1143/JPSJ.57.1528

[118] N. Kobayashi, T. Kojima and Y. Kaneko, Z. Phys. D-Atoms Mol. Clusters, 23 181185 (1992) doi: 10.1007/BF01436742.

[119] N. Kobayashi, T. Kojima and Y. Kaneko, Z. Phys. D-Atoms Mol. Clusters 22 (3) 645-650 (1992) doi: 10.1007/BF01426367.

[120] H. Tanuma, J. Sanderson and N. Kobayashi, J. Phys. Soc. Jpn. 68 2570-2575 (1999) doi: 10.1143/JPSJ.68.2570.

[121] T. Ruchti, B. E. Callicoatt and K. C. Janda, Phys. Chem. Chem. Phys. 2 4075-4080 (2000) doi: 10.1039/B002051F.

[122] L. An der Lan, P. Bartl, C. Leidlmair, R. Jochum, S. Denifl, O. Echt and P. Scheier Chem. Eur. J. 18 (14) 4411-4418 (2012) 10.1002/chem.201103432.

[123] M. Kuhn, M. Renzler, J. Postler, S. Ralser, S. Spieler, M. Simpson, H. Linnartz, A. G. G. M. Tielens, J. Cami, A. Mauracher, Y. Wang, M. Alcamí, et al., Nature Comm. 7, 13550 (2016) doi: 10.1038/.

[124] C. Leidlmair, Y. Wang, P. Bartl, H. Schöbel, S. Denifl, M. Probst, M. Alcamí, F. Martín, H. Zettergren, K. Hansen, O. Echt, and P. Scheier, Phys. Rev. Lett. 108, 076101 (2012) doi: 10.1103/PhysRevLett.108.076101.

[125] M. Harnisch, N. Weinberger, S. Denifl, P. Scheier and O. Echt Mol. Phys. 113 21912196 (2015) doi: 10.1080/00268976.2015.1018357

[126] M. Goulart, M. Gatchell, L. Kranabetter, M. Kuhn, P. Martini, N. Gitzl, M. Rainer, J. Postler, P. Scheier and A. M. Ellis Phys. Chem. Chem. Phys. 20 9554-9560 (2018) doi: 10.1039/C8CP01273C.

[127] F. Ferreira da Silva, P. Waldburger, S. Jaksch, A. Mauracher, S. Denifl, O. Echt, T. D. Märk and P. Scheier Chem.-Eur. J., 15 7101-7108 (2009) doi: 10.1002/chem.200802554

[128] A. Bartelt, J. D. Close, F. Federmann, N. Quaas, and J. P. Toennies, Phys. Rev. Lett. 77 (17), 3525 (1996) doi: 10.1103/PhysRevLett.77.3525.

[129] A. Nakayama and K. Yamashita, J. Chem. Phys. 114 (2), 780 (2001) doi: $10.1063 / 1.1322071$.

[130] F. Stienkemeier, F. Meier, and H. O. Lutz, J. Chem. Phys. 107 (24), 10816 (1997) doi: $10.1063 / 1.474198$.

[131] F. Stienkemeier, F. Meier, and H. O. Lutz, Eur. Phys. J. D 9 (1), 313 (1999) doi: 10.1007/PL00010927.

[132] J. Reho, U. Merker, M. R. Radcliff, K. K. Lehmann, and G. Scoles, J. Chem. Phys. 112 (19), 8409 (2000) doi: 10.1063/1.481444. 
[133] J. Navarro, D. Mateo, M. Barranco, and A. Sarsa, J. Chem. Phys. 136 (5), 054301 (2012) doi: 10.1063/1.3675919.

[134] R. Guardiola, J. Navarro, D. Mateo, and M. Barranco, J. Chem. Phys. 131 (17), 174110 (2009) doi: 10.1063/1.3258273.

[135] O. Bünermann, M. Dvorak, F. Stienkemeier, A. Hernando, R. Mayol, M. Pi, M. Barranco, and F. Ancilotto, Phys. Rev. B 79 (21), 214511 (2009) doi: 10.1103/PhysRevB.79.214511.

[136] A. Hernando, R. Mayol, M. Pi, M. Barranco, F. Ancilotto, O. Bünermann, and F. Stienkemeier, J. Phys. Chem. A 111 (31), 7303 (2007) doi: 10.1021/jp0701385.

[137] D. López-Durán, R. Rodríguez-Cantano, T. González-Lezana, G. Delgado-Barrio, P. Villarreal, and F. A. Gianturco, Eur. Phy. J. D 66 (7), 1 (2012) doi: 10.1140/epjd/e2012-30202-6.

[138] R. Rodríguez-Cantano, T. González-Lezana, P. Villarreal, D. López-Durán, F. A. Gianturco, and G. Delgado-Barrio, Int. J Quant. Chem. 114 (19), 1318-1326 (2014) doi: $10.1002 /$ qua. 24622 .

[139] M. Theisen, F. Lackner, and W. E. Ernst, J. Chem. Phys. 135, 074306 (2011) doi: $10.1063 / 1.3624840$.

[140] J. von Vangerow, F. Coppens, A. Leal, M. Pi, M. Barranco, N. Halberstadt, F. Stienkemeier and M. Mudrich, J. Phys. Chem. Lett. 8 (1) 307-312 (2017) doi: 10.1021/acs.jpclett.6b02598.

[141] A. Sieg, J. von Vangerow, F. Stienkemeier, O. Dulieu, and M. Mudrich, J. Phys. Chem. A 120 (39), 7641-7649 (2016) doi: 10.1021/acs.jpca.6b05732.

[142] J. Jortner, Z. Phys. D 24 (3), 247-275 (1992) doi: 10.1007/BF0142574 .

[143] E. Cheng, M. W. Cole, J. Dupontroc, W. F. Saam, and J. Treiner, Rev. Mod. Phys. 65 (2), 557-567 (1993) doi: 10.1103/RevModPhys.65.557.

[144] F. Ancilotto, M. Barranco, E. S. Hernandez, A. Hernando, and M. Pi, Physical Review B 79 (10), 104514 (2009) doi: 10.1103/PhysRevB.79.104514.

[145] F. Federmann, K. Hoffmann, N. Quaas, and J. P. Toennies, Eur. Phys. J. D 9 (1-4), 11-14 (1999) doi: 10.1007/pl00010922.

[146] T. Diederich, J. Tiggesbäumker, and K. H. Meiwes-Broer, Journal of Chem. Phys. 116 (8), 3263-3269 (2002) doi: 10.1063/1.1424310.

[147] E. Loginov, L. F. Gomez, N. Chiang, A. Halder, N. Guggemos, V. V. Kresin, and A. F. Vilesov, Phys. Rev. Lett. 106 (23), 233401 (2011) doi: 10.1103/PhysRevLett.106.233401.

[148] A. A. Scheidemann, V. V. Kresin, and H. Hess, J. Chem. Phys. 107 (8), 2839-2844 (1997) doi: 10.1063/1.474642.

[149] S. Vongehr, A. A. Scheidemann, C. Wittig, and V. V. Kresin, Chem. Phys. Lett. 353 (1-2), 89-94 (2002) doi: 10.1016/S0009-2614(02)00025-8.

[150] Y. Ren and V. V. Kresin, Phys. Rev. A 76 (4), 043204 (2007) doi: 10.1103/PhysRevA.76.043204.

[151] L. An der Lan, P. Bartl, C. Leidlmair, H. Schöbel, S. Denifl, T. D. Märk, A. M. Ellis, and P. Scheier, Phys. Rev. B 85 (11), 115414 (2012) doi: 10.1103/PhysRevB.85.115414.

[152] L. An der Lan, P. Bartl, C. Leidlmair, H. Schöbel, R. Jochum, S. Denifl, T. D. Märk, A. M. Ellis, and P. Scheier, J. Chem. Phys. 135 (4), 044309 (2011) doi: $10.1063 / 1.3610388$.

[153] C. Stark and V. V. Kresin, Phys. Rev. B 81, 085401 (2010) doi: 10.1103/PhysRevB.81.085401.

[154] F. Calvo, Phys. Rev. B 95 (3), 035429 (2017) doi: 10.1103/PhysRevB.95.035429.

[155] J. Höller, E. Krotscheck, and R. E. Zillich, Eur. Phys. J. D 69 (8), 198 (2015) doi: 10.1140/epjd/e2015-60280-7.

[156] M. Brack, Rev. Mod. Phys. 65 (3), 677-732 (1993) doi: 10.1103/RevModPhys.65.677.

[157] W. A. De Heer, Rev. Mod. Phys. 65 (3), 611-676 (1993) doi: 10.1103/RevModPhys.65.611.

[158] M. Shcherbinin, A. C. LaForge, M. Hanif, R. Richter and M. Mudrich, J. Phys. Chem. A 122 (7) 1855-1860 (2018) doi: 10.1021/acs.jpca.7b12506. 
[159] L. B. Ltaief, M. Shcherbinin, S. Mandal, S. Krishnan, R. Richter, T. Pfeifer and M. Mudrich (2020) www.arXiv:2006.08032

[160] M. Renzler, M. Daxner, L. Kranabetter, A. Kaiser, A. W. Hauser, W. E. Ernst, A. Lindinger, R. Zillich, P. Scheier, and A. M. Ellis, J. Chem. Phys. 145, 181101 (2016) doi: 10.1063/1.4967405.

[161] J. E. Campana, T. M. Barlak, R J. Colton, J. J. Decorpo, J. R. Wyatt and B. I. Dunlap, Phys. Rev. Lett. 47 (15) 1046-1049 (1981) doi: 10.1103/PhysRevLett.47.1046.

[162] O. Echt, K. Sattler and E. Recknagel, Phys. Rev. Lett. 47 (16): 1121-1124 (1981) doi: 10.1103/PhysRevLett.47.1121.

[163] W. D. Knight, K. Clemenger, W. A. de Heer, W. A. Saunders, M. Y. Chou and M. L. Cohen Phys. Rev. Lett. 52 (24) 2141-2143 (1984) doi: 10.1103/PhysRevLett.52.2141.

[164] H. W. Kroto, J. R. Heath, S. C. O'Brien, R. F. Curl and R. E. Smalley Nature 318 162-163 (1985) doi: 10.1038/318162a0.

[165] T. P. Martin Phys. Rep. 273 (4) 199-241 (1996) doi: 10.1016/0370-1573(95)00083-6.

[166] R. F. Curl and R. E. Smalley Science 242 (4881) 1017-1022 (1988) doi: 10.1126/science.242.4881.1017.

[167] K. Hansen and U. Näher Phys. Rev. A 60 (2) 1240-1250 (1999) doi: 10.1103/PhysRevA.60.1240.

[168] S. Prasalovich, K. Hansen, M. Kjellberg, V. N. Popok and E. E. B. Campbell J. Chem. Phys. 123084317 (2005) doi: 10.1063/1.2008948.

[169] W. A. de Heer, W. D. Knight, M. Y. Chou and M. L. Cohen Solid State Phys.-Adv. Res. App. 40 93-181 (1987) doi: 10.1016/S0081-1947(08)60691-8.

[170] M. Brack, O. Genzken and K. Hansen Z. Phys. D 19 (1-4): 51-53 (1991) doi: 10.1007/BF01448253.

[171] M. Brack, O. Genzken and K. Hansen Z. Phys. D 21 (1) 65-81 (1991) doi: 10.1007/BF01448253.

[172] P. Bartl, C. Leidlmair, S. Denifl, P. Scheier and O. Echt J. Phys. Chem. A 118 (37) 8050-8059 (2014) doi: 10.1021/jp406540p.

[173] F. Tramonto, P. Salvestrini, M. Nava and D. E. Galli J. Low Temp. Phys. 180 29-36 (2015) doi: 10.1007/s10909-014-1266-6.

[174] Hansen, K. in Statistical Physics of Nanoparticles in the Gas Phase, Springer Series on Atomic, Optical, and Plasma Physics 73 (2nd Ed. Heidelberg, 2019).

[175] C. E. Klots Nature 327 222-223 (1987) doi: 10.1038/327222a0.

[176] F. Calvo, J. Phys. Chem. A 119 (23) 5959-5970 (2015) doi: 10.1021/jp510799h.

[177] F. Grandinetti Int. J. Mass Spect. $237 \quad 243-267 \quad$ (2004) doi: 10.1016/j.ijms.2004.07.012.

[178] F. Grandinetti Eur. J. Mass Spect. 17 (5) 423-463 (2011) doi: 10.1255/ejms.1151.

[179] F. Marinetti and F. A. Gianturco Chem. Phys. 399 205-212 (2012) doi: 10.1016/j.chemphys.2011.07.003.

[180] P. Bartl, S. Denifl, P. Scheier and O. Echt Phys. Chem. Chem. Phys. 15 16599-16604 (2013) doi: 10.1039/C3CP52550C.

[181] M. Mahmoodi-Darian, P. Martini, L. Tiefenthaler, J. Kocisek, P. Scheier and O. Echt J. Phys. Chem. A 123 (48) 10426-10436 (2019) doi: 10.1021/acs.jpca.9b09496

[182] K. Gluch, S. Matt-Leubner, O. Echt, R. Deng, J. U. Andersen, P. Scheier and T. D. Märk Chem. Phys. Lett. 385 449-455 (2004) doi: 10.1016/j.cplett.2003.12.113.

[183] B. Concina, K. Gluch, S. Matt-Leubner, O. Echt, P. Scheier and T. D. Märk Chem. Phys. Lett. 407 (4-6) 464-470 (2005) doi: 10.1016/j.cplett.2005.03.13.

[184] J. Borggreen, K. Hansen, F. Chandezon, T. Døssing, M. Elhajal and O. Echt Phys. Rev. A 62 (1) 013202 (2000) doi: 10.1103/PhysRevA.62.013202.

[185] F. Mezzacapo and M. Boninsegni Phys. Rev. Lett. 100145301 (2008) doi: 10.1103/PhysRevLett.100.145301.

[186] L. Lundberg, P. Bartl, C. Leidlmair, P. Scheier and M. Gatchell Molecules 25 (5) 1066 (2020) doi: 10.3390/molecules25051066.

[187] F. A. Gianturco and F. Filippone Comp. Phys. Comm. 145 (1) 78-96 (2002) doi: 10.1016/s0010-4655(02)00148-0.

[188] P. Bartl, C. Leidlmair, S. Denifl, P. Scheier and O. Echt ChemPhysChem 14 (1) 
227-232 (2013) doi: 10.1002/cphc.201200664.

[189] M. Rastogi, C. Leidlmair, L. An der Lan, J. Ortiz de Zárate, R. Pérez de Tudela, M. Bartolomei, M. I. Hernández, J. Campos-Martínez, T. González-Lezana, J. Hernández-Rojas, J. Bretón, P. Scheier, et al., Phys. Chem. Chem. Phys. 20, 2556925576 (2018) doi: 10.1039/C8CP04522D.

[190] C. Di Paola, F. Sebastianelli, E. Bodo, I. Baccarelli, and F. A. Gianturco, J. Chem. Theor. Comp. 1, 1045-1054 (2005) doi: 10.1021/ct050072m.

[191] E. Coccia, E. Bodo, F. Marinetti, F. A. Gianturco, E. Yildrim, M. Yurtsever and E. Yurtsever J. Chem. Phys. 126124319 (2007) doi: 10.1063/1.2712437.

[192] F. Marinetti, L. I. Uranga-Pina, E. Coccia, D. López-Duran, E. Bodo and F. A. Gianturco J. Phys. Chem. A 111 12289-12294 (2007) doi: 10.1021/jp0748361.

[193] A. Nakayama and K. Yamashita, J. Chem. Phys. 112 (24), 10966-10975 (2000) doi: $10.1063 / 1.481736$.

[194] D. E. Galli, M. Buzzacchi, and L. Reatto, J. Chem. Phys. 115 (22), 10239 (2001) doi: $10.1063 / 1.1414317$.

[195] M. Rossi, M. Verona, D. E. Galli, and L. Reatto, Phys. Rev. B 69, 212510 (2004) doi: 10.1103/PhysRevB.69.212510.

[196] D. E. Galli, D. M. Ceperley, and L. Reatto, J. Phys. Chem. A 115 (25), 7300-7309 (2011) doi: 10.102/jp200617a.

[197] N. Issaoui, K. Abdessalem, H. Ghalla, S. J. Yaghmour, F. Calvo, and B. Oujia, J. Chem. Phys. 141 (17), 174316 (2014) doi: 10.1063/1.4900873. 6.

[198] F. Calvo, in Sodium Dopants in Helium Clusters: Structure, Equilibrium and Submersion Kinetics Eds. T. E. Simos, Z. Kalogiratou and T. Monovasilis (Int. Conf. Comp. Methods Sci Eng., AIP, 2015) 1702 pp. 050003 doi: 10.1063/1.4938785.

[199] M. Laajimi, S. Mtiri and H. Ghalla Theo. Chem. Acc. 139 (2) 40 (2020) doi: 10.1007/s00214-020-2556-5.

[200] L. Kranabetter, M. Goulart, A. Aleem, T. Kurzthaler, M. Kuhn, E. Barwa, M. Renzler, P. Scheier and O. Echt, J. Phys. Chem. C 121 10887-10892 (2017) doi: 10.1021/acs.jpcc.6b12057.

[201] R. Pérez de Tudela, P. Martini, M. Goulart, P. Scheier, F. Pirani, J. HernándezRojas, J. Bretón, J. Ortiz de Zárate, M. Bartolomei, T. González-Lezana, M. I. Hernández, J. Campos-Martínez, et al., J. Chem. Phys. 150 (15), 154304 (2019) doi: 10.1063/1.5092566.

[202] S. Raggl, N. Gitzl, P. Martini, P. Scheier and O. Echt, Eur. Phys. J. D 72130 (2018) doi: 10.1140/epjd/e2018-90150-7.

[203] T. Döppner, T. Diederich, S. Gode, A. Przystawik, J. Tiggesbäumker and K. H. Meiwes-Broer J. Chem. Phys. 126 (24) 244513 (2007) doi: 10.1063/1.2745294.

[204] P. Martini, L. Kranabetter, M. Goulart, B. Rasul, M. Gatchell, P. Scheier and O. Echt J. Phys. Chem. A 123 9505-9513 (2019) doi: 10.1021/acs.jpca.9b06715.

[205] X. P. Bu and C. L. Zhong Chem. Phys. Lett. 392 (1-3) 181-186 (2004) doi: 10.1016/j.cplett.2004.05.079.

[206] X. P. Bu and C. L. Zhong J. Mol. Struct.-Theochem 726 (1-3) 99-105 (2005) doi: 10.1016/j.theochem.2005.04.008.

[207] P. Slavíček and M. Lewerenz, Phys. Chem. Chem. Phys. 12, 1152-1161 (2010) doi: 10.1039/B918186E.

[208] E. Coccia, F. Marinetti, E. Bodo and F. A. Gianturco ChemPhysChem 9 1323-1330 (2008) doi: 10.1002/cphc.200800132.

[209] J. N. Murrell, F. Y. Naumkin and C. R. Griffiths Mol. Phys. 99 (2) 115-132 (2001) doi: 10.1080/00268970010008342.

[210] F. Ferreira da Silva, P. Bartl, S. Denifl, O. Echt, T. D. Märk and P. Scheier Phys. Chem. Chem. Phys. 11 (42) 9791-9797 (2009) doi: 10.1039/b913175b.

[211] M. Lewerenz, B. Schilling and J. P. Toennies J. Chem. Phys. 102 (20) 8191-8206 doi: 10.1063/1.469231.

[212] J. H. Kim, D. S. Peterka, C. C. Wang and D. M. Neumark J. Chem. Phys. 124 (21) 214301 (2006) doi: 10.1063/1.2202313.

[213] H. Tanuma, H. Hidaka and N. Kobayashi AIP Conf. Proc. Physics of Electronic and 
Atomic Collisions 500 699-703 (2000) doi: 10.1063/1.1302699.

[214] M. Gatchell, P. Martini, F. Laimer, M. Goulart, F. Calvo, and P. Scheier, Faraday Discuss. 217, 276-289 (2019) doi: 10.1039/C8FD00178B.

[215] T. Kurzthaler, B. Rasul, M. Kuhn, A. Lindinger, P. Scheier and A. M. Ellis J. Chem. Phys. 145 (6) 064305 (2016) doi: 10.1063/1.4960611.

[216] F. Calvo Comp. Theo. Chem. 1107 2-6 (2017) doi: 10.1016/j.comptc.2016.09.027.

[217] M. Goulart, M. Kuhn, B. Rasul, J. Postler, M. Gatchell, H. Zettergren, P. Scheier and O. Echt Phys. Chem. Chem. Phys. 19 27968-27973 (2017) doi: 10.1039/C7CP04999D.

[218] F. Calvo, Phys. Rev. B 85 (6) 060502(R) (2012) doi: 10.1103/PhysRevB.85.060502.

[219] S. Jaksch, A. Mauracher, A. Bacher, S. Denifl, F. Ferreira da Silva, H. Schöbel, O. Echt, T. D. Märk, M. Probst, D. K. Bohme and P. Scheier J. Chem. Phys. 129 (22) 224306 (2008) doi: 10.1063/1.3035833.

[220] C. Leidlmair, P. Bartl, H. Schöbel, S. Denifl, M. Probst, P. Scheier and O. Echt Astrophys. J. Lett. 738 L4 (2011) doi: 10.1088/2041-8205/738/1/L4.

[221] A. Kaiser, C. Leidlmair, P. Bartl, S. Zöttl, S. Denifl, A. Mauracher, M. Probst, P. Scheier and O. Echt J. Chem. Phys. 138 (7) 074311 (2013) doi: 10.1063/1.4790403.

[222] K. Hiraoka and T. Mori, J. Chem. Phys. 90 (12), 7143-7149 (1989) doi: $10.1063 / 1.456245$.

[223] M. Renzler, M. Kuhn, A. Mauracher, A. Lindinger, P. Scheier and A. M. Ellis Phys. Rev. Lett. 117273001 (2016) doi: 10.1103/PhysRevLett.117.273001.

[224] F. Calvo and E. Yurtsever J. Chem. Phys. 148 (10) 102305 (2018) doi: 10.1063/1.4990612.

[225] B. H. Kohno, J. D. Mallory and V. A. Mandelshtam J. Chem. Phys. 150 (20) 204305 (2019) doi: 10.1063/1.5099255.

[226] R. Clampitt and D. K. Jefferies Nature 226 (5241) 141-142 (1970) doi: $10.1038 / 226141 \mathrm{a} 0$.

[227] M. Barbatti,G. Jalbert and M. A. C. Nascimento J. Chem. Phys. 114 (5) 2213-2218 (2001) doi: 10.1063/1.1338978.

[228] K. R. S. Chandrakumar and S. K. Ghosh Chem. Phys. Lett. 447 (4-6) 208-214 (2007).

[229] A. Ponzi, F. Marinetti and F. A. Gianturco Phys. Chem. Chem. Phys. 11 (20) 38683874 (2009) doi: 10.1039/b820190k.

[230] M. Barbatti, G. Jalbert and M. A. C. Nascimento J. Phys. Chem. 106 (3) 551-555 (2002) doi: 10.1021/jp013159m

[231] J. Ortiz de Zárate, M. Bartolomei, T. González-Lezana, J. Campos-Martínez, M. I. Hernández, R. Pérez de Tudela, J. Hernández-Rojas, J. Bretón, F. Pirani, L. Kranabetter, P. Martini, M. Kuhn, et al., Phys. Chem. Chem. Phys. 21, 15662-15668 (2019) doi: 10.1039/C9CP02017A.

[232] P. R. Kemper, P. Weis, M. T. Bowers and P. Maitre J. Amer. Chem. Soc. 120 (51) 13494-13502 (1998) doi: 10.1021/ja9822658.

[233] M. J. Manard, J. E. Bushnell, S. L. Bernstein and M. T. Bowers J. Phys. Chem. A 106 (42) 10027-10032 (2002) doi: 10.1021/jp021105m.

[234] F. Calvo and E. Yurtsever J. Chem. Phys. 145 (8) 084304 (2016) doi: $10.1063 / 1.496115$.

[235] V. Dryza and E. J. Bieske Int. Rev. Phys. Chem. 32 (4) 559-587 (2013) doi: 10.1080/0144235x.2013.810489.

[236] Artiukhin, D. G., E. J. Bieske and A. A. Buchachenko J. Phys. Chem. A 120 (27) 5006-5015 (2016) doi: 10.1021/acs.jpca.5b12700.

[237] T. D. Della C. H. Suresh Phys. Chem. Chem. Phys. 18 (21) 14588-14602 (2016) doi: 10.1039/c6cp00412a.

[238] M. T. Rodgers and P. B. Armentrout Chem. Rev. 116 (9) 5642-5687 doi: 10.1021/acs.chemrev.5b00688.

[239] E. Coccia, E. Bodo and F. A. Gianturco Europhys. Lett. 8223001 (2008) doi: 10.1209/0295-5075/82/23001.

[240] J. Hernández-Rojas. and D. J. Wales J. Chem. Phys. 119 (15) 7800-7804 (2003) doi: $10.1063 / 1.1608852$.

[241] M. Slama, K. Issa, M. Zbidi and M. B. Rhouma Mol. Phys. 115 (6) 757-770 (2017) 
doi: $10.1080 / 00268976.2017 .1284353$.

[242] D. Prekas, C. Lüder and M. Velegrakis J. Chem. Phys. 108 (11) 4450-4459 (1998) doi: $10.1063 / 1.475856$.

[243] Bruch, L. W., R. D. Diehl and J. A. Venables Rev. Mod. Phys. 79 (4) 1381-1454 (2007) doi: 10.1103/RevModPhys.79.1381.

[244] L. Reatto, D. E. Galli, M. Nava and M. W. Cole J. Phys.-Cond. Matt. 25 (44) 443001 (2013) doi: 10.1088/0953-8984/25/44/443001.

[245] M. C. Gordillo Phys. Rev. B 89 (15) 155401 (2014) doi: 10.1103/PhysRevB.89.155401.

[246] R. Rodríguez-Cantano, R. Pérez de Tudela, M. Bartolomei, M. I. Hernandez, J. Campos-Martínez, T. González-Lezana, P. Villarreal, J. Hernández-Rojas and J. Bretón J. Chem. Phys. 143 (22) 224306 (2015) doi: 10.1063/1.4936414.

[247] M. Bartolomei, R. Pérez de Tudela, K. Arteaga, T. González-Lezana, M. I. Hernández, J. Campos-Martínez, P. Villarreal, J. Hernández-Rojas, J. Bretón and F. Pirani Phys. Chem. Chem. Phys. 19 (38) 26358-26368 (2017) doi: 10.1039/C7CP03819D.

[248] Y. Zhao and D. G. Truhlar J. Phys. Chem. C 112 (11) 4061-4067 (2008) doi: 10.1021/jp710918f.

[249] S. Denifl, F. Zappa, I. Mähr, A. Mauracher, M. Probst, J. Urban, P. Mach, A. Bacher, D. K. Bohme, O. Echt, T. D. Märk and P. Scheier J. Chem. Phys. 132 (23) 234307 (2010) doi: 10.1063/1.3436721.

[250] P. Martini, L. Kranabetter, M. Goulart, M. Kuhn, M. Gatchell, D. K. Bohme and P. Scheier Int. J. Mass Spectr. 434 136-141 (2018) doi: 10.1016/j.ijms.2018.09.020.

[251] M. Gatchell, P. Martini, L. Kranabetter, B. Rasul and P. Scheier Phys. Rev. A 98 (2) 022519 (2018) doi: 10.1103/PhysRevA.98.022519.

[252] S. F. Yang, S. M. Brereton, M. D. Wheeler and A. M. Ellis Phys. Chem. Chem. Phys. 7 (24) 4082-4088 (2005) doi: 10.1039/b511628g.

[253] C. Leidlmair, P. Bartl, H. Schöbel, S. Denifl, T. D. Märk, S. Yang, A. M. Ellis and P. Scheier ChemPhysChem 13 (2) 469-476 (2011) doi: 10.1002/cphc.201100880.

[254] H. Schöbel, C. Leidlmair, P. Bartl, A. Aleem, M. Hager, O. Echt, T. D. Märk and P. Scheier Phys. Chem. Chem. Phys. 13 (3) 1092-1098 (2011). doi: 10.1039/C0CP01268H.

[255] M. Goulart, F. Zappa, A. M. Ellis, P. Bartl, S. Ralser and P. Scheier Phys. Chem. Chem. Phys. 19 (35) 24197-24201 (2017) doi: 10.1039/c7cp02994b.

[256] M. Kuhn, S. Raggl, P. Martini, N. Gitzl, M. M. Darian, M. Goulart, J. Postler, L. Feketeova and P. Scheier Eur. Phys. J. D 72 (2) 38 (2018).

[257] V. K. Shen, D. W. Siderius, W. P. Krekelberg and H. W. Hatch, Eds., NIST Standard Reference Simulation Website, NIST Standard Reference Database Number 173, National Institute of Standards and Technology, Gaithersburg MD, 20899, http://doi.org/ doi: 10.18434/T4M88Q.

[258] S. Yang, S. M. Brereton, S. Nandhra, A. M. Ellis, B. Shang, L.-F. Yuan and J. Yang J. Chem. Phys. 127134303 (2007) doi: 10.1063/1.2772624.

[259] S. Denifl, F. Zappa, I. Mähr, F. Ferreira da Silva, A. Aleem, A. Mauracher, M. Probst, J. Urban, P. Mach, A. Bacher, O. Echt, T. D. Märk and P. Scheier Angew. Chemie 48 (47) 8940-8943 doi: 10.1002/anie.200904381.

[260] O. Echt, A. Kaiser, S. Zöttl, A. Mauracher, S. Denifl and P. Scheier ChemPlusChem 78 (9) 910-920 (2013) doi: 10.1002/cplu.201300198.

[261] M. Mahmoodi-Darian, E. J. A. Maalouf, S. Zöttl, P. Scheier and O. Echt Int. J. Mass Spect. 450116293 (2020) doi: 10.1016/j.ijms.2020.116293.

[262] O. Echt, D. Kreisle, E. Recknagel, J. J. Saenz, R. Casero and J. M. Soler Phys. Rev. A 38 (7) 3236-3248 (1988) doi: 10.1103/PhysRevA.38.3236.

[263] U. Näher S. Bjørnholm, S. Frauendorf, F. Garcias and C. Guet Phys. Rep. 285 (6) 245-320 (1997) doi: 10.1016/S0370-1573(96)00040-3.

[264] O. Echt, P. Scheier and T. D. Märk Compt. Rend. Phys. 3 (3) 353-364 (2002) doi: 10.1016/S1631-0705(02)01325-7.

[265] W. A. Saunders Phys. Rev. Lett. 64 (25) 3046-3049 (1990) doi: 10.1103/PhysRevLett.64.3046.

[266] M. Renzler, M. Harnisch, M. Daxner, L. Kranabetter, M. Kuhn, P. Scheier and O. 
Echt Phys. Chem. Chem. Phys. 18 10623-10629 (2016) doi: 10.1039/C6CP00764C.

[267] M. Mahmoodi-Darian, S. Denifl, M. Probst, S. E. Huber, A. Mauracher, P. Scheier and T. D. Märk Chem. Phys. Lett. 730 527-530 (2019) doi: 10.1016/j.cplett.2019.06.057.

[268] A. Mauracher, M. Daxner, S. E. Huber, J. Postler, M. Renzler, S. Denifl, P. Scheier and A. M. Ellis Angew. Chem. 53 (50) 13794-13797 (2014) doi: 10.1002/anie.201408172.

[269] M. Renzler, M. Daxner, N. Weinberger, S. Denifl, P. Scheier and O. Echt Phys. Chem. Chem. Phys. 16 22466-22470 (2014) doi: 10.1039/C4CP03236E.

[270] A. Mauracher, M. Daxner, S. E. Huber, J. Postler, M. Renzler, S. Denifl, P. Scheier and A. M. Ellis J. Chem. Phys. 142 (10) 104306 (2015) doi: 10.1063/1.4913956.

[271] S. Denifl Eur. Phys. J. 222 (9) 2017-2033 (2013) doi: 10.1140/epjst/e2013-01984-x.

[272] P. W. Stephens and J. G. King, Phys. Rev. Lett. 51, 1538-1541 (1983) doi: 10.1103/PhysRevLett.51.153.

[273] J. Sanderson, H. Tanuma, N. Kobayashi, and Y. Kaneko, J. Phys. B 26 (15), L465L470 (1993) doi: 10.1088/0953-4075/26/15/006.

[274] J. Sanderson, H. Tanuma, N. Kobayashi, and Y. Kaneko, J. Phys. B 27 (14), L433L437 (1994). doi: 10.1088/0953-4075/27/14/021.

[275] K. Hiraoka and T. Mori, J. Chem. Phys. 92 (7), 4408-4416 (1990). doi: $10.1063 / 1.457751$.

[276] D. H. Levy, Ann. Rev. Phys. Chem. 31 (1), 197-225 (1980) doi: 10.1146/annurev.pc.31.100180.001213.

[277] L. F. Dimauro, M. Heaven, and T. A. Miller, Chem. Phys. Lett. 104 (6), 526-532 (1984) doi: 10.1016/0009-2614(84)80022-6.

[278] E. J. Bieske, A. Soliva, M. A. Welker, and J. P. Maier, J. Chem. Phys. 93 (6), 44774478 (1990). doi: 10.1063/1.458732.

[279] E. J. Bieske and J. P. Maier, Chem. Rev. 93 (8), 2603-2621 (1993) doi: 10.1021/cr00024a002.

[280] S. A. Nizkorodov, J. P. Maier, and E. J. Bieske, J. Chem. Phys. 102 (13), 5570-5571 (1995) doi: 10.1063/1.469286.

[281] S. A. Nizkorodov, J. P. Maier, and E. J. Bieske, J. Chem. Phys. 103 (4), 1297-1302 (1995) doi: 10.1063/1.469806.

[282] M. Meuwly, S. A. Nizkorodov, J. P. Maier, and E. J. Bieske, J. Chem. Phys. 104 (11), 3876-3885 (1996) doi: 10.1063/1.471244.

[283] O. Dopfer, D. Roth, and J. P. Maier, Chem. Phys. Lett. 310 (1), 201-208 (1999). doi: 10.1016/S0009-2614(99)00731-9.

[284] E. J. Bieske and O. Dopfer, Chem. Rev. 100 (11), 3963-3998 (2000) doi: $10.1021 / \mathrm{cr} 990064 \mathrm{w}$.

[285] M. Brümmer, C. Kaposta, G. Santambrogio, and K. R. Asmis, J. Chem. Phys. 119 (24), 12700-12703 (2003) doi: 10.1063/1.1634254.

[286] G. A. H. Walker, D. A. Bohlender, J. P. Maier, and E. K. Campbell, Astrophys. J. Lett. 812 (1), L8 (2015) doi: 10.1088/2041-8205/812/1/18.

[287] E. K. Campbell, M. Holz, and J. P. Maier, Astrophys. J. Lett. 826 (1), L4 (2016) doi: $10.3847 / 2041-8205 / 826 / 1 / L 4$.

[288] E. K. Campbell, M. Holz, J. P. Maier, D. Gerlich, G. A. H. Walker, and D. Bohlender, Astrophys. J. 822 (1), 17 (2016) doi: 10.3847/0004-637X/822/1/17.

[289] E. K. Campbell, M. Holz, and J. P. Maier, Astrophys. J. 835 (2), 221 (2017) doi: $10.3847 / 1538-4357 / 835 / 2 / 221$.

[290] F. X. Hardy, C. A. Rice, and J. P. Maier, Astrophys. J. 836 (1), 37 (2017) doi: $10.3847 / 1538-4357 / 836 / 1 / 37$.

[291] D. Gerlich, Phys. Scripta T59, 256-263 (1995) doi: 10.1088/00318949/1995/T59/035.

[292] D. Gerlich, in Low Temperatures and Cold Molecules, edited by I. W. M. Smith (2008), Chap. 6, pp. 295-343.

[293] M. E. Jacox and W. E. Thompson, J. Chem. Phys. 91 (3), 1410-1416 (1989) doi: $10.1063 / 1.457100$.

[294] W. E. Thompson and M. E. Jacox, J. Chem. Phys. 91 (7), 3826-3837 (1989) doi: 
$10.1063 / 1.456868$.

[295] D. Forney, M. Jakobi, and J. P. Maier, J. Chem. Phys. 90 (1), 600-601 (1989) doi: $10.1063 / 1.456461$.

[296] J. Fulara, M. Jakobi, and J. P. Maier, Chem. Phys. Lett. 211 (2), 227 - 234 (1993) doi: 10.1016/0009-2614(93)85190-Y.

[297] B. H. Foing and P. Ehrenfreund, Nature 369, 296 (1994) doi: 10.1038/369296a0.

[298] G. H. Herbig, Ann. Rev. Astron. Astrophys. 33 (1), 19-73 (1995) doi: 10.1146/annurev.aa.33.090195.000315.

[299] P. J. Sarre, J. Mol. Spect. 238 (1), 1-10 (2006) doi: 10.1016/j.jms.2006.03.009.

[300] S. Smolarek, N. B. Brauer, W. J. Buma, M. Drabbels, J. Am. Chem. Soc. 132 (40), 14086-14091 (2010) doi: 10.1021/ja1034655

[301] N. B. Brauer, S. Smolarek, X. Zhang, W. J. Buma, M. Drabbels, J. Phys. Chem. Lett. 2 (13), 1563-1566 (2011) doi: 10.1021/jz200632s

[302] X. Zhang, N. B. Brauer, G. Berden, A. M. Rijs, M. Drabbels, J. Chem. Phys. 136 (4), 044305 (2012) doi: 10.1063/1.3678011

[303] X. Zhang, M. Drabbels, J. Phys. Chem. Lett. 5 (18), 3100-3105 (2014) doi: $10.1021 / \mathrm{jz} 501530 \mathrm{e}$

[304] M. Okumura, L. I. Yeh, and Y. T. Lee, J. Chem. Phys. 83 (7), 3705-3706 (1985) doi: 10.1063/1.449127.

[305] H. J. Zeng, N. Yang, and M. A. Johnson, Faraday Discuss. 217, 8-33 (2019) doi: 10.1039/C9FD00030E.

[306] A. Kaiser, J. Postler, M. Ončák, M. Kuhn, M. Renzler, S. Spieler, M. Simpson, M. Gatchell, M. K. Beyer, R. Wester, F. A. Gianturco, P. Scheier, et al., J. Phys. Chem. Lett. 9 (6), 1237-1242 (2018) doi: 10.1021/acs.jpclett.8b00150.

[307] A. G. G. M. Tielens, Ann. Rev. Astron. Astrophys. 46 (1), 289-337 (2008) doi: 10.1146/annurev.astro.46.060407.145211.

[308] M. Ben-Nun and T. J. Martínez, Adv. Chem. Phys. 121, 439 (2002) doi: 10.1002/0471264318.ch7.

[309] R. Zhang, B. Lev, J. E. Cuervo, S. Y. Noskov, and D. R. Salahuba, Adv. Quant. Chem. 59, 353 (2010) doi: 10.1016/S0065-3276(10)59010-5.

[310] U. Senff and P. Burton, Mol. Phys. 58 (3), 637-645 (1986) doi: 10.1080/00268978600101441.

[311] A. W. K. Leung, R. R. Julian, and W. H. Breckenridge, J. Chem. Phys. 110 (17), 8443-8447 (1999) doi: 10.1063/1.478805.

[312] A. W. K. Leung, R. R. Julian, and W. H. Breckenridge, J. Chem. Phys. 111 (11), 4999-5003 (1999) doi: 10.1063/1.479737.

[313] P. Soldán, E. P. F. Lee, and T. G.Wright, Mol. Phys. 97 (1-2), 139-149 (1999) doi: 10.1080/00268979909482816.

[314] A. M. Sapse, A. Dumitra, and D. C. Jain, J. Clust. Sci. 14 (1), 21-30 (2003) doi: 10.1023/A:1022997323484.

[315] T. Nagata, M. Aoyagi, and S. Iwata, J. Phys. Chem. A 108 (4), 683-690 (2004) doi: 10.1021/jp036443h.

[316] K. T. Giju, S. Roszak, R. W. Gora, and J. Leszczynski, Chem. Phys. Lett. 391 (1), 112 - 119 (2004) doi: 10.1016/j.cplett.2004.04.08.

[317] F. Sebastianelli, E. Bodo, I. Baccarelli, C. Di Paola, F. A. Gianturco, and M. Yurtsever, Comp. Mat. Sci. 35, 261-267 (2006) doi: 10.1016/j.commatsci.2004.07.011.

[318] J. V. Pototschnig, F. Lackner, A. W. Hauser, and W. E. Ernst, Phys. Chem. Chem. Phys. 19, 14718 (2017) doi: 10.1039/c7cp02332d.

[319] D. Bellert and W. H. Breckenridge, Chem. Rev. 102, 1595 (2002) doi: $10.1021 / \mathrm{cr} 980090 \mathrm{e}$.

[320] A. J. Stone, in International Series of Monographs on Chemistry (1996).

[321] I. G. Kaplan, Intermolecular Interactions: Physical Picture, Computational Methods and Model Potentials (2006).

[322] R. Ahlrichs, R. Penco, and G. Scoles, Chem. Phys. 19 (2), 119 - 130 (1977) doi: 10.1016/0301-0104(77)85124-0.

[323] J. Hepburn, G. Scoles, and R. Penco, Chem. Phys. Lett. 36 (4), 451 - 456 (1975) doi: 
10.1016/0009-2614(75)80278-8.

[324] K. T. Tang and J. P. Toennies, J. Chem. Phys. 80 (8), 3726-3741 (1984) doi: 10.1063/1.447150.

[325] S. H. Patil, J. Chem. Phys. 86 (12), 7000-7006 (1987) doi: 10.1063/1.452348.

[326] R. Ahlrichs, H. J. Böhm, S. Brode, K. T. Tang, and J. P. Toennies, J. Chem. Phys. 88 (10), 6290-6302 (1988) doi: 10.1063/1.454467.

[327] R. A. Aziz and M. J. Slaman, J. Chem. Phys. 94 (12), 8047-8053 (1991) doi: $10.1063 / 1.460139$.

[328] K. T. Tang and J. P. Toennies, J. Chem. Phys. 118 (11), 4976-4983 (2003) doi: 10.1063/1.1543944.

[329] F. Pirani, S. Brizi, L. Roncaratti, P. Casavecchia, D. Cappelletti, and F. Vecchiocattivi, Phys. Chem. Chem. Phys 10, 5489-5503 (2008) doi: 10.1039/B808524B.

[330] A. Andrejeva, W. H. Breckenridge, and T. G. Wright, J. Phys. Chem. A 119 (44), 10959-10970 (2015) doi: 10.1021/acs.jpca.5b08045.

[331] A. M. Reilly and A. Tkatchenko, Chem. Sci. 6, 3289-3301 (2015) doi: 10.1039/C5SC00410A.

[332] I. Yourshaw, Y. Zhao, and D. M. Neumark, J. Chem. Phys. 105 (2), 351-373 (1996) doi: $10.1063 / 1.471893$.

[333] M. Ben El Hadj Rhouma, F. Calvo, and F. Spiegelman, J. Phys. Chem. A 110 (15), 5010-5016 (2006) doi: 10.1021/jp060153u.

[334] A. G. Donchev, Phys. Rev. A 76, 042713 (2007) doi: 10.1103/PhysRevA.76.042713.

[335] A. Andrejeva, A. M. Gardner, J. B. Graneek, R. J. Plowright, W. H. Breckenridge, and T. G. Wright, J. Phys. Chem. A 117 (50), 13578-13590 (2013) doi: $10.1021 /$ jp4075652.

[336] F. Marinetti, E. Coccia, F. A. Gianturco, E. Yurtsever, M. Yurtsever, and E. Yildirim, Theor. Chem. Acc. 118, 53 (2007) doi: 10.1007/s00214-006-0240-z.

[337] M. Bartolomei, F. Pirani and J. Marques Phys. Chem. Chem. Phys. 21 (2) 1600516016 (2019) doi: 10.1039/C9CP02658D

[338] F. Pirani, M. Alberti, A. Castro, M. M. Teixidor and D. Cappelletti, Chem. Phys. Lett. 394 (1) 37 (2004) doi: 10.1016/j.cplett.2004.06.100.

[339] M. Bartolomei, E. Carmona-Novillo, M. I. Hernández, J. Campos-Martínez and F. Pirani J. Phys. Chem. C 11710512 (2) (2013) doi: 10.1021/jp401635t.

[340] M. Buzzacchi, D. E. Galli, and L. Reatto, Phys. Rev. B 64, 094512 (2001) doi: 10.1103/PhysRevB.64.094512.

[341] K. K. Lehmann, Phys. Rev. Lett. 88, 145301 (2002) doi: 10.1103/PhysRevLett.88.145301.

[342] E. Bodo, E. Coccia, D. López-Durán, and F. A. Gianturco, Phys. Scr. 76, C104 (2013) doi: 10.1088/0031-8949/76/3/N16.

[343] E. Yurtsever, E. Yildirim, M. Yirtsever, E. Bodo, and F. A. Gianturco, Eur. Phys. J. D 43, 105-108 (2007) doi: 10.1140/epjd/e2007-00068-x.

[344] M. Mella and F. Cargnoni, J. Phys. Chem. A 118 (33), 6473-6483 (2014) doi: $10.1021 / j p 5027306$.

[345] A. Golov and S. Sekatskii, Z. Phys. D 27 (4), 349-355 (1993) doi: 10.1007/BF01437468.

[346] F. Stienkemeier and K. K. Lehmann, J. Phys. B: Atom. Mol. Opt. Phys. 39 (8), R127 (2006) doi: 10.1088/0953-4075/39/8/R01.

[347] F. Ancilotto, M. Pi, R. Mayol, M. Barranco, and K. K. Lehmann, J. Phys. Chem. A 111 (49), 12695-12701 (2007) doi: 10.1021/jp076069b.

[348] D. Mateo, A. Leal, A. Hernando, M. Barranco, M. Pi, F. Cargnoni, M. Mella, X. Zhang, and M. Drabbels, J. Chem. Phys. 140 (13), 131101 (2014) doi: $10.1063 / 1.4870245$.

[349] S. L. Fiedler, D. Mateo, T. Aleksanyan, and J. Eloranta, Phys. Rev. B 86, 144522 (2012) doi: 10.1103/PhysRevB.86.144522.

[350] F. Calvo, in Clusters: Structure, Bonding and Reactivity, edited by M. T. Nguyen and B. Kiran (Springer International Publishing, Cham, 2017), pp. 137-156.

[351] M. Bartolomei, M. I. Hernández, J. Campos-Martínez and R. Hernández-Lamoneda, 
International Reviews in Physical Chemistry
Carbon 144, $724-730$ (2019) doi: $10.1016 /$ j.carbon.2018.12.086.

Carbon 144, $724-730$ (2019) doi: $10.1016 /$ j.carbon. 2018.12 .086$. 